\title{
The Complete Matter Sector in a Three-Generation Compactification
}

\author{
Per Berglund ${ }^{1}$, Tristan Hübsch ${ }^{2 \star}$ and Linda Parkes ${ }^{1}$ \\ 1 Theory Group, Department of Physics, University of Texas, Austin, TX 78712, USA \\ BERGLUND, PARKES@UTAPHY.BITNET \\ ${ }^{2}$ Harvard University, Departments of Mathematics and Physics, Cambridge, MA 02138, USA \\ HUBSCH@ZARISHI.HARVARD.EDU HUBSCH@HUHEPL.BITNET
}

Received March 21, 1991

\begin{abstract}
We consider a Calabi-Yau compactification paradigm with three light generations and an $R$-symmetry. From a special form of the Tian-Yau manifold, we also construct a new three-generation model with markedly different phenomenology. The complete spectrum of all light matter fields is obtained in a universal way and moreover in a physically suitable basis, allowing a straightforward analysis of all their couplings. Here we discuss all the renormalizable Yukawa couplings. This computation can equally well be repeated for all compactification models based on Calabi-Yau complete intersections in products of homogeneous spaces.
\end{abstract}

\section{Introduction}

The main purpose of this article is to provide a comprehensive presentation of the cohomology techniques of exact and spectral sequences (TESS for short) [1-4] with the aid of which the complete light matter sector for a large class of CalabiYau string compactifications can be computed. In principle, this includes all the couplings and here we exemplify this by discussing the renormalizable terms; more general results and especially non-renormalizable couplings are reported in [5].

In this article, we focus on a construction that features a discrete $R$-symmetry. The reason for this is twofold. Firstly, it has been argued recently [6] that the $R$-symmetry in this model ensures the existence of a flat direction in the field space which allows a deformation of the model in which the initial $E_{6}$ gauge-symmetry is broken to $S O(10)$ or even $S U(5)$. Here we show that, upon inclusion of all light matter superfields, this deformation indeed is possible; a more complete analysis will be given in ref. [5]. In addition, we find two distinct three-generation models obtained following the Tian-Yau construction [7], one of which exhibits several phenomenologically interesting effects. They differ in the discrete symmetries of the interactions among the matter superfields.

* On leave of absence from the "Ruder Bošković" Institute, Zagreb, Croatia 
Our technique, TESS, is universally valid for any complex compact manifold $\mathscr{M}$ (Calabi-Yau or not) embedded in a product of so-called flag-spaces

$$
\mathbb{F}_{n_{1}, \ldots, n_{f}} \approx\left\{\frac{U(N)}{U\left(n_{1}\right) \times \cdots \times U\left(n_{f}\right)}\right\},
$$

by means of a system of homogeneous holomorphic polynomial constraints. For immediate physics application and to avoid challenging the readers' patience with overly complicated notation, we discuss a particular Calabi-Yau manifold constructed as the space of solutions to a system of three constraints (2.2) in $\mathbb{P}_{x}^{3} \times \mathbb{P}_{y}^{3}$ [7]. Members of this deformation class have been used to construct possibly realistic string compactification models [8-10] and our present study is also meant to provide further physically interesting information.

All heterotic superstring models in 3 + 1-dimensional spacetime with an "internal" Calabi-Yau space $\mathscr{M}$ contain chiral superfields which are massless at the compactification scale $M_{C}$. They come in 1-1 correspondence with certain harmonic forms on $\mathscr{M}$. In addition to the moduli superfields and the universally present superfield containing the dilaton and the axion, compactification gives rise to chiral superfields (and clearly their antichiral CPT conjugates also) which stem from the $E_{8}$ Yang-Mills supermultiplet and which we collectively label as "matter." Up to $E_{6}$ symmetry breaking effects, the matter superfields occur in $27 \mathrm{~s}, 27$ s and 1s of $E_{6}$, corresponding respectively to $\mathscr{T}_{\mathcal{M}^{-}}, \mathscr{T}_{\mathscr{M}}^{*}$-, and End $\mathscr{T}_{\mathcal{M}^{-}}$-valued (Dolbeault) 1 -forms on $\mathscr{M}$.

The matter superfields and their couplings have been analyzed in much detail for several particular models, but only in part. Often, and certainly in the present case, the 27s can be represented faithfully by the popular technique of polynomial deformations, with which the $\left(27^{3}\right)$ couplings [11] can also be computed. TESS consistently generalizes this [3].

For the Tian-Yau manifold [7], the $27 *$ s can be parametrized and the $(27 * 3)$ Yukawa couplings computed using the geometry of certain exceptional lines [12]. This is also true of some 45 similar examples [13] and several more models scattered in the literature. Often much can be learned merely from symmetry considerations, for which a cunning use of the "fixed point" and "hyperplane" theorems of Lefschetz will do the trick [9]. TESS is in complete agreement with these special techniques but is much more widely applicable.

Until recently, the 1s have eluded analysis and only a lower bound on their multiplicity was known [14]. In describing them, polynomial deformations are notoriously incomplete $[15,16,17]$ and the Lefschetz theorems are of no use since End $\mathscr{T}_{\mathscr{M}}$ is a self-dual bundle. Again, TESS is effective but, for complete results, we have to go beyond the description in ref. $[3,4,15]$ and, as in ref. [16, 17], employ the identification

$$
\mathbb{P}^{n}=\frac{U(n+1)}{U(1) \times U(n)}
$$

and also make extensive use of the $U(n)$ tensor algebra. ${ }^{1}$

The main concrete result of this article is the parametrization of all $27 \mathrm{~s}, 27$ s and $1 \mathrm{~s}-$ on the same footing and moreover in a physically suitable basis. Hence, all

\footnotetext{
1 This ingenious technique is remembered as "advanced magic" in Cambridge, England, where it was conceived by Michael Eastwood [2]; we are grateful to the Master for teaching us
} 
the $\left(27^{3}\right),\left(27^{* 3}\right),\left(1^{3}\right)$ and $(27 \cdot 27 * \cdot 1)$ Yukawa and also many non-perturbative couplings can be analyzed easily. We determine many of these merely by using symmetries and use this to check our generalization of the Yukawa coupling computation of ref. [11]; the complete determination of all couplings requires further study however [5].

Before we proceed, a remark is in order. Ultimately, all of the matter superfields are supposed to acquire realistic masses and this is clearly a model dependent issue. However, many of the $1 \mathrm{~s}$ are expected to become massive in a model-independent way, through the world sheet instanton effects [18] whence their mass is schematically

$$
m \sim M_{C} \exp \left\{-M_{\mathrm{Pl}} / M_{C}\right\} .
$$

One would expect the compactification mass-scale $M_{C}$ to be roughly of the order of the Planck mass and the 1s would appear phenomenologically irrelevant. However, due to the exponential, $m$ is very sensitive to the ratio $M_{\mathrm{Pl}} / M_{C}$; even a small gap of just one or two orders of magnitude between $M_{\mathrm{P} 1}$ and $M_{C}$ (which is expected anyway, for several different reasons) can bring $m$ into a phenomenologically interesting range. Since the Yang-Mills charged matter superfield 27-27* pairs couple directly to the $1 \mathrm{~s}$, the latter must not be ignored. Moreover, they may have a desirable phenomenological impact $[19,20]$.

Understanding that spectral sequences appear novel, hence possibly intimidating and to avoid cluttering the main part of the article, we have deferred most of the technical details and some instructive sample computations to the appendices. A review of the basic notation, the definition of the particular Calabi-Yau manifold considered here and an introduction to some of the basics of TESS is however collected in Sect. 2. The skilled reader will no doubt skip this section but the majority will, we hope, find it useful.

In Sect. 3, we use TESS to parametrize the 27 and the $27^{*}$ matter superfields while the 1s are parametrized in Sect. 4. A brief discussion of the two distinct three-generation models spans Sect. 5. For future reference, we also discuss the restrictions which the $R$-symmetry puts on the Yukawa couplings.

In Sect. 6, we relate the TESS parametrization of $27 \mathrm{~s}$ to the polynomial deformations of Ref. [11] (see also ref. $[9,21]$ ) and discuss the computation of the $\left(27^{3}\right)$ Yukawa couplings. In Sect. 7, we use symmetries to determine most of the $\left(27^{* 3}\right)$ Yukawa couplings and then relate the TESS parametrization of $27 *$ s to the exceptional lines used in ref. $[12,13]$. Using this correspondence, we evaluate all these Yukawa couplings, providing that a suitable generalization of the $\left(27^{3}\right)$ coupling formula [11] applies to $\left(27^{* 3}\right)$ too.

In Sect. 8 , we use the advantage of the TESS parametrization: we can use discrete symmetries to determine many of the $\left(1^{3}\right)$ and the $\left(27 \cdot 27^{*} \cdot 1\right)$ couplings. We also note the relation to the computation of ref. [11], providing a universally applicable generalization. Section 9 contains our concluding remarks and discussion.

\section{The Manifold and the Technique}

The family of Tian-Yau manifolds [7] is constructed as a family of embeddings in

$$
\mathscr{W} \stackrel{\text { def }}{=} \mathbb{P}_{x}^{3} \times \mathbb{P}_{y}^{3}, \quad \mathbb{P}^{3}=\left\{\frac{U(4)}{U(1) \times U(3)}\right\},
$$


by means of a system of three holomorphic homogeneous equations. In this article, we discuss the particular choice

$$
\begin{aligned}
f(x) & =\left(x^{1}\right)^{3}+\left(x^{2}\right)^{3}+\left(x^{3}\right)^{3}+\left(x^{4}\right)^{3}=0, \\
g(x) & =\left(y^{1}\right)^{3}+\left(y^{2}\right)^{3}+\left(y^{3}\right)^{3}+\left(y^{4}\right)^{3}=0, \\
h(x, y) & =x^{1} y^{1}+x^{2} y^{2}+x^{3} y^{3}=0 .
\end{aligned}
$$

By $x^{i}\left(y^{i}\right)$ we denote the homogeneous coordinates of the first (second) $\mathbb{P}^{3}$ factor. By Proposition 2 of [22], the choice (2.2) yields a smooth manifold; for the sake of completeness, we include the proof in Appendix A. Interestingly, this choice appears to have been omitted in the classification of ref. [23]; in fact it possesses symmetries that none of the models in that classification do.

Our objective is to find suitable representatives of the matter $27 \mathrm{~s}, 27$ *s and $1 \mathrm{~s}$, i.e., the corresponding elements of $H^{1}\left(\mathscr{M}, \mathscr{T}_{\mathscr{M}}\right), H^{1}\left(\mathscr{M}, \mathscr{T}_{\mathscr{M}}^{*}\right)$ and $H^{1}\left(\mathscr{M}\right.$, End $\left.\mathscr{T}_{\mathscr{M}}\right)$. We will express these in terms of readily available geometrical data on $\mathscr{W}$. To this end, we have to (1) relate the bundles $\mathscr{T}_{\mathscr{M}}, \mathscr{T}_{\mathscr{M}}^{*}$ and End $\mathscr{T}_{\mathscr{M}}$ to bundles over $\mathscr{W},(2)$ determine the required cohomology on $\mathscr{M}$ in terms of that on $\mathscr{W}$ and (3) find all required cohomology on $\mathscr{W}$. The actual computation then proceeds in reverse order.

2.1. $\mathscr{T}_{\mathscr{M}}$ from Bundles over $\mathscr{W}$. The first of these tasks is accomplished by using the short exact sequence

$$
\left.\left.0 \longrightarrow \mathscr{T}_{\mathscr{M}} \stackrel{i}{\longrightarrow} \mathscr{T}_{\mathscr{W}}\right|_{\mathscr{M}} \stackrel{j}{\longrightarrow} \mathscr{E}\right|_{\mathscr{M}} \longrightarrow 0
$$

where

$$
\mathscr{E} \stackrel{\text { def }}{=} \mathscr{E}_{f} \oplus \mathscr{E}_{g} \oplus \mathscr{E}_{h}
$$

Each hypersurface in the bundle $\mathscr{E}_{f}($ over $\mathscr{W}$ ) is defined by a homogeneous polynomial of the degree of $f$, while $\mathscr{E}_{g}$ and $\mathscr{E}_{h}$ correspond similarly to polynomials of the degree of $g$ and $h$, respectively. $\left.\right|_{\mathscr{M}}$ denotes the restriction to $\mathscr{M}$. We will also use the dual of (2.3),

$$
\left.\left.0 \longrightarrow \mathscr{E}^{*}\right|_{\mathscr{M}} \stackrel{j}{\longrightarrow} \mathscr{T}_{\mathscr{W}}^{*}\right|_{\mathscr{M}} \stackrel{i}{\longrightarrow} \mathscr{T}_{\mathscr{M}}^{*} \longrightarrow 0
$$

and sequences obtained by tensoring either of these with various bundles over $\mathscr{W}$.

In general, a sequence of maps and spaces is exact if the kernel ${ }^{2}$ of every map is the image of the preceding one. Indeed, $i$ embeds $\mathscr{T}_{\mathcal{M}}$ in $\mathscr{T}_{\mathscr{W}}$ and annihilates only $0 \in \mathscr{T}_{\mathcal{M}}$ (to preserve the additive group structure of tangent vectors). $j$ is the projection transversal to $\mathscr{M}$ and annihilates all vectors in $\left.\mathscr{T}_{\mathscr{W}}\right|_{\mathscr{M}}$ which are tangential to $\mathscr{M}$ - precisely the image of $i$. Being a projection, $j$ covers all of $\left.\mathscr{E}\right|_{\mathscr{M}}$ - precisely what the right-most map annihilates. The exactness of the sequence (2.3) is equivalent to the assertion that $\mathscr{E}_{\mathscr{M}}=\left\{\left.\mathscr{T}_{\mathscr{W}}\right|_{\mathscr{M}} \mid \mathscr{T}_{\mathcal{M}}\right\}$. Note: $\left.\mathscr{T}_{\mathscr{W}}\right|_{\mathscr{M}}$ is the direct sum of $\mathscr{T}_{\mathcal{M}}$ and $\left.\mathscr{E}\right|_{\mathscr{M}}$ only differentiably, not holomorphically; any metric in which $\mathscr{T}_{\mathcal{M}}$ and $\left.\mathscr{E}\right|_{\mathscr{M}}$ are orthogonal invariably fails to be hermitian.

${ }^{2}$ The kernel of a map consists of all the elements which are annihilated by it 
It is a basic fact (p. 40 of Griffiths and Harris in ref. [1]) that a short exact sequence such as (2.3) induces an accompanying long exact cohomology sequence

$$
\begin{aligned}
& 0 \longrightarrow H^{0}\left(\mathscr{M}, \mathscr{T}_{\mathscr{M}}\right) \stackrel{i}{\longrightarrow} H^{0}\left(\mathscr{M}, \mathscr{T}_{\mathscr{W}}\right) \stackrel{j}{\longrightarrow} H^{0}(\mathscr{M}, \mathscr{E}) \\
& \stackrel{\delta_{0}}{\longrightarrow} H^{1}\left(\mathscr{M}, \mathscr{T}_{M}\right) \stackrel{i}{\longrightarrow} H^{1}\left(\mathscr{M}, \mathscr{T}_{\mathscr{W}}\right) \stackrel{j}{\longrightarrow} H^{1}(\mathscr{M}, \mathscr{E}) \\
& \stackrel{\delta_{1}}{\longrightarrow} \ldots
\end{aligned}
$$

and this is what we shall be using throughout.

2.2. Harmonic Forms on $\mathscr{M}$ from those on $\mathscr{W}$. The second task is to obtain forms on $\mathscr{M}$ from the cohomology on $\mathscr{W}$. Let $\mathcal{O}_{\mathscr{W}}$ and $\mathcal{O}_{\mathscr{M}}$ denote the (sheaves of) holomorphic functions (scalars) on $\mathscr{W}$ and $\mathscr{M}$, respectively. The restriction $\left.\mathcal{O}_{\mathscr{W}} \stackrel{\varrho}{\rightarrow} \mathcal{O}_{\mathscr{W}}\right|_{\mathcal{M}}=\mathcal{O}_{\mathcal{M}}$ will prove to be of key importance.

$\mathscr{E}_{f}$ is a line bundle over $\mathbb{P}_{x}^{3}$. This means that to every point $x \in \mathbb{P}_{x}^{3}$, it associates a $\mathbb{C}^{1}$-like vector space, which varies holomorphically as $x$ sweeps through $\mathbb{P}_{x}^{3}$. Now, $\mathscr{E}_{f}$ is chosen to correspond to polynomials $\varphi_{(a b c)} x^{a} x^{b} x^{c}$, of the same type as $f(x)$. Then, at any particular point in $\mathbb{P}_{x}^{3}$, the fibre of $\mathscr{E}_{f}$ is generated by the $(\mathbb{C}$-number) value of such cubics, each of which simply corresponds to another point in $\mathbb{C}^{1}$-like fibre. The number of inequivalent cubics $\varphi_{(a b c)} x^{a} x^{b} x^{c}$ (considering now the $x^{a}$ as global coordinates over $\mathbb{P}_{x}^{3}$ ) is a related but different issue, telling the dimension of the space of global holomorphic sections of $\mathscr{E}_{f}$. Because of this relation, one says that the line bundle $\mathscr{E}_{f}$ is generated by its sections and is hence represented by totally symmetric covariant tensors such as $\varphi_{(a b c)}$.

The dual bundle, $\mathscr{E}_{f}^{*}$, is then formally represented by contravariant tensors, $\phi^{(a b c)}$, such that we have the natural contraction (scalar product) $\langle\phi \mid \varphi\rangle \stackrel{\text { def }}{=} \phi^{(a b c)} \varphi_{(a b c)} \in \mathcal{O}_{\mathscr{W}}$. Let $\phi^{(a b c)}, \gamma^{(\alpha \beta \gamma)}$ and $\eta^{a \alpha}$ be general elements of $\mathscr{E}_{f}^{*}$, $\mathscr{E}_{g}^{*}$, and $\mathscr{E}_{h}^{*}$, respectively.

Contracting $\mathscr{E}^{*}$ with the triple $\xi=\left(f_{\star}, g_{\star}, h_{\star}\right),{ }^{3}$ we obtain, for $\lambda, \mu, v \in \mathbb{C}$,

$$
\left(f_{\star}, g_{\star}, h_{\star}\right) \cdot\left(\lambda \phi^{\star}, \mu \gamma^{\star}, v \eta^{\star}\right)=\lambda\langle\phi \mid f\rangle+\mu\langle\gamma \mid g\rangle+v\langle\eta \mid h\rangle \in \mathcal{O}_{\mathscr{W}} .
$$

Thus, in the sequence

$$
\mathscr{E} * \stackrel{\xi}{\longrightarrow} \mathcal{O}_{\mathscr{W}} \stackrel{\varrho}{\longrightarrow} \mathcal{O}_{\mathscr{M}} \longrightarrow 0
$$

the map provided by contraction with $\xi=\left(f_{\star}, g_{\star}, h_{\star}\right)$ covers all of $\mathcal{O}_{\mathscr{W}}$ where $\xi \neq 0$, i.e., at all points of $(\mathscr{W}-\mathscr{M})$. Therefore, $\varrho$ vanishes there and so does $\mathcal{O}_{\mathscr{M}}$. At $\mathscr{M} \subset \mathscr{W}$ however, the triple of polynomials $\xi$ vanishes and the map $\xi$ is null, whence $\varrho$ identifies $\mathcal{O}_{\mathscr{M}}$ with $\left.\mathcal{O}_{\mathscr{W}}\right|_{\mathscr{M}}$, the restriction of $\mathcal{O}_{\mathscr{W}}$ to $\mathscr{M}$.

To complete this sequence to the left, note that the map $\xi$ has a kernel. In fact, all complex linear combinations of

$$
\left(-\langle\gamma \mid g\rangle \phi^{\star},\langle\phi \mid f\rangle \gamma^{\star}, 0\right),\left(\langle\eta \mid h\rangle \phi^{\star}, 0,-\langle\phi \mid f\rangle \eta^{\star}\right),\left(0,-\langle\eta \mid h\rangle \gamma^{\star},\langle\gamma \mid g\rangle \eta^{\star}\right),
$$

${ }^{3}$ In this section, we use the star " $\star$ " in the sub- or superscript merely to remind the reader of the position of the indices; it must not be confused with the symbol *, which denotes the dual 
in $\mathscr{E}^{*}$, are annihilated by contraction with $\xi$; such triples $\operatorname{span} \operatorname{ker}\left(\mathscr{E}^{*} \stackrel{\xi}{\rightarrow} \mathcal{O}_{\mathscr{W}}\right)$. The very same elements of $\mathscr{E}^{*}$ are also obtained from

$$
\wedge^{2} \mathscr{E}^{*}=\mathscr{E}_{f}^{*} \wedge \mathscr{E}_{g}^{*} \oplus \mathscr{E}_{f}^{*} \wedge \mathscr{E}_{h}^{*} \oplus \mathscr{E}_{g}^{*} \wedge \mathscr{E}_{h}^{*}
$$

by mapping through $\xi$. To see this, note that $\wedge^{2} \mathscr{E}^{*}$ is represented by linear combinations of

$$
\left(\begin{array}{ccc}
0 & \phi^{\star} \gamma^{\star} & 0 \\
-\phi^{\star} \gamma^{\star} & 0 & 0 \\
0 & 0 & 0
\end{array}\right),\left(\begin{array}{ccc}
0 & 0 & -\phi^{\star} \eta^{\star} \\
0 & 0 & 0 \\
\phi^{\star} \eta^{\star} & 0 & 0
\end{array}\right),\left(\begin{array}{ccc}
0 & 0 & 0 \\
0 & 0 & \gamma^{\star} \eta^{\star} \\
0 & -\gamma^{\star} \eta^{\star} & 0
\end{array}\right)
$$

(the antisymmetry of the wedge product, $\wedge$, accounts for the alternating signs). Then, for example,

$$
\left(f_{\star}, g_{\star}, h_{\star}\right) \cdot\left(\begin{array}{ccc}
0 & \phi^{\star} \gamma^{\star} & 0 \\
-\phi^{\star} \gamma^{\star} & 0 & 0 \\
0 & 0 & 0
\end{array}\right)=\left(-\langle\gamma \mid g\rangle \phi^{\star},\langle\phi \mid f\rangle \gamma^{\star}, 0\right) .
$$

Iterating this procedure, we obtain the Koszul complex, represented by the (sheaf-) exact sequence

$$
0 \longrightarrow \wedge^{3} \mathscr{E} * \stackrel{\xi}{\longrightarrow} \wedge^{2} \mathscr{E} * \stackrel{\xi}{\longrightarrow} \mathscr{E} * \stackrel{\xi}{\longrightarrow} \mathcal{O}_{\mathscr{W}} \stackrel{\varrho}{\longrightarrow} \mathcal{O}_{\mathscr{M}} \longrightarrow 0
$$

This sequence relates holomorphic functions (scalars) on $\mathscr{M}\left(\mathcal{O}_{\mathscr{M}}\right)$ to quantities defined entirely on $\mathscr{W}$; the analogous will also be true if we tensor the whole sequence with a vector bundle over $\mathscr{W}$, such as $\mathscr{T}_{\mathscr{W}}$ or $\mathscr{E}$.

However, unlike (2.3) which accompanied by (2.6), (2.10) is not accompanied by an exact cohomology sequence. The derived cohomology groups can again be arranged in analogy to (2.6)

\begin{tabular}{cccc|c}
$\wedge^{3} \mathscr{E}^{*}$ & $\wedge^{2} \mathscr{E}^{*}$ & $\mathscr{E}^{*}$ & $\mathcal{O}_{\mathscr{W}}$ & $\mathcal{O}_{\mathscr{M}}$ \\
\hline$H^{0}\left(\mathscr{W}, \wedge^{3} \mathscr{E}^{*}\right)$ & $H^{0}\left(\mathscr{W}, \wedge^{2} \mathscr{E} *\right)$ & $H^{0}\left(\mathscr{W}, \mathscr{E}^{*}\right)$ & $H^{0}(\mathscr{W})$ & $H^{0}(\mathscr{M})$ \\
$H^{1}\left(\mathscr{W}, \wedge^{3} \mathscr{E}^{*}\right)$ & $H^{1}\left(\mathscr{W}, \wedge^{2} \mathscr{E} *\right)$ & $H^{1}\left(\mathscr{W}, \mathscr{E}^{*}\right)$ & $H^{1}(\mathscr{W})$ & $H^{1}(\mathscr{M})$ \\
$\vdots$ & $\vdots$ & $\vdots$ & $\vdots$ & $\vdots$
\end{tabular}

but there is no analogue of $\delta_{q}$. Instead, there will now be maps ("differentials" $d_{i}$ ) that act $i+1$ steps to the right and $i$ steps up the chart. The "horizontal" maps, such as in (2.6), are the $i=0$ case thereof. From the action of these maps, the cohomology in the right-most column is determined in terms of the cohomology groups which appear in the lower left quadrant of (2.11).

In practice, one starts from (2.11), called the $0^{\text {th }}$ level of the spectral sequence. The action of the differentials $d_{i}$ is then found, order by order in $i$, and one passes to 
cohomology quotients

$$
\left\{\left\{\omega: d_{i} \omega=0\right\} /\left\{\omega^{\prime}=d_{i} \alpha\right\}\right\}
$$

in the lower left quadrant of (2.11). It should be obvious that this sequence of approximations converges for some $i \leqq 3$; when $i>3$, even for the entries in the left-most column, the maps would point out of the lower left quadrant of (2.11).

Now, contributions to $H^{q}(\mathscr{M})$ consist only of $d_{i}$-closed (modulo $d_{i}$-exact) forms, for all $i$. In practice, we advance order by order in $i$ cancelling out all pairs $d_{i} \alpha=\beta$. What remains, abuts to $H^{q}(\mathscr{M})$ according to

$$
\left\{H^{q+k}\left(\mathscr{W}, \wedge^{k} \mathscr{E} *\right), \forall k: “ d_{i} \text {-closed } / d_{i} \text {-exact”, } \forall i\right\} \Rightarrow H^{q}(\mathscr{M})
$$

Appendix $\mathrm{C}$ provides more details. The collection of such contributions does not form a direct sum in $H^{q}(\mathscr{M})$ but this will be of little consequence for our purposes; nevertheless, following ref. [2], we shall use the ' + ' symbol instead of ' $\oplus$ '.

The astute reader will have noticed that computations employing the long exact cohomology sequences (2.6), and even more so the spectral sequences (2.11), rest upon the ability to discern the action of several maps. This otherwise hopeless taks is however straightforwardly accomplished using the coset representation of $\mathbb{P}^{3}$ (2.1) and $U(4)$ and $U(3)$ tensor algebra. (More details can be found in Appendix B, ref. [16] and ref. [2].)

2.3. A Quick Example. Before we engage in the computations of $H^{1}\left(\mathscr{M}, \mathscr{T}_{\mathscr{M}}\right)$, $H^{1}\left(\mathscr{M}, \mathscr{T}_{\mathscr{M}}^{*}\right)$ and $H^{1}\left(\mathscr{M}\right.$, End $\left.\mathscr{T}_{\mathscr{M}}\right)$, let us quickly examine the spectral sequence accompanying the "bare" Koszul complex (2.10).

To begin with, we rewrite $(2.10)$ in the Young tableau notation described in Appendix B:

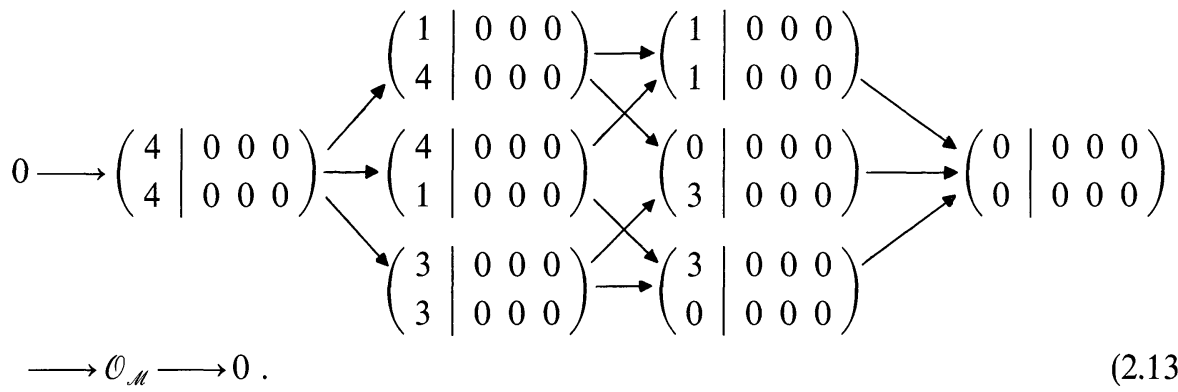

The compound Young-tableau notation which we use specifies the homogeneous bundles over $\mathbb{P}_{x}^{3} \times \mathbb{P}_{y}^{3}$ as follows. The upper (lower) row corresponds to $\mathbb{P}_{x}^{3}\left(\mathbb{P}_{y}^{3}\right)$. Reading each of these from left to right, we list the $U(1)$ charge and then the number of boxes in the lower, middle and upper row of the corresponding $U(3)$ Young-tableau.

Using the "tic-tac-toe" algorithm (Appendix B), we find that the cohomology on $\mathbb{P}^{3} \times \mathbb{P}^{3}$ vanishes for most of the bundles occurring in (2.13). In fact, there are only two non-vanishing contributions and the $0^{\text {th }}$ level of the spectral sequence 
accompanying $(2.13)$ is

\begin{tabular}{|c|c|c|c|c|}
\hline$\wedge^{3} \mathscr{E}^{*}$ & $\wedge^{2} \mathscr{E} *$ & $\mathscr{E}^{*}$ & $\mathcal{O}_{\mathscr{W}}$ & $\mathcal{O}_{\mathscr{M}}$ \\
\hline 0 & 0 & 0 & $\left(\begin{array}{llll}0 & 0 & 0 & 0 \\
0 & 0 & 0 & 0\end{array}\right)$. & $-H^{0}(\mathscr{M})$ \\
\hline 0 & 0 & 0 & 0 & $H^{1}(\mathscr{M})$ \\
\hline 0 & 0 & 0 & 0 & $H^{2}(\mathscr{M})$ \\
\hline 0 & 0 & 0 & $0 \ldots$ & $\triangleright H^{3}(\mathscr{M})$ \\
\hline 0 & 0 & 0. & 0 & $\equiv 0$ \\
\hline 0 & 0. & 0 & 0 & $\equiv 0$ \\
\hline$\left(\begin{array}{llll}1 & 1 & 1 & 1 \\
1 & 1 & 1 & 1\end{array}\right.$ & 0 & 0 & 0 & $\equiv 0$ \\
\hline
\end{tabular}

It is easy to see that there can be no non-trivial differentials $d_{k}$ here. (Acting $k+1$ steps to the right and $k$ steps up, for every $k=0, \ldots, 3$, all $d_{k}$ 's have zero domain or zero image.)

Using the relation (2.12), depicted in the above diagram by the dotted arrows, and that $H^{0, q}(\mathscr{M})=H^{q}\left(\mathscr{M}, \mathcal{O}_{\mathscr{M}}\right)$, we recover

$$
\begin{aligned}
H^{0,1}(\mathscr{M}) & =H^{0,2}(\mathscr{M})=0, \\
\operatorname{dim} H^{0,0}(\mathscr{M}) & =\operatorname{dim} H^{0,3}(\mathscr{M})=1 .
\end{aligned}
$$

Moreover, $H^{0,0}(\mathscr{M})=\left(\begin{array}{llll}0 & 0 & 0 & 0 \\ 0 & 0 & 0 & 0\end{array}\right)$ is represented by complex scalars while

$$
H^{0,3}(\mathscr{M})=\left(\begin{array}{llll}
1 & 1 & 1 & 1 \\
1 & 1 & 1 & 1
\end{array}\right) \approx\left\{\lambda \varepsilon^{a b c d} \varepsilon^{\alpha \beta \gamma \delta}, \lambda \in \mathbb{C}\right\} .
$$

Now we recall the formula from ref. [11]

$$
\Omega=\oint_{\Gamma_{f}} \oint_{\Gamma_{g}} \oint_{\Gamma_{h}} \frac{\varepsilon_{a b c d} x^{a} d x^{b} d x^{c} d x^{d} \varepsilon_{\alpha \beta \gamma \delta} y^{\alpha} d y^{\beta} d y^{\gamma} d y^{\delta}}{f(x) g(y) h(x, y)}
$$

for the holomorphic $(3,0)$-form, where $\Gamma_{f}$ is a contour in $\mathbb{P}^{3} \times \mathbb{P}^{3}$ around the hypersurface $f(x)=0, \Gamma_{g}$ around $g(y)=0$ and $\Gamma_{h}$ around $h(x, y)=0$. As a tensor, $\Omega$ is represented by its tensor coefficient $\varepsilon_{a b c d} \varepsilon_{\alpha \beta \gamma \delta}$. (The three-fold contour integration is necessary to reduce the $(6,0)$-form on $\mathbb{P}_{x}^{3} \times \mathbb{P}_{y}^{3}$ to a $(3,0)$-form on $\mathscr{M}$ while division by $f(x), g(y)$ and $h(x, y)$ creates poles precisely at $\mathscr{M}$, so that the integrals are non-vanishing and are readily evaluated by residues.)

Comparing the relations (2.15) and (2.14), we see that the duality between the holomorphic $(3,0)$-form and the antiholomorphic $(0,3)$-form is reflected in the contravariancy of the two respective tensor coefficients,

$$
\varepsilon_{a b c d} \varepsilon_{\alpha \beta \gamma \delta} \quad \text { v.s. } \varepsilon^{a b c d} \varepsilon^{\alpha \beta \gamma \delta} .
$$

A remark is in order here. One of the purposes of finding explicit tensor representatives of the various cohomology groups is to obtain their transformation properties under the symmetries of $\mathscr{M}$. Typically, the action of these symmetries on $\mathscr{M}$ stems from their action on $\mathbb{P}_{x}^{3} \times \mathbb{P}_{y}^{3}$. Now, a symmetry of $\mathbb{P}_{x}^{3} \times \mathbb{P}_{y}^{3}$ will be 
a symmetry of $\mathscr{M}$ even if the defining polynomials $f(x), g(y)$ and $h(x, y)$ are not left invariant but change only by a multiplicative complex number. It would thus appear that we would always need to find the complete integral representation such as (2.15) for the various forms. Fortunately, this will not be necessary, by virtue of the fact that the coordinates of the embedding space are defined only up to overall scaling. We have that, for example,

$$
\begin{aligned}
\pi_{1}\left(x^{1}, x^{2}, x^{3}, x^{4}\right)=\left(\omega x^{1}, x^{2}, x^{3}, x^{4}\right) & \cong\left(\omega^{2} x^{1}, \omega x^{2}, \omega x^{3}, \omega x^{4}\right) \\
& \cong\left(x^{1}, \omega^{2} x^{2}, \omega^{2} x^{3}, \omega^{2} x^{4}\right),
\end{aligned}
$$

and either of the three equivalent actions can be used. For all the symmetries of the model we consider here, one of these equivalent actions will leave $f(x), g(y)$ and $h(x, y)$ invariant. With this in mind, it should be clear that the various forms are indeed unambiguously represented merely by the tensor coefficients which we shall list. The interested reader should have no problem establishing the complete integral formula in each case.

Finally, we shall also use Serre duality

$$
H^{q}(X, \mathscr{V})^{*}=H^{\operatorname{dim} X-q}\left(X, \mathscr{V}^{*} \otimes \mathscr{K}_{X}\right)
$$

where $\mathscr{K}_{X} \stackrel{\text { def }}{=} \operatorname{det} \mathscr{T}_{X}^{*}$ is the canonical bundle of the manifold $X$. Since in our case

$$
\begin{aligned}
\mathscr{K}_{M}=\left(\begin{array}{c|ccc}
-1 & -1 & -1 & -1 \\
-1 & -1 & -1 & -1
\end{array}\right)[16] \text { and } \\
\qquad\left(\begin{array}{llll}
a & b & c & d \\
\alpha & \beta & \gamma & \delta
\end{array}\right)^{*}=\left(\begin{array}{llll}
-d & -c & -b & -a \\
-\delta & -\gamma & -\beta & -\alpha
\end{array}\right),
\end{aligned}
$$

taking the dual of the Serre duality formula and replacing $\mathscr{V}$ with $\mathscr{V}^{*}$, we obtain

$$
H^{q}\left(\mathscr{M}, \mathscr{V}^{*}\right)=H^{3-q}(\mathscr{M}, \mathscr{V})^{*} \otimes\left(\begin{array}{llll}
1 & 1 & 1 & 1 \\
1 & 1 & 1 & 1
\end{array}\right)
$$

\section{Charged Matter}

We first determine representatives for the $27 \mathrm{~s}$ and the $27 *$ s, i.e., for elements of $H^{1}\left(\mathscr{M}, \mathscr{T}_{\mathscr{M}}\right)$ and $H^{1}\left(\mathscr{M}, \mathscr{T}_{\mathscr{M}}^{*}\right)$. Now, the former of these and $H^{2}\left(\mathscr{M}, \mathscr{T}_{\mathscr{M}}\right)$, which is dual $H^{1}\left(\mathscr{M}, \mathscr{T}_{\mathscr{M}}^{*}\right)$, occur in the sequence (2.6) and are related thereby to the $\mathscr{T}_{W^{-}}$ and $\mathscr{E}$-valued cohomology on $\mathscr{M}$. These, in turn, are determined using the spectral sequences obtained from (2.13) when tensored, one by one, with each irreducible component in

$$
\begin{aligned}
\mathscr{E} & =\left(\begin{array}{r|lll}
-3 & 0 & 0 & 0 \\
0 & 0 & 0 & 0
\end{array}\right) \oplus\left(\begin{array}{r|lll}
0 & 0 & 0 & 0 \\
-3 & 0 & 0 & 0
\end{array}\right) \oplus\left(\begin{array}{r|l|lll}
-1 & 0 & 0 & 0 \\
-1 & 0 & 0 & 0
\end{array}\right), \\
\mathscr{T}_{\mathscr{W}} & =\left(\begin{array}{r|lll}
-1 & 0 & 0 & 1 \\
0 & 0 & 0 & 0
\end{array}\right) \oplus\left(\begin{array}{r|lll}
0 & 0 & 0 & 0 \\
-1 & 0 & 0 & 1
\end{array}\right) .
\end{aligned}
$$

For each of these five bundles, repeated use of the "tic-tac-toe" algorithm in Appendix $\mathrm{B}$ allows one to obtain the $0^{\text {th }}$ level of the accompanying spectral sequence. Following then the spectral sequence algorithm in Appendix C, it is straightforward to determine the cohomology on $\mathscr{M}$, valued in the five bundles in (3.1): 


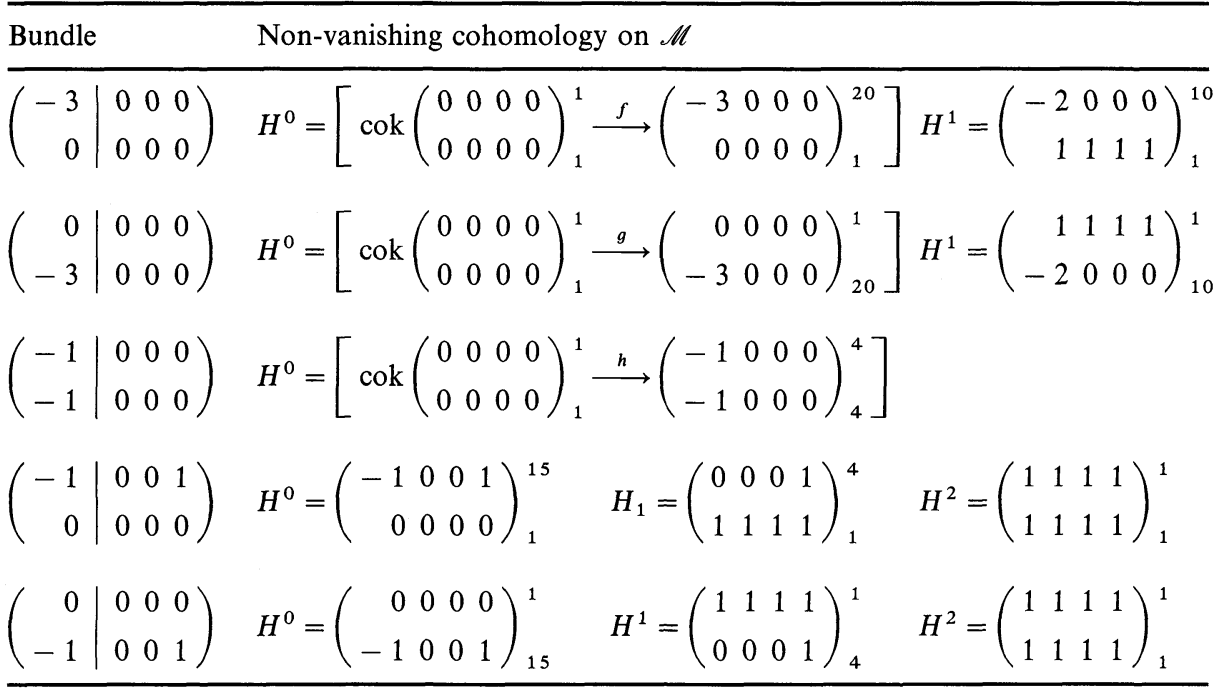

The cokernel of a map $A \stackrel{\xi}{\rightarrow} B$, denoted $\operatorname{cok}(\xi)$, is defined to be the quotient $B / \xi(A)$.

Plugging these into the long exact sequence (2.6), we immediately find

$$
0 \rightarrow H^{3}\left(\mathscr{M}, \mathscr{T}_{\mathscr{M}}\right) \rightarrow 0, \Rightarrow H^{3}\left(\mathscr{M}, \mathscr{T}_{\mathscr{M}}\right) \equiv H^{2,3}(\mathscr{M})=0,
$$

as expected, since $b_{2,3}=0$. The rest of the sequence does not break up this easily:

$$
\begin{aligned}
& 0 \longrightarrow H^{0}\left(\mathscr{M}, \mathscr{T}_{\mathscr{M}}\right) \longrightarrow\left(\begin{array}{rrrr}
-1 & 0 & 0 & 1 \\
0 & 0 & 0 & 0
\end{array}\right) \\
& \left(\begin{array}{rrrr}
0 & 0 & 0 & 0 \\
-1 & 0 & 0 & 0
\end{array}\right) \\
& \stackrel{j_{0}}{\longrightarrow}\left[\operatorname{cok} 3\left(\begin{array}{llll}
0 & 0 & 0 & 0 \\
0 & 0 & 0 & 0
\end{array}\right) \longrightarrow\left(\begin{array}{rrrr}
-3 & 0 & 0 & 1 \\
0 & 0 & 0 & 0
\end{array}\right)\right]
\end{aligned}
$$

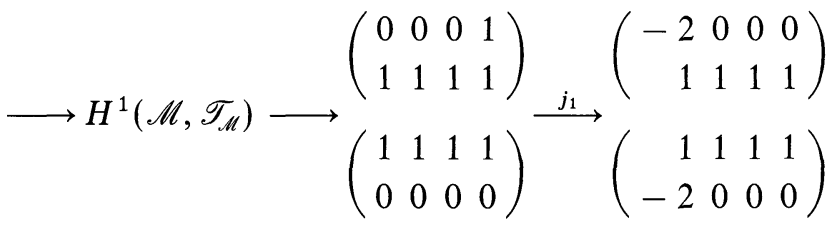

$$
\begin{aligned}
& \longrightarrow H^{2}\left(\mathscr{M}, \mathscr{T}_{\mathscr{M}}\right) \longrightarrow 2\left(\begin{array}{llll}
1 & 1 & 1 & 1 \\
1 & 1 & 1 & 1
\end{array}\right) \longrightarrow 0 \text {. }
\end{aligned}
$$


To save some space, we often stack Young tableaux instead of writing a direct sum.

It is the explicit information encoded in the Young tableaux which enables us to determine the action of the maps $j_{0}$ and $j_{1}$. The action of $j_{1}$ is discerned from considering

$$
\left(\begin{array}{llll}
0 & 0 & 0 & 1 \\
1 & 1 & 1 & 1
\end{array}\right) \stackrel{f}{\longrightarrow}\left(\begin{array}{rrrr}
-2 & 0 & 0 & 0 \\
1 & 1 & 1 & 1
\end{array}\right): f_{a b c}:\left(\varepsilon^{\alpha \beta \gamma \delta} \phi^{a}\right) \mapsto\left(\varepsilon^{\alpha \beta \gamma \delta} \varphi_{(b c)}\right) .
$$

With our choice of $f_{a b c}$, this clearly assigns $\phi^{a} \mapsto \varphi_{(a a)}, a=1,2,3,4$, annihilating none of the $\phi^{a}$. The analogous is true of the "other half" of $j_{1}$ and we conclude that $j_{1}$ has no kernel, whence the long exact sequence (3.2) breaks up so that its second row becomes

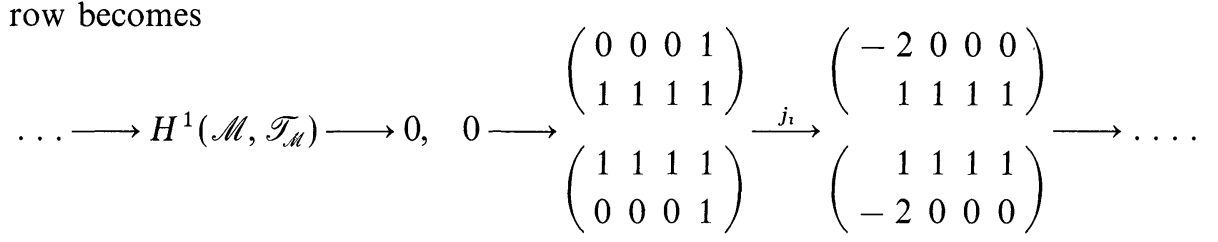

On the other hand, the cokernel of $j_{1}$

$$
\left\{\left(\begin{array}{rrrr}
-2 & 0 & 0 & 0 \\
1 & 1 & 1 & 1
\end{array}\right)_{1}^{10} / f\left(\begin{array}{llll}
0 & 0 & 0 & 1 \\
1 & 1 & 1 & 1
\end{array}\right)_{1}^{4}\right\}_{6} \oplus\left\{\left(\begin{array}{rrrr}
1 & 1 & 1 & 1 \\
-2 & 0 & 0 & 0
\end{array}\right)_{10}^{1} / g\left(\begin{array}{llll}
1 & 1 & 1 & 1 \\
0 & 0 & 0 & 1
\end{array}\right)_{4}^{1}\right\}_{6}
$$

contributes to $H^{2}\left(\mathscr{M}, T_{\mathscr{M}}\right)$ along with $2\left(\begin{array}{llll}1 & 1 & 1 & 1 \\ 1 & 1 & 1 & 1\end{array}\right)$ from the fourth row. Since $H^{2}\left(\mathscr{M}, \mathscr{T}_{\mathscr{M}}\right)$ is dual to $H^{1}\left(\mathscr{M}, \mathscr{T}_{\mathscr{M}}^{*}\right)=H^{1,1}(\mathscr{M})$, we can derive our parametrization of the $(1,1)$-forms on $\mathscr{M}$ by taking duals. For example,

$$
\begin{aligned}
& \left\{\left(\begin{array}{rrrr}
-2 & 0 & 0 & 0 \\
1 & 1 & 1 & 1
\end{array}\right)_{1}^{10} / f\left(\begin{array}{llll}
0 & 0 & 0 & 1 \\
1 & 1 & 1 & 1
\end{array}\right)_{1}^{4}\right\}^{*} \\
& \quad=\left[\operatorname{ker}\left(\begin{array}{llll}
1 & 1 & 1 & 3 \\
0 & 0 & 0 & 0
\end{array}\right)_{1}^{10} \stackrel{f}{\longrightarrow}\left(\begin{array}{llll}
0 & 1 & 1 & 1 \\
0 & 0 & 0 & 0
\end{array}\right)_{1}^{4}\right] .
\end{aligned}
$$

\begin{tabular}{|c|c|}
\hline Contribution to cohomology & Representatives (total of 14) \\
\hline $\operatorname{ker}\left(\begin{array}{llll}1 & 1 & 1 & 3 \\
0 & 0 & 0 & 0\end{array}\right)_{1}^{10} \stackrel{f}{\longrightarrow}\left(\begin{array}{llll}0 & 1 & 1 & 1 \\
0 & 0 & 0 & 0\end{array}\right)_{1}^{4}$ & $\left\{\varepsilon \phi^{(a b)}: f_{a b c} \varepsilon \phi^{(a b)}=0\right\}_{6} \approx\left\{\varepsilon \phi^{(a b)}, a \neq b\right\}_{6}$ \\
\hline $\operatorname{ker}\left(\begin{array}{llll}0 & 0 & 0 & 0 \\
1 & 1 & 1 & 3\end{array}\right)_{10}^{1} \stackrel{g}{\longrightarrow}\left(\begin{array}{llll}0 & 0 & 0 & 0 \\
0 & 1 & 1 & 1\end{array}\right)_{4}^{1}$ & $\left\{\underline{\varepsilon} \phi^{(\alpha \beta)}: g_{\alpha \beta \gamma} \underline{\varepsilon} \phi^{(\alpha \beta)}=0\right\}_{6} \approx\left\{\underline{\varepsilon} \phi^{(\alpha \beta)}, \alpha \neq \beta\right\}_{6}$ \\
\hline$\left(\begin{array}{llll}0 & 0 & 0 & 0 \\
0 & 0 & 0 & 0\end{array}\right)_{1}^{1} \oplus\left(\begin{array}{llll}0 & 0 & 0 & 0 \\
0 & 0 & 0 & 0\end{array}\right)_{1}^{1}$ & (Kähler forms on $\mathbb{P}_{x}^{3} \times \mathbb{P}_{y}^{3}$ ) \\
\hline
\end{tabular}

The results are collected in Table 1.

Table 1. Contributions to $H^{1}\left(\mathscr{M}, \mathscr{T}_{\mathscr{M}}^{*}\right)=H^{1,1}(\mathscr{M})$ and representatives 
Since $\operatorname{ker} j_{1}=0$, the first part of the long exact sequence (3.2) breaks off:

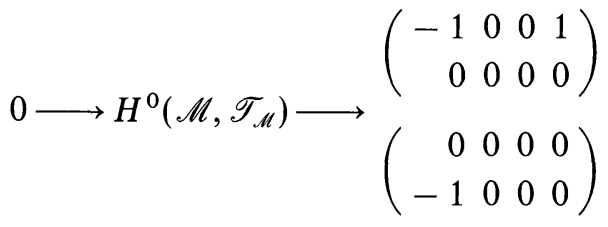

$$
\begin{aligned}
& \stackrel{j_{0}}{\longrightarrow}\left[\operatorname{cok} 3\left(\begin{array}{llll}
0 & 0 & 0 & 0 \\
0 & 0 & 0 & 0
\end{array}\right) \longrightarrow\left(\begin{array}{rrrr}
-3 & 0 & 0 & 1 \\
0 & 0 & 0 & 0
\end{array}\right)\right] \\
& \longrightarrow H^{1}\left(\mathscr{M}, \mathscr{T}_{\mathscr{M}}\right) \longrightarrow 0
\end{aligned}
$$

Consider again "half" of the action of $j_{0}$ :

$$
\left(\begin{array}{rrrr}
-1 & 0 & 0 & 1 \\
0 & 0 & 0 & 0
\end{array}\right)\left\{\begin{array}{l}
\stackrel{f}{\longrightarrow}\left(\begin{array}{rrrr}
-3 & 0 & 0 & 0 \\
0 & 0 & 0 & 0
\end{array}\right): f_{(a b c} \lambda_{d)}{ }^{a} \mapsto \varphi_{(b c d)}, \\
\stackrel{h}{\longrightarrow}\left(\begin{array}{rrrr}
-1 & 0 & 0 & 0 \\
-1 & 0 & 0 & 0
\end{array}\right): h_{a \alpha} \lambda_{d}{ }^{a} \mapsto \varphi_{d \alpha},
\end{array} \quad \operatorname{tr}[\lambda]=\lambda_{a}{ }^{a}=0\right.
$$

No component of $\lambda_{a}{ }^{b}$ is annihilated by both $f$ and $h$ and since the analogous is true of the "other half" of $j_{0}, \operatorname{ker} j_{0}=H^{0}\left(\mathscr{M}, \mathscr{T}_{\mathscr{M}}\right)$ vanishes. $H^{1}\left(\mathscr{M}, \mathscr{T}_{\mathscr{M}}\right)$ is then the combined cokernel of $j_{0}$.

Unlike the situation with $H^{2}\left(\mathscr{M}, \mathscr{T}_{\mathscr{M}}\right)$, this time there is an ambiguity. The cokernel of the combined mapping $j_{0}=f+g+h$ is parametrized by

$$
\begin{aligned}
\varphi_{(a b c)} & \cong \varphi_{(a b c)}+\theta_{f} f_{a b c}+\lambda_{\left({ }^{d}\right.} f_{b c d)}, \\
\varphi_{a \beta} & \cong \varphi_{a \beta}+\theta_{h} h_{a \beta}+\lambda_{a}^{b} h_{b \beta}+\lambda_{\beta}{ }^{\alpha} h_{a \alpha}, \\
\varphi_{(\alpha \beta \gamma)} & \cong \varphi_{(\alpha \beta \gamma)}+\theta_{g} g_{\alpha \beta \gamma}+\lambda_{(\alpha}{ }^{\delta} f_{\beta \gamma \delta)} .
\end{aligned}
$$

We can use the three $\theta$ 's and the two traceless matrices $\lambda_{a}{ }^{b}$ and $\lambda_{\alpha}{ }^{\beta}$ to "gauge away" a total of 33 components among the $20+16+20$ in $\left\{\phi_{(a b c)}, \varphi_{a \beta}, \varphi_{(\alpha \beta \gamma)}\right\}$. All particular choices are equivalent and they lead to a variety of equivalent representatives for this cokernel; Table 2 lists one of them. Note that any two tensor components which are equivalent representatives of the quotient (3.3) necessarily transform identically under any symmetry of $\mathscr{M}$. Indeed, this is a general feature of all equivalence relations and follows from the covariance of the $U(n)$-tensor algebra. However, the opposite is not necessarily true, i.e. tensor components which transform the same do not have to be equivalent. 
Table 2. Contributions to $H^{1}\left(\mathscr{M}, \mathscr{T}_{\mathscr{M}}\right) \approx H^{2,1}(\mathscr{M})$ and representatives

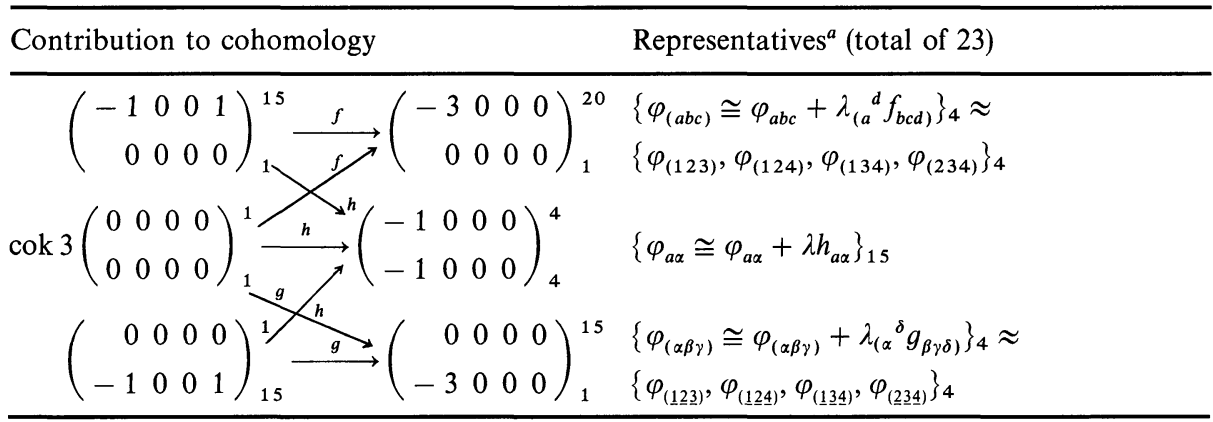

${ }^{a}$ The slanted $h$ maps induce a variety of equivalent representatives; see text

\section{Chargeless Matter}

While conceptually the same as the foregoing, the computation of $H^{1}\left(\mathscr{M}\right.$, End $\left.\mathscr{T}_{\mathscr{M}}\right)$ is much more tedious and involved for several reasons. Firstly, since End $\mathscr{T}_{\mathscr{M}}$ is the bundle of traceless endomorphisms of $\mathscr{T}_{\mathscr{M}}$, it is found in the product

$$
\mathscr{T}_{\mathscr{M}} \otimes \mathscr{T}_{\mathscr{M}}^{*}=\text { End } \mathscr{T}_{\mathcal{M}} \oplus \mathbb{C} .
$$

Related to this, we quote the following general result

Theorem [24]. For a stable holomorphic vector bundle $\mathscr{V}$ over a compact projective manifold $X$,

$$
H^{0}(X, \text { End } \mathscr{V})=0 .
$$

Since the tangent bundle of a Calabi-Yau manifold is stable, $H^{0}\left(\mathscr{M}\right.$, End $\left.\mathscr{T}_{\mathscr{M}}\right)=0$. Because End $\mathscr{T}_{\mathscr{M}}$ is self-dual and the first Chern class of $\mathscr{M}$ vanishes, (i.e., $\mathscr{K}_{\mathscr{M}} \approx \mathbb{C}$ ), by Eq. (2.16) $H^{q}\left(\mathscr{M}\right.$, End $\left.\mathscr{T}_{\mathscr{M}}\right)$ is dual to $H^{3-q}\left(\mathscr{M}\right.$, End $\left.\mathscr{T}_{\mathscr{M}}\right)$ so $q=0,1$ suffices. Then, by Eq. (4.1),

$$
H^{q}\left(\mathscr{M}, \mathscr{T}_{\mathscr{M}} \otimes \mathscr{T}_{\mathscr{M}}^{*}\right)=H^{q}\left(\mathscr{M}, \text { End } \mathscr{T}_{\mathscr{M}}\right) \oplus H^{q}(\mathscr{M}, \mathbb{C})=\left\{\begin{array}{l}
H^{0}(\mathscr{M}), \\
H^{1}\left(\mathscr{M}, \text { End } \mathscr{T}_{\mathscr{M}}\right) .
\end{array}\right.
$$

For $q=1$, we have used that $H^{1}(\mathscr{M}, \mathbb{C})=0$ for manifolds of precisely $S U(3)$ holonomy. In view of these relations, we shall compute the $\mathscr{T}_{\mathcal{M}} \otimes \mathscr{T}^{*}$-valued cohomology and determined $H^{*}\left(\mathscr{M}\right.$, End $\left.\mathscr{T}_{\mathrm{M}}\right)$ from there. We emphasize, however, that our computations do not rely on the above theorem, in fact we prove it for our particular case.

4.1. Polynomial Deformations. Before we proceed with the spectral sequences, let us recall the deformation theoretic result for $H^{1}\left(\mathscr{M}\right.$, End $\left.\mathscr{T}_{\mathscr{M}}\right) . \mathscr{T}_{\mathscr{M}}$-valued 1-forms can (often) be represented by non-trivial deformations of the defining polynomials. In view of the relation (4.1), End $\mathscr{T}_{M}$-valued 1-forms should feature an extra $\mathscr{T}_{\mathscr{M}}^{*}$ factor and could therefore correspond to non-trivial deformations of the differentials of the defining polynomials. Deformations of

$$
d f(x)=d x^{a} f_{a b c} x^{b} x^{c}
$$


would be covered by differentials of the form

$$
d x^{a} \phi_{a(b c)} x^{b} x^{c}: \phi_{a(b c)}+\phi_{b(c a)}+\phi_{c(a b)} \equiv 0 \text {. }
$$

(The totally symmetric tensor is used up to represent deformations $f(x)$ for $H^{1}\left(\mathscr{M}, \mathscr{T}_{\mathscr{M}}\right)$.) Indeed,

$$
\phi_{a(b c)} \text { and } \varphi_{\alpha(\beta \gamma)}
$$

are precisely the contributions to $H^{1}\left(\mathscr{M}\right.$, End $\left.\mathscr{T}_{\mathscr{M}}\right)$ expected by the arguments of ref. [10].

The result (4.3) already satisfies the lower bound $\operatorname{dim} H^{1}\left(\mathscr{M}\right.$, End $\left.\mathscr{T}_{\mathscr{M}}\right) \geqq b_{2,1}$ of ref. [14] but from ref. $[16,17]$ we know that there are more End $\mathscr{T}_{\mathscr{M}}$-valued forms and we now proceed to describe the complete result.

4.2. The Outline of the Spectral Sequence Computation. By tensoring Seq. (2.3) with $\mathscr{T}_{\mathscr{M}}^{*}$ and Seq. (2.5) with $\mathscr{E}$ and with $\mathscr{T}_{\mathscr{W}}$ respectively, we obtain three short exact sequences which fit together into the diagram

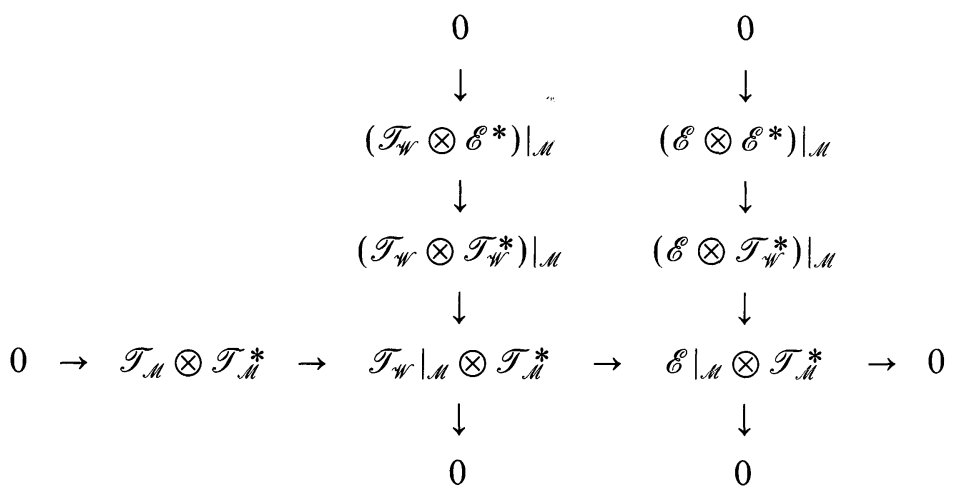

with exact rows and columns. Thus the vector bundle $\mathscr{T}_{\mathscr{M}} \otimes \mathscr{T}_{\mathscr{M}}^{*}$ over $\mathscr{M}$, intrinsic to $\mathscr{M}$, is related to the restriction to $\mathscr{M}$ of various vector bundles over $\mathscr{W}$.

The computation proceeds as follows: 1) using spectral sequences, determine the $\mathscr{T}_{\mathscr{W}} \otimes \mathscr{E}^{*}$-, $\mathscr{E} \otimes \mathscr{E}^{*}$-, $\mathscr{T}_{\mathscr{W}} \otimes \mathscr{T}_{\mathscr{W}}^{*}$ - and $\mathscr{E} \otimes \mathscr{T}_{\mathscr{W}}^{*}$-valued cohomology on $\mathscr{M}$.2) Use the two vertical short exact sequences ${ }^{4}$ in the diagram (4.4) to determine the $\mathscr{T}_{\mathscr{W}} \otimes \mathscr{T}_{\mathscr{M}}^{*}$ - and $\mathscr{E}_{\mathscr{M}} \otimes \mathscr{T}_{\mathscr{M}}^{*}$-valued cohomology. 3) Use the short exact sequence in the bottom row of diagram (4.4) to determine the $\mathscr{T}_{\mathscr{M}} \otimes \mathscr{T}_{\mathscr{M}}^{*}$-valued cohomology and thus $H^{*}\left(\mathscr{M}\right.$, End $\left.\mathscr{T}_{\mathscr{M}}\right)$, through the relations (4.2).

In the rest of this section, we shall try to describe this computation in accessible detail (see also Appendices D and E), hoping to provide a useful templet for the enterprising model builder, who will most unlikely be satisfied with the phenomenological specifics of the present choice (2.2). The less patient reader is invited to consult Tables 5, 6, 7 and leap to Sects. 6, 7 and 8 .

\footnotetext{
${ }^{4}$ By common (ab)use of the term "using an exact sequence," we shall also imply that the accompanying long exact cohomology sequence, i.e., the accompanying spectral sequence, is used - without specifying explicitly
} 
4.3. The Bundles over $\mathbb{P}^{3} \times \mathbb{P}^{3}$. Firstly, we need the cohomology on $\mathscr{M}$ valued in the following 18 irreducible bundles over $\mathbb{P}^{3} \times \mathbb{P}^{3}$ :

$$
\begin{aligned}
& \mathscr{E} \otimes \mathscr{E}^{*}=3\left(\begin{array}{l|lll}
0 & 0 & 0 & 0 \\
0 & 0 & 0 & 0
\end{array}\right) \oplus\left(\begin{array}{r|rrr}
-3 & 0 & 0 & 0 \\
3 & 0 & 0 & 0
\end{array}\right) \oplus\left(\begin{array}{r|rrr}
2 & 0 & 0 & 0 \\
-1 & 0 & 0 & 0
\end{array}\right) \oplus\left(\begin{array}{r|rrr}
1 & 0 & 0 & 0 \\
-2 & 0 & 0 & 0
\end{array}\right) \\
& \oplus\left(\begin{array}{r|rrr}
3 & 0 & 0 & 0 \\
-3 & 0 & 0 & 0
\end{array}\right) \oplus\left(\begin{array}{r|rrr}
-1 & 0 & 0 & 0 \\
2 & 0 & 0 & 0
\end{array}\right) \oplus\left(\begin{array}{r|rrr}
-2 & 0 & 0 & 0 \\
1 & 0 & 0 & 0
\end{array}\right), \\
& \mathscr{T}_{\mathscr{W}} \otimes \mathscr{E}^{*}=\left(\begin{array}{l|lll}
2 & 0 & 0 & 1 \\
0 & 0 & 0 & 0
\end{array}\right) \oplus\left(\begin{array}{r|lll}
-1 & 0 & 0 & 1 \\
3 & 0 & 0 & 0
\end{array}\right) \oplus\left(\begin{array}{l|lll}
0 & 0 & 0 & 1 \\
1 & 0 & 0 & 0
\end{array}\right) \\
& \oplus\left(\begin{array}{l|lll}
0 & 0 & 0 & 0 \\
2 & 0 & 0 & 1
\end{array}\right) \oplus\left(\begin{array}{r|lll}
3 & 0 & 0 & 0 \\
-1 & 0 & 0 & 1
\end{array}\right) \oplus\left(\begin{array}{l|lll}
1 & 0 & 0 & 0 \\
0 & 0 & 0 & 1
\end{array}\right), \\
& \mathscr{T}_{\mathscr{W}} \otimes \mathscr{T}_{\mathscr{W}}^{*}=2\left(\begin{array}{l|lll}
0 & 0 & 0 & 0 \\
0 & 0 & 0 & 0
\end{array}\right) \oplus\left(\begin{array}{l|lll}
0 & -1 & 0 & 1 \\
0 & 0 & 0 & 0
\end{array}\right) \oplus\left(\begin{array}{r|rrr}
-1 & 0 & 0 & 1 \\
1 & -1 & 0 & 0
\end{array}\right) \\
& \oplus\left(\begin{array}{l|rrr}
0 & 0 & 0 & 0 \\
0 & -1 & 0 & 1
\end{array}\right) \oplus\left(\begin{array}{r|rrr}
1 & -1 & 0 & 0 \\
-1 & 0 & 0 & 1
\end{array}\right) \text {. }
\end{aligned}
$$

The $\mathscr{E} \otimes \mathscr{T}_{\mathscr{W}}^{*}$-valued cohomology then can be obtained both directly and through Serre duality from the $\mathscr{T}_{\mathscr{W}} \otimes \mathscr{E}^{*}$-valued one. Thus, sequence $(2.13)$ needs to be tensored one-by-one with each of these 18 bundles and the derivation of the $0^{\text {th }}$ level of the accompanying spectral sequence in each case should pose no problem. Determining the actions of all $d_{i}$ is straightforward but rather tedious for some of these 18 cases. Appendix D presents a sample computation for the $\left(\begin{array}{r|rrr}-3 & 0 & 0 & 0 \\ 3 & 0 & 0 & 0\end{array}\right)$ bundle.

To make a cross-check possible, we list the results of this stage in Table 3.

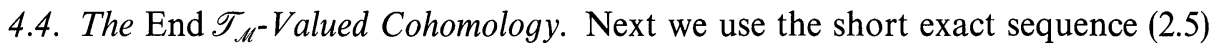
tensored, one by one, with $\mathscr{E}_{f}, \mathscr{E}_{g}, \mathscr{E}_{h}$ and then $\mathscr{T}_{x}$ and $\mathscr{T}_{y}$; these constitute the two vertical exact sequences in the diagram (4.4). From the accompanying long exact cohomology sequences, we obtain a list of cohomology groups (for a sample computation, see Appendix E),

$$
\begin{aligned}
H^{q}\left(\mathscr{M}, \mathscr{T}_{\mathscr{W}} \otimes \mathscr{T}_{\mathscr{M}}^{*}\right) & =H^{q}\left(\mathscr{M}, \mathscr{T}_{x} \otimes \mathscr{T}_{\mathscr{M}}^{*}\right) \oplus H^{q}\left(\mathscr{M}, \mathscr{T}_{y} \otimes \mathscr{T}_{\mathscr{M}}^{*}\right) \\
H^{q}\left(\mathscr{M}, \mathscr{E} \otimes \mathscr{T}_{\mathscr{M}}^{*}\right) & =H^{q}\left(\mathscr{M}, \mathscr{E}_{f} \otimes \mathscr{T}_{\mathscr{M}}^{*}\right) \oplus H^{q}\left(\mathscr{M}_{\mathscr{E}_{g}} \otimes \mathscr{T}_{\mathscr{M}}^{*}\right) \oplus H^{q}\left(\mathscr{M}, \mathscr{E}_{h} \otimes \mathscr{T}_{\mathscr{M}}^{*}\right),
\end{aligned}
$$

which are needed in the final step.

Lastly, we use the short exact sequence in the bottom row of the diagram (4.4). The complete list of all contributions to the cohomology groups occurring in the computation is rather long and little would be gained from a display without going into the tedious details of the analysis. In fact, after some straightforward but lengthy computations similar to those described so far, most of these contributions cancel out anyway and only a few actually go into the $\mathscr{T}_{\mathscr{M}} \otimes \mathscr{T}_{\mathscr{M}}^{*}$-valued cohomology. 
Table 3. The cohomology on $\mathscr{M}$, valued in the bundles that occur in (4.5). The ones which are not listed can be obtained by Serre duality (2.16) and/or the $x \leftrightarrow y$ flip

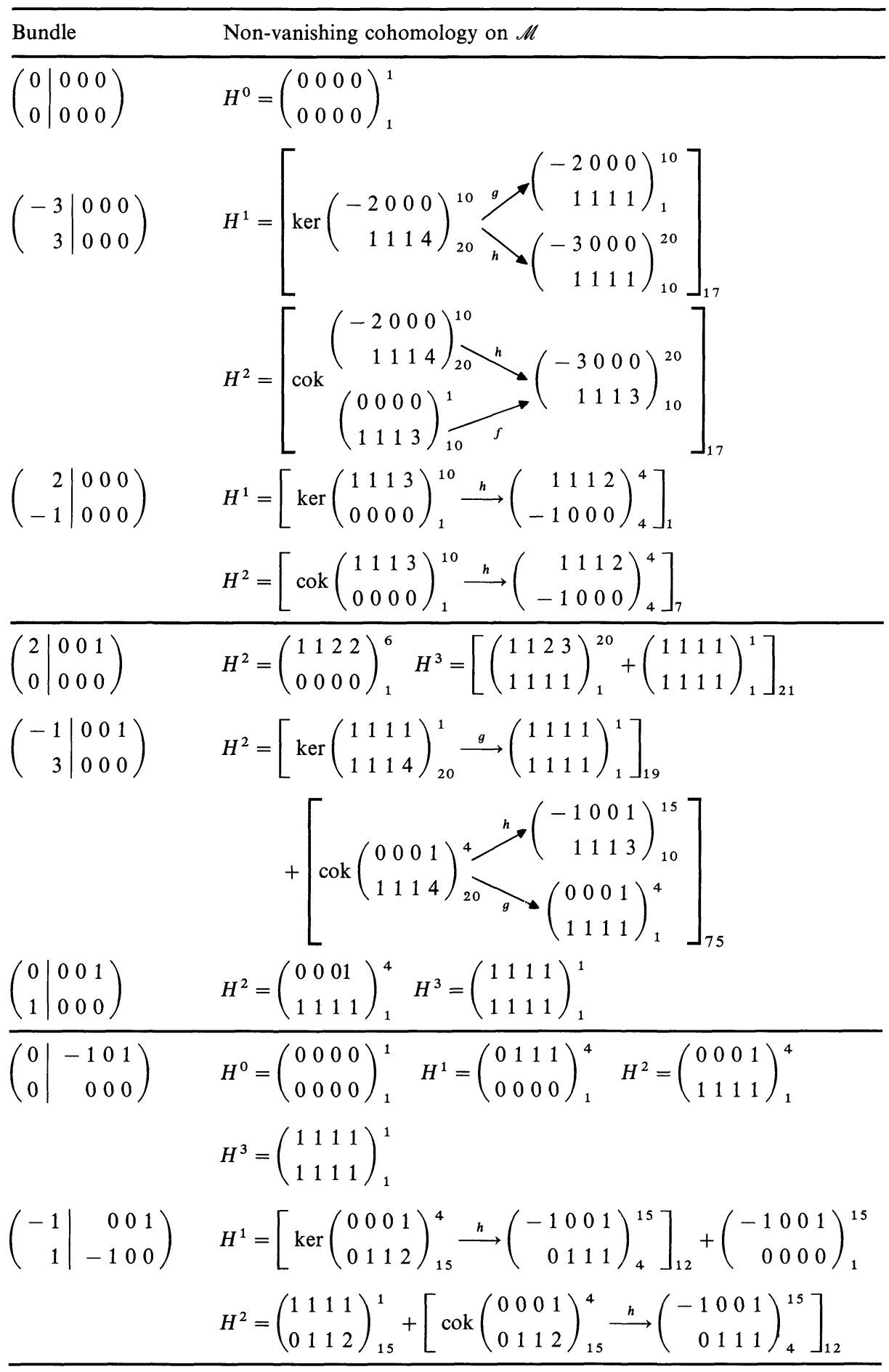


We find $H^{1}\left(\mathscr{M}, \mathscr{T}_{\mathscr{M}} \otimes \mathscr{T}_{\mathscr{M}}^{*}\right)=H^{1}\left(\mathscr{M}\right.$, End $\left.\mathscr{T}_{\mathscr{M}}\right)$ to be 98 -dimensional and spanned by the following tensors (the $\mathbb{P}_{x}^{3} \leftrightarrow \mathbb{P}_{y}^{3}$ flipped contributions are to be added to the subsequent list):

$$
\left(\begin{array}{rrrr}
-2 & -1 & 0 & 0 \\
0 & 0 & 0 & 0
\end{array}\right)_{1}^{20}:\left\{\phi_{a(b c)}\right\}_{20}
$$

which are the only contributions also found by deformation theory (4.3).

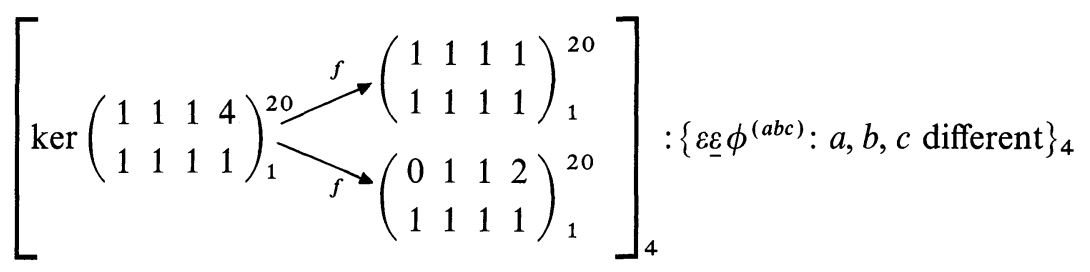

is also easily represented. For the remaining 25 representatives (and their $25 \mathbb{P}_{x}^{3} \leftrightarrow \mathbb{P}_{y}^{3}$ mirror pairs), the tensor constructions are quite more involved and it would take too much space to present them in detail; a set of notes with comments is however available upon request. Here we list the tensor representatives only:

$$
\left\{\varepsilon \phi^{[\hat{a} 4]}\right\}_{3} \quad\left\{\varepsilon \eta^{(\hat{a} \hat{b}) \cdot \hat{\gamma}}, \hat{a} \neq \hat{b}\right\}_{3},
$$

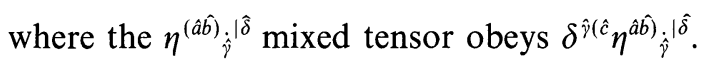

Notation. By a caret, we denote that an index has a restricted range, so that $\hat{a}=1,2,3$ but $\hat{a} \neq 4$. The dot above the $\hat{\gamma}$ in the tensor $\eta$ in (4.8) denotes that this index has to be treated as if lowered by $h_{\hat{a} \hat{\alpha}}=\delta_{\hat{a} \hat{\alpha}}$ to that

$$
\eta^{(\hat{a} \hat{b})} \hat{\hat{\gamma}} \mid \hat{\gamma} \sim \eta^{(\hat{a} \hat{b}) \hat{c} \mid \delta} h_{\hat{c} \hat{\gamma}} .
$$

Now $\eta^{(\hat{a} \hat{b} \hat{c} \hat{\delta} \hat{\delta}}$ vanishes upon total symmetrization of $\hat{a}, \hat{b}$ and $\hat{c}$. The vertical "pipe,"|, simply tells that the rightmost superscript, $\hat{\delta}=1,2,3$, doest not participate in any (anti)symmetrization or trace-zero condition and is merely a free label. In (4.8) then, the trace of $\eta^{(\hat{a} \hat{b})}, \hat{\hat{\gamma}} \hat{\hat{\delta}}$ occurs.

If the third defining equation $h(x, y)=0$ in (2.2) is deformed into

$$
h^{\prime}(x, y)=h(x, y)+x^{4} y^{4}=\sum_{a=\alpha=1}^{4} x^{a} y^{\alpha}=0,
$$

i.e., $h_{44} \neq 0$, the 6 components in (4.8) become replaced by $\left\{\varepsilon \phi^{(a b)}, a \neq b\right\}_{6}$. Lastly,

$$
\begin{aligned}
& \left\{\varepsilon \phi_{4}\right\}_{1} \text {, } \\
& \left\{\varepsilon\left(\sum_{\hat{\gamma}} \eta^{(\hat{a} \hat{b}) \cdot \hat{\gamma}}\right) ; \hat{a}=\hat{b}\right\}_{3}, \\
& \left\{\varepsilon\left(\delta^{\hat{c} \hat{\gamma}} \eta^{(4 \hat{b})} \mid \hat{\gamma} \hat{\delta}-\delta^{\hat{b} \hat{\gamma}} \eta^{(4 \hat{c})} \hat{\gamma}_{\hat{\gamma}}\right)\right\}_{9}, \\
& \left\{\varepsilon\left(\delta_{\hat{c} \hat{\gamma}} \eta_{\hat{a}}^{4 \hat{y}}-\delta_{\hat{a} \hat{\gamma}} \eta_{\hat{c}}^{4 \hat{\gamma}}\right) ; \quad \hat{a} \neq \hat{c}\right\}_{3}, \\
& \left\{\varepsilon\left(\eta^{(\hat{a} \hat{b})}{ }_{4}^{\mid \hat{\beta}}-\eta^{\left(\hat{a} \hat{b}^{\prime}\right)}{ }_{4}^{\mid \hat{\beta}^{\prime}}\right) ; \hat{\beta}=\hat{b}, \hat{\beta}^{\prime}=\hat{b}^{\prime} \text { and } \hat{a}, \hat{b}, \hat{b}^{\prime}, \hat{a} \text { different }\right\}_{3} \text {, }
\end{aligned}
$$

and special to the $R$-symmetric model and cancel out in the computation when the $R$-symmetry is broken, i.e., when $\operatorname{rank}\left[h_{a \alpha}\right]=4$. 


\section{Towards the Three Generations of the Standard Model}

From the point of view of particle physics, the model we have descirbed so far is not realistic. For one thing, it would predict 23 generations of light matter particles and 14 mirror generations. It is by now standard practice to pass to a suitable $\mathbb{Z}_{3}$-quotient model and we now turn to describe the effect of process on the complete matter sector.

5.1. The Two Three-Generation Models. Starting from the smooth Calabi-Yau space $\mathscr{M}$, defined in (2.2), we can define two distinct quotient manifolds by dividing out two inequivalent $\mathbb{Z}_{3}$ actions given in Table 4 . In the second part of Appendix A, we prove that both $\mathscr{M}_{1} \stackrel{\text { def }}{=} \mathscr{M} / \varpi_{1}$ and $\mathscr{M}_{2} \stackrel{\text { def }}{=} \mathscr{M} / \varpi_{2}$ are smooth.

Both $\mathscr{M}_{1}$ and $\mathscr{M}_{2}$ have $\chi_{E}=-6$ and the same number of leptons, quarks and their mirror particles. However, the particular tensors which become identified as quarks and leptons on $\mathscr{M}_{1}$ are different from those on $\mathscr{M}_{2}$. Also, the $34 E_{6} 1$ 's which descend to $\mathscr{M}_{1}$ will be different from those on $\mathscr{M}_{2}$. Also, the structure of (pseudo) symmetries of the interactions in the two associated models is rather different.

The covering space $\mathscr{M}$ possesses the order-6 non-abelian permutation symmetry $S_{3}$ which is generated by:

$$
\begin{aligned}
& \boldsymbol{\sigma}_{12}:\left(x^{1}, x^{2}, x^{3} ; y^{1}, y^{2}, y^{3}\right) \mapsto\left(x^{2}, x^{1}, x^{3} ; y^{2}, y^{1}, y^{3}\right), \\
& \boldsymbol{\sigma}_{23}:\left(x^{1}, x^{2}, x^{3} ; y^{1}, y^{2}, y^{3}\right) \mapsto\left(x^{1}, x^{3}, x^{2} ; y^{1}, y^{3}, y^{2}\right) .
\end{aligned}
$$

Passing to the quotient $\mathscr{M}_{1}$ breaks $S_{3}$ down to the $\mathbb{Z}_{2}$ subgroup generated by $\sigma_{12}$, while $\mathscr{M}_{2}$ inherits the $\mathbb{Z}_{2} \subset S_{3}$ subgroup generated by $\sigma_{23}$.

There is a subtle difference, though, in that $\sigma_{23}$ actually commutes with $\varpi_{2}$, while $\sigma_{12}$ commutes with $\varpi_{1}$ only up to an appropriate twist by $\pi_{1}$ and $\pi_{2}$ (see below). This distinction shows up in the symmetries of the interactions and is eventually of physical importance. In particular, $\boldsymbol{\sigma}_{23}$ gives rise to a $\mathbb{Z}_{2}$ "matter parity" 5 in the three-generation model based on $\mathscr{M}_{2}$. In comparison, in a model based on $\mathscr{M}_{1}$, we could only find a $\mathbb{Z}_{3}$ matter parity. For a recent analysis, see ref. [26]. This difference does not happen to manifest itself among the (renormalizable) Yukawa couplings but is bound to show up at the non-renormalizable level; for more details, see ref. [5].

In Tables 5, 6 and 7, we list the tensor representatives of the $E_{6} 27$ 's, 27*'s and 1's, respectively. Our notation for the quarks differs from the more usual one [9],

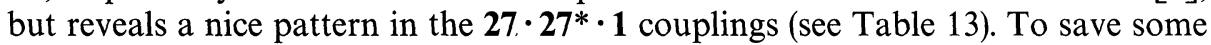
space, we have listed only one half of the 1's; the remaining ones can be recovered using the $\mathbb{P}_{x}^{3} \leftrightarrow \mathbb{P}_{y}^{3}$ symmetry. In Table 7 , the representatives are numbered (first column), for easier reference in the $\sigma_{12}$ and $\sigma_{23}$ columns.

Table 4. The two inequivalent $\mathbb{Z}_{3}$ actions on $\mathscr{M}$

\begin{tabular}{lllllllll}
\hline $\mathbb{Z}_{3}$ & & \multicolumn{8}{c}{ Action on } \\
& $x^{1}$ & $x^{2}$ & $x^{3}$ & $x^{4}$ & $y^{1}$ & $y^{2}$ & $y^{3}$ & $y^{4}$ \\
\hline$\varpi_{1}:$ & 1 & $\omega^{2}$ & $\omega$ & $\omega$ & 1 & $\omega$ & $\omega^{2}$ & $\omega^{2}$ \\
$\varpi_{2}:$ & 1 & $\omega^{2}$ & $\omega^{2}$ & $\omega$ & 1 & $\omega$ & $\omega$ & $\omega^{2}$ \\
\hline
\end{tabular}

$\overline{{ }^{5} \text { For this, } \sigma_{23}}$ needs to be twisted by a symmetry acting on gauge-indices; see ref. [25] 
Table 5. Transformation properties of the matter fields stemming from the 27s. To ensure the $\phi_{1 \underline{1}}+\phi_{2 \underline{2}}+\phi_{3 \underline{3}} \cong 0$ equivalence, we have identified $2 \phi_{2 \underline{2}}=\left(\lambda_{7}-\lambda_{6}\right)$ and $2 \phi_{3 \underline{3}}=-\left(\lambda_{7}+\lambda_{6}\right)$. Slightly different choices are found in the literature

\begin{tabular}{|c|c|c|c|c|c|c|c|c|c|c|}
\hline \multirow[b]{2}{*}{ Tensor } & \multicolumn{2}{|c|}{ Fields } & \multirow[b]{2}{*}{ Norm } & \multicolumn{7}{|c|}{ Symmetries } \\
\hline & $\varpi_{1}$ & $\varpi_{2}$ & & $\pi_{1}$ & $\pi_{2}$ & $\pi_{3}$ & $\mathbf{r}_{x}$ & $\mathbf{r}_{y}$ & $\sigma_{12}$ & $\sigma_{23}$ \\
\hline$\phi_{(123)}$ & $\lambda_{1}$ & $q_{2}$ & $N_{1}$ & $\omega^{2}$ & $\omega^{2}$ & $\omega^{2}$ & 1 & 1 & 1 & 1 \\
\hline$\phi_{(124)}$ & $\lambda_{2}$ & $\lambda_{2}$ & $N_{2}$ & $\omega^{2}$ & $\omega^{2}$ & 1 & $\omega^{2}$ & 1 & 1 & $\phi_{(134)}$ \\
\hline$\phi_{(134)}$ & $Q_{1}$ & $\lambda_{1}$ & $N_{2}$ & $\omega^{2}$ & 1 & $\omega^{2}$ & $\omega^{2}$ & 1 & $\phi_{(234)}$ & $\phi_{(124)}$ \\
\hline$\phi_{(234)}$ & $q_{2}$ & $Q_{1}$ & $N_{2}$ & 1 & $\omega^{2}$ & $\omega^{2}$ & $\omega^{2}$ & 1 & $\phi_{(134)}$ & 1 \\
\hline$\phi_{(\underline{1} 2 \underline{3})}$ & $\lambda_{3}$ & $Q_{2}$ & $N_{1}$ & $\omega$ & $\omega$ & $\omega$ & 1 & 1 & 1 & 1 \\
\hline$\phi_{(124)}$ & $\lambda_{4}$ & $\lambda_{4}$ & $\mathrm{~N}_{2}$ & $\omega$ & $\omega$ & 1 & 1 & $\omega^{2}$ & 1 & $\phi_{(1 \underline{3} \underline{4})}$ \\
\hline$\phi_{(1 \underline{1} \underline{4})}$ & $q_{1}$ & $\lambda_{3}$ & $N_{2}$ & $\omega$ & 1 & $\omega$ & 1 & $\omega^{2}$ & $\phi_{(2 \underline{3} 4)}$ & $\phi_{(124)}$ \\
\hline$\phi_{(2 \underline{3}-4)}$ & $Q_{2}$ & $q_{1}$ & $\mathrm{~N}_{2}$ & 1 & $\omega$ & $\omega$ & 1 & $\omega^{2}$ & $\phi_{(1 \underline{3} 4)}$ & 1 \\
\hline$\phi_{4 \underline{4}}$ & $\lambda_{5}$ & $\lambda_{5}$ & $N_{3}$ & 1 & 1 & 1 & $\omega^{2}$ & $\omega^{2}$ & 1 & 1 \\
\hline & $\lambda_{6}$ & $\lambda_{6}$ & $3 N_{4}$ & 1 & 1 & 1 & 1 & 1 & $\phi_{22}$ & 1 \\
\hline$\left(\phi_{2 \underline{2}}-\phi_{3 \underline{3}}\right)$ & $\lambda_{7}$ & $\lambda_{7}$ & $N_{4}$ & 1 & 1 & 1 & 1 & 1 & $\left(\phi_{1 \underline{1}}-\phi_{3 \underline{3}}\right)$ & -1 \\
\hline$\phi_{12}$ & $q_{3}$ & $q_{3}$ & $N_{5}$ & $\omega^{2}$ & $\omega$ & 1 & 1 & 1 & $\phi_{21}$ & $\phi_{13}$ \\
\hline$\phi_{1 \underline{3}}$ & $Q_{4}$ & $q_{4}$ & $N_{5}$ & $\omega^{2}$ & 1 & $\omega$ & 1 & 1 & $\phi_{2 \underline{3}}$ & $\phi_{1}$ \\
\hline$\phi_{2 \underline{3}}$ & $q_{6}$ & $\lambda_{8}$ & $N_{5}$ & 1 & $\omega^{2}$ & $\omega$ & 1 & 1 & $\phi_{1 \underline{3}}$ & $\phi_{32}^{\underline{1}}$ \\
\hline$\phi_{14}$ & $Q_{5}$ & $Q_{5}$ & $N_{6}$ & $\omega^{2}$ & 1 & 1 & 1 & $\omega^{2}$ & $\phi_{24}$ & 1 \\
\hline$\phi_{24}$ & $q_{7}$ & $q_{7}$ & $N_{6}$ & 1 & $\omega^{2}$ & 1 & 1 & $\omega^{2}$ & $\phi_{14}$ & $\phi_{34}$ \\
\hline$\phi_{34}$ & $\lambda_{8}$ & $q_{6}$ & $N_{6}$ & 1 & 1 & $\omega^{2}$ & 1 & $\omega^{2}$ & 1 & $\phi_{24}$ \\
\hline$\phi_{21}$ & $Q_{3}$ & $Q_{3}$ & $N_{5}$ & $\omega$ & $\omega^{2}$ & 1 & 1 & 1 & $\phi_{12}$ & $\phi_{31}$ \\
\hline$\phi_{31}$ & $q_{4}$ & $Q_{4}$ & $N_{5}$ & $\omega$ & 1 & $\omega^{2}$ & 1 & 1 & $\phi_{32}$ & $\phi_{21}$ \\
\hline$\phi_{32}$ & $Q_{6}$ & $\lambda_{9}$ & $N_{5}$ & 1 & $\omega$ & $\omega^{2}$ & 1 & 1 & $\phi_{3 \underline{1}}$ & $\phi_{2 \underline{3}}$ \\
\hline$\phi_{41}$ & $q_{5}$ & $q_{5}$ & $N_{6}$ & $\omega$ & 1 & 1 & $\omega^{2}$ & 1 & $\phi_{4 \underline{2}}$ & 1 \\
\hline$\phi_{4 \underline{2}}$ & $Q_{7}$ & $Q_{7}$ & $N_{6}$ & 1 & $\omega$ & 1 & $\omega^{2}$ & 1 & $\phi_{41}$ & $\phi_{4 \underline{3}}$ \\
\hline$\phi_{4 \underline{3}}$ & $\lambda_{9}$ & $Q_{6}$ & $N_{6}$ & 1 & 1 & $\omega$ & $\omega^{2}$ & 1 & 1 & $\phi_{42}$ \\
\hline
\end{tabular}

Table 6. Transformation properties of the matter fields stemming from the 27 *s

\begin{tabular}{|c|c|c|c|c|c|c|c|c|c|c|}
\hline \multirow[b]{2}{*}{ Tensor } & \multicolumn{2}{|c|}{ Fields } & \multirow[b]{2}{*}{ Norm } & \multicolumn{7}{|c|}{ Symmetries } \\
\hline & $\varpi_{1}$ & $\varpi_{2}$ & & $\pi_{1}$ & $\pi_{2}$ & $\pi_{3}$ & $\mathbf{r}_{x}$ & $\mathbf{r}_{y}$ & $\sigma_{12}$ & $\sigma_{23}$ \\
\hline$\left(J_{x}+J_{y}\right) / \sqrt{3}$ & $\bar{\lambda}_{1}$ & $\bar{\lambda}_{1}$ & $\bar{N}_{1}$ & 1 & 1 & 1 & 1 & 1 & 1 & 1 \\
\hline$\left(J_{x}-J_{y}\right) / \sqrt{2}$ & $\bar{\lambda}_{2}$ & $\bar{\lambda}_{2}$ & $\bar{N}_{2}$ & 1 & 1 & 1 & 1 & 1 & 1 & 1 \\
\hline$\varepsilon \phi^{(12)}$ & $\bar{\lambda}_{3}$ & $\bar{q}_{4}$ & $\bar{N}_{3}$ & $\omega^{2}$ & $\omega^{2}$ & $\omega$ & $\omega$ & 1 & -1 & $-\varepsilon \phi^{(13)}$ \\
\hline$\varepsilon \phi^{(13)}$ & $\bar{Q}_{2}$ & $\bar{q}_{3}$ & $\bar{N}_{3}$ & $\omega^{2}$ & $\omega$ & $\omega^{2}$ & $\omega$ & 1 & $-\varepsilon \phi^{(23)}$ & $-\varepsilon \phi^{(12)}$ \\
\hline$\varepsilon \phi^{(23)}$ & $-\bar{q}_{3}$ & $-\bar{\lambda}_{3}$ & $\bar{N}_{3}$ & $\omega$ & $\omega^{2}$ & $\omega^{2}$ & $\omega$ & 1 & $-\varepsilon \phi^{(13)}$ & -1 \\
\hline$\varepsilon \phi^{(14)}$ & $-\bar{Q}_{1}$ & $-\bar{\lambda}_{5}$ & $\bar{N}_{4}$ & $\omega^{2}$ & $\omega$ & $\omega$ & $\omega^{2}$ & 1 & $-\varepsilon \phi^{(24)}$ & -1 \\
\hline$\varepsilon \phi^{(24)}$ & $\bar{q}_{4}$ & $\bar{Q}_{1}$ & $\bar{N}_{4}$ & $\omega$ & $\omega^{2}$ & $\omega$ & $\omega^{2}$ & 1 & $-\varepsilon \phi^{(14)}$ & $-\varepsilon \phi^{(34)}$ \\
\hline$\varepsilon \phi^{(34)}$ & $\bar{\lambda}_{5}$ & $\bar{Q}_{2}$ & $\bar{N}_{4}$ & $\omega$ & $\omega$ & $\omega^{2}$ & $\omega^{2}$ & 1 & -1 & $-\varepsilon \phi^{(24)}$ \\
\hline$\underline{\varepsilon} \phi^{(1 \underline{2})}$ & $\bar{\lambda}_{6}$ & $Q_{4}$ & $\bar{N}_{3}$ & $\omega$ & $\omega$ & $\omega^{2}$ & 1 & $\omega$ & -1 & $-\varepsilon \phi^{(\underline{13})}$ \\
\hline$\left.\underline{\varepsilon} \phi^{(1} \underline{3}\right)$ & $\bar{q}_{2}$ & $Q_{3}$ & $\bar{N}_{3}$ & $\omega$ & $\omega^{2}$ & $\omega$ & 1 & $\omega$ & $-\underline{\varepsilon} \phi^{(2 \underline{3})}$ & $-\varepsilon \phi^{(\underline{1} \underline{2})}$ \\
\hline$\underline{\underline{\varepsilon}} \phi^{(2 \underline{3})}$ & $-\bar{Q}_{3}$ & $-\bar{\lambda}_{4}$ & $\bar{N}_{3}$ & $\omega^{2}$ & $\omega$ & $\omega$ & 1 & $\omega$ & $\left.-\bar{\varepsilon} \phi^{(13}\right)$ & -1 \\
\hline$\underline{\varepsilon} \phi^{(144)}$ & $-\bar{q}_{1}$ & $-\bar{\lambda}_{6}$ & $\bar{N}_{4}$ & $\omega$ & $\omega^{2}$ & $\omega^{2}$ & 1 & $\omega^{2}$ & $-\underline{\varepsilon} \phi^{(2 \underline{2} 4)}$ & -1 \\
\hline$\underline{\varepsilon} \phi^{(2 \underline{4})}$ & $\bar{Q}_{4}$ & $\bar{q}_{1}$ & $\bar{N}_{4}^{+}$ & $\omega^{2}$ & $\omega$ & $\omega^{2}$ & 1 & $\omega^{2}$ & $-\underline{\varepsilon} \phi^{(1 \underline{1})}$ & $-\underline{\varepsilon} \phi^{(\underline{34})}$ \\
\hline$\underline{\varepsilon} \phi^{(3 \underline{4})}$ & $\bar{\lambda}_{4}$ & $\bar{q}_{2}$ & $\bar{N}_{4}$ & $\omega^{2}$ & $\omega^{2}$ & $\omega$ & 1 & $\omega^{2}$ & -1 & $-\underline{\varepsilon} \phi^{(2 \underline{2})}$ \\
\hline
\end{tabular}


For completeness, in these Tables, we shall list the transformation properties of the various tensor representatives with respect to both $\sigma_{12}$ and $\sigma_{23}$. In addition, we also list the transformation properties with respect to all phase symmetries

$$
\begin{array}{ll}
\boldsymbol{\pi}_{i}: & x^{i}, y^{i} \\
\mathbf{r}_{x}: & \mapsto \omega x^{i}, \omega^{2} y^{i}, \\
\mathbf{r}_{y}: & \mapsto \omega x^{4},
\end{array}
$$

Note that $\mathbf{r}_{x} \mathbf{r}_{y}^{-1}=\pi_{4}$, which is not an $R$-symmetry; thus, there is actually only one independent $R$-symmetry which we may identify with $R \stackrel{\text { def }}{=} \mathbf{r}_{x} \mathbf{r}_{y}$.

Remark. Note that the tensor representatives for the particles transform in such a way that, for example, $\phi_{(123)} x^{1} x^{2} x^{3}$ is invariant. This unfortunately is opposite to the convention adopted in the recent literature, in which the particle transforms the same as the corresponding polynomial (when a polynomial description exists). This choice is of course irrelevant if one discusses only the $27^{3}$ and (separately) the $27^{* 3}$ couplings. In our case, however, all matter fields are represented in the same

Table 7. Transformation properties of the 1 matter fields. Only half of them are listed here - we omitted those that can be obtained from the listed ones through the $\mathbb{P}_{x}^{3} \leftrightarrow \mathbb{P}_{y}^{3}$ symmetry. A "-

\begin{tabular}{|c|c|c|c|c|c|c|c|c|c|c|c|}
\hline \multirow[b]{2}{*}{ \# } & \multirow{2}{*}{$\begin{array}{l}\text { Tensor } \\
\text { Component }\end{array}$} & \multicolumn{2}{|c|}{ Fields } & \multirow[b]{2}{*}{ Norm } & \multicolumn{7}{|c|}{ Symmetries } \\
\hline & & $\varpi_{1}$ & $\varpi_{2}$ & & $\pi_{1}$ & $\pi_{2}$ & $\pi_{3}$ & $\mathbf{r}_{x}$ & $\mathbf{r}_{y}$ & $\sigma_{12}$ & $\sigma_{23}$ \\
\hline $\begin{array}{l}1 . \\
2 . \\
3 . \\
4 . \\
5 . \\
6 .\end{array}$ & $\begin{array}{c}\left(\phi_{1 \mid(22)}-\phi_{2 \mid(12)}\right) \\
\left(\phi_{1 \mid(33)}-\phi_{3 \mid(13)}\right) \\
\left(\phi_{2 \mid(11)}-\phi_{1 \mid(12)}\right) \\
\left(\phi_{3 \mid(11)}-\phi_{1 \mid(13)}\right) \\
\left(\phi_{2 \mid(33)}-\phi_{3 \mid(23)}\right) \\
\left(\phi_{3 \mid(22)}-\phi_{2 \mid(23)}\right)\end{array}$ & $\begin{array}{l}- \\
- \\
- \\
-\end{array}$ & $\begin{array}{l}- \\
- \\
- \\
s_{1} \\
s_{2}\end{array}$ & $\begin{array}{l}\tilde{N}_{1} \\
\tilde{N}_{1} \\
\tilde{N}_{1} \\
\tilde{N}_{1} \\
\tilde{N}_{1} \\
\tilde{N}_{1}\end{array}$ & $\begin{array}{l}\omega^{2} \\
\omega^{2} \\
\omega \\
\omega \\
1 \\
1\end{array}$ & $\begin{array}{c}\omega \\
1 \\
\omega^{2} \\
1 \\
\omega^{2} \\
\omega\end{array}$ & $\begin{array}{c}1 \\
\omega \\
1 \\
\omega^{2} \\
\omega \\
\omega^{2}\end{array}$ & $\begin{array}{l}1 \\
1 \\
1 \\
1 \\
1 \\
1\end{array}$ & $\begin{array}{l}1 \\
1 \\
1 \\
1 \\
1 \\
1\end{array}$ & $\begin{array}{l}3 . \\
5 . \\
1 . \\
6 . \\
2 . \\
4 .\end{array}$ & $\begin{array}{l}2 . \\
1 . \\
4 . \\
3 . \\
6 . \\
5 .\end{array}$ \\
\hline $\begin{array}{l}7 . \\
8 .\end{array}$ & $\begin{array}{l}\left(\phi_{4 \mid(11)}-\phi_{1 \mid(14)}\right) \\
\left(\phi_{4 \mid(22)}-\phi_{2 \mid(24)}\right)\end{array}$ & - & - & $\begin{array}{l}\tilde{N}_{2} \\
\tilde{N}_{2}\end{array}$ & $\begin{array}{l}\omega \\
1\end{array}$ & $\begin{array}{c}1 \\
\omega\end{array}$ & $\begin{array}{l}1 \\
1\end{array}$ & $\begin{array}{l}\omega^{2} \\
\omega^{2}\end{array}$ & $\begin{array}{l}1 \\
1\end{array}$ & $\begin{array}{l}8 . \\
7 .\end{array}$ & $\begin{array}{l}7 . \\
9 .\end{array}$ \\
\hline 9. & $\left(\phi_{4 \mid(33)}-\phi_{3 \mid(34)}\right)$ & $s_{1}$ & - & $\tilde{N}_{2}$ & 1 & 1 & $\omega$ & $\omega^{2}$ & 1 & 9. & 8. \\
\hline $\begin{array}{l}10 . \\
11 . \\
12 .\end{array}$ & $\begin{array}{c}\left(\phi_{(1 \mid(44)}-\phi_{4 \mid(14)}\right) \\
\left(\phi_{2 \mid(44)}-\phi_{4 \mid(24)}\right) \\
\left(\phi_{3 \mid 44)}-\phi_{4 \mid(34)}\right)\end{array}$ & $\overline{-}$ & - & $\begin{array}{l}\tilde{N}_{3} \\
\tilde{N}_{3} \\
\tilde{N}_{3}\end{array}$ & $\begin{array}{c}\omega^{2} \\
1 \\
1\end{array}$ & $\begin{array}{c}1 \\
\omega^{2} \\
1\end{array}$ & $\begin{array}{c}1 \\
1 \\
\omega^{2}\end{array}$ & $\begin{array}{l}\omega \\
\omega \\
\omega\end{array}$ & $\begin{array}{l}1 \\
1 \\
1\end{array}$ & $\begin{array}{l}11 . \\
10 . \\
12 .\end{array}$ & $\begin{array}{l}10 . \\
12 . \\
11 .\end{array}$ \\
\hline $\begin{array}{l}13 . \\
14 .\end{array}$ & $\begin{array}{l}\phi_{1(23)} \\
\left(\phi_{2 \mid(13)}-\phi_{3 \mid(12)}\right)\end{array}$ & $\begin{array}{l}s_{3} \\
s_{4}\end{array}$ & - & $3 \tilde{N}_{4}$ & $\begin{array}{l}\omega^{2} \\
\omega^{2}\end{array}$ & $\begin{array}{l}\omega^{2} \\
\omega^{2}\end{array}$ & $\begin{array}{l}\omega^{2} \\
\omega^{2}\end{array}$ & $\begin{array}{l}1 \\
1\end{array}$ & $\begin{array}{l}1 \\
1\end{array}$ & $\begin{array}{c}(14 .-13 .) / 2 \\
(313 .+14 .) / 2\end{array}$ & $\begin{array}{r}13 . \\
-14\end{array}$ \\
\hline $\begin{array}{l}15 . \\
16 . \\
17 .\end{array}$ & $\begin{array}{l}\phi_{4(12)} \\
\phi_{4(13)} \\
\phi_{4(23)}\end{array}$ & $\begin{array}{l}s_{5} \\
-\end{array}$ & $\begin{array}{l}s_{5} \\
s_{6} \\
\end{array}$ & $\begin{array}{l}\tilde{N}_{5} \\
\tilde{N}_{5} \\
\tilde{N}_{5}\end{array}$ & $\begin{array}{c}\omega^{2} \\
\omega^{2} \\
1\end{array}$ & $\begin{array}{c}\omega^{2} \\
1 \\
\omega^{2}\end{array}$ & $\begin{array}{c}1 \\
\omega^{2} \\
\omega^{2}\end{array}$ & $\begin{array}{l}\omega^{2} \\
\omega^{2} \\
\omega^{2}\end{array}$ & $\begin{array}{l}1 \\
1 \\
1\end{array}$ & $\begin{array}{l}15 . \\
17 . \\
16 .\end{array}$ & $\begin{array}{l}16 . \\
15 . \\
17 .\end{array}$ \\
\hline $\begin{array}{l}18 . \\
19 . \\
20 .\end{array}$ & $\begin{array}{r}\left(\phi_{1 \mid(24)}-\phi_{2 \mid(14)}\right) \\
\left(\phi_{1 \mid(34)}-\phi_{3 \mid(14)}\right) \\
\left(\phi_{2 \mid(34)}-\phi_{3 \mid(24)}\right.\end{array}$ & $\frac{s_{6}}{-}$ & $\begin{array}{l}s_{3} \\
s_{4} \\
-\end{array}$ & $\begin{array}{l}\tilde{N}_{6} \\
\tilde{N}_{6} \\
\tilde{N}_{6}\end{array}$ & $\begin{array}{l}\omega^{2} \\
\omega^{2} \\
1\end{array}$ & $\begin{array}{l}\omega^{2} \\
1 \\
\omega^{2}\end{array}$ & $\begin{array}{c}1 \\
\omega^{2} \\
\omega^{2}\end{array}$ & $\begin{array}{l}\omega^{2} \\
\omega^{2} \\
\omega^{2}\end{array}$ & $\begin{array}{l}1 \\
1 \\
1\end{array}$ & $\begin{array}{r}-18 . \\
20 . \\
19 .\end{array}$ & $\begin{array}{r}19 . \\
18 . \\
-20\end{array}$ \\
\hline $\begin{array}{l}21 . \\
22 . \\
23 .\end{array}$ & $\begin{array}{l}\left(\varepsilon \phi^{[14]}\right) \\
\left(\varepsilon \phi^{[24]}\right) \\
\left(\varepsilon \phi^{[34]}\right)\end{array}$ & - & $\begin{array}{l}s_{9} \\
-\end{array}$ & $\begin{array}{l}\tilde{N}_{7} \\
\tilde{N}_{7} \\
\tilde{N}_{7}\end{array}$ & $\begin{array}{l}\omega^{2} \\
\omega \\
\omega\end{array}$ & $\begin{array}{l}\omega \\
\omega^{2} \\
\omega\end{array}$ & $\begin{array}{l}\omega \\
\omega \\
\omega^{2}\end{array}$ & $\begin{array}{l}\omega^{2} \\
\omega^{2} \\
\omega^{2}\end{array}$ & $\begin{array}{l}1 \\
1 \\
1\end{array}$ & $\begin{array}{l}-22 \\
-21 \\
-23\end{array}$ & $\begin{array}{l}-21 . \\
-23 . \\
-22 .\end{array}$ \\
\hline
\end{tabular}
entry in the third (fourth) column indicates non-trivial transformation and thus annihilation when modding by $\varpi_{1}\left(\varpi_{2}\right)$

We have used $3 \phi_{a(b c)} \stackrel{\text { def }}{=} 2 \phi_{a \mid(b c)}-\phi_{b|| a c)}-\phi_{c|| a b)}$ and $2 \phi_{a \mid b c)} \stackrel{\text { def }}{=} \phi_{a b c}+\phi_{a c b}$. 
Table 7. (part 2/2)

\begin{tabular}{|c|c|c|c|c|c|c|c|c|c|c|c|}
\hline \multirow[b]{2}{*}{$\#$} & \multirow{2}{*}{$\begin{array}{l}\text { Tensor } \\
\text { Component }\end{array}$} & \multicolumn{2}{|c|}{ Fields } & \multirow[b]{2}{*}{ Norm } & \multicolumn{7}{|c|}{ Symmetries } \\
\hline & & $\varpi_{1}$ & $\varpi_{2}$ & & $\pi_{1}$ & $\pi_{2}$ & $\pi_{3}$ & $\mathbf{r}_{x}$ & $\mathbf{r}_{y}$ & $\sigma_{12}$ & $\sigma_{23}$ \\
\hline 24. & $\varepsilon\left(\Sigma_{\hat{\gamma}} \eta^{(12) \cdot{ }_{i}^{\mid \eta}}\right)$ & $s_{8}$ & - & $\tilde{N}_{8}$ & $\omega^{2}$ & $\omega^{2}$ & $\omega$ & $\omega$ & 1 & -24 & -25 \\
\hline 25. & $\varepsilon\left(\Sigma_{\hat{\gamma}} \eta^{(13)} \cdot \hat{j}\right)$ & - & - & $\tilde{N}_{8}$ & $\omega^{2}$ & $\omega$ & $\omega^{2}$ & $\omega$ & 1 & -26 & -24 \\
\hline 26. & $\varepsilon\left(\sum_{\hat{p}} \eta^{(23)} \cdot \hat{\gamma}\right)$ & - & $s_{10}$ & $\tilde{N}_{8}$ & $\omega$ & $\omega^{2}$ & $\omega^{2}$ & $\omega$ & 1 & -25 & -26 \\
\hline 27. & $\left(\varepsilon \underline{\varepsilon} \phi^{(123)}\right)$ & $s_{9}$ & - & $\tilde{N}_{9}$ & $\omega$ & $\omega$ & $\omega$ & $\omega$ & $\omega$ & 27. & 27. \\
\hline 28. & $\left(\varepsilon \underline{\varepsilon} \phi^{(124)}\right)$ & $s_{10}$ & $s_{7}$ & $\tilde{N}_{10}$ & $\omega$ & $\omega$ & 1 & $\omega^{2}$ & $\omega$ & 28. & 29. \\
\hline 29. & $\left(\varepsilon \underline{\varepsilon} \phi^{(134)}\right)$ & - & $s_{8}$ & $\tilde{N}_{10}$ & $\omega$ & 1 & $\omega$ & $\omega^{2}$ & $\omega$ & 30. & 28 . \\
\hline 30. & $\left(\varepsilon \underline{\varepsilon} \phi^{(234)}\right.$ & - & - & $\tilde{N}_{10}$ & 1 & $\omega$ & $\omega$ & $\omega$ & $\omega$ & 29. & 30. \\
\hline 31. & $\left(\varepsilon \phi_{4} \cong \phi^{[123]}\right)$ & $s_{11}$ & - & $\tilde{N}_{11}$ & $\omega$ & $\omega$ & $\omega$ & 1 & 1 & -31 & -31 \\
\hline 32. & $\varepsilon\left(\Sigma_{\gamma} \eta^{(11)} \cdot \mid \gamma\right)$ & - & - & $\tilde{N}_{12}$ & 1 & $\omega$ & $\omega$ & $\omega$ & 1 & -33 & -32 \\
\hline 33. & $\varepsilon\left(\sum_{\eta} \eta^{(22)} \eta_{j}^{17}\right)$ & - & $s_{11}$ & $\tilde{N}_{12}$ & $\omega$ & 1 & $\omega$ & $\omega$ & 1 & -34 & -30 . \\
\hline 34. & $\varepsilon\left(\sum_{i} \eta^{(33)}, i \gamma\right)$ & $s_{12}$ & $s_{12}$ & $\tilde{N}_{12}$ & $\omega$ & $\omega$ & 1 & $\omega$ & 1 & -34 & -33 \\
\hline 35. & $\varepsilon\left(\eta_{2}^{(41) \mid 1}-\eta_{1}^{(42) \mid 1}\right)$ & - & - & $\tilde{N}_{13}$ & $\omega$ & $\omega^{2}$ & $\omega$ & $\omega^{2}$ & 1 & 37. & -36 \\
\hline 36. & $\varepsilon\left(\eta^{(41){ }_{3} \mid 1}-\eta^{(43) \mid 1}\right)$ & $s_{13}$ & - & $\tilde{N}_{13}$ & $\omega$ & $\omega$ & $\omega^{2}$ & $\omega^{2}$ & 1 & -38 & -35 \\
\hline 37. & $\varepsilon\left(\eta^{(41)^{2}}\left|2-\eta^{(42)}\right| \underline{2}\right)$ & - & $s_{13}$ & $\tilde{N}_{13}$ & $\omega^{2}$ & $\omega$ & $\omega$ & $\omega^{2}$ & 1 & 35. & -39 \\
\hline 38. & 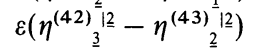 & $s_{14}$ & - & $\tilde{N}_{13}$ & $\omega$ & $\omega$ & $\omega^{2}$ & $\omega^{2}$ & 1 & -36 & 40. \\
\hline 39. & $\varepsilon\left(\eta^{(41)^{2} \mid \underline{3}}-\eta^{(43)^{2} \mid \frac{1}{3}}\right)$ & - & $s_{14}$ & $\tilde{N}_{13}$ & $\omega^{2}$ & $\omega$ & $\omega$ & $\omega^{2}$ & 1 & -40 & -37 \\
\hline 40. & $\varepsilon\left(\eta^{(42)^{3} \mid \frac{3}{3}}-\eta^{(43)^{1}} \frac{13}{2}\right)$ & - & - & $\tilde{N}_{13}$ & $\omega$ & $\omega^{2}$ & $\omega$ & $\omega^{2}$ & 1 & -39 & 38. \\
\hline 41. & $\varepsilon\left(\eta_{3}^{(42) \mid 1}-\eta_{2}^{(43) \mid 1}\right)$ & - & - & $\tilde{N}_{14}$ & 1 & $\omega^{2}$ & $\omega^{2}$ & $\omega^{2}$ & 1 & -42 & 41. \\
\hline 42. & $\varepsilon\left(\eta^{(41)^{\underline{2}} \mid 2}-\eta^{(43)^{2} \mid \frac{1}{2}}\right)$ & - & $s_{15}$ & $\tilde{N}_{14}$ & $\omega^{2}$ & 1 & $\omega^{2}$ & $\omega^{2}$ & 1 & -41 & -43 \\
\hline 43. & 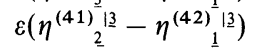 & $s_{15}$ & $s_{16}$ & $\tilde{N}_{14}$ & $\omega^{2}$ & $\omega^{2}$ & 1 & $\omega^{2}$ & 1 & 43. & -42 \\
\hline 44. & $\varepsilon\left(\eta_{4}^{(12) \mid 2}-\eta_{4}^{(13) \mid 3}\right)$ & - & $s_{17}$ & $\tilde{N}_{15}$ & $\omega^{2}$ & $\omega$ & $\omega$ & $\omega$ & $\omega^{2}$ & -45 & 44. \\
\hline 45. & $\varepsilon\left(\eta^{(12)^{4} \mid 1}-\eta^{(23)^{4} \mid 3}\right)$ & - & - & $\tilde{N}_{15}$ & $\omega$ & $\omega^{2}$ & $\omega$ & $\omega$ & $\omega^{2}$ & -44 & -46 \\
\hline 46. & $\varepsilon\left(\eta_{4}^{(13)^{2} \mid 11}-\eta_{4}^{(23)^{2}|2|}\right)$ & $s_{16}$ & - & $\tilde{N}_{15}$ & $\omega$ & $\omega$ & $\omega^{2}$ & $\omega$ & $\omega^{2}$ & 46. & -45 \\
\hline 47. & $\varepsilon\left(\eta_{1}^{42}-\eta_{2}^{41}\right)$ & $s_{17}$ & - & $\tilde{N}_{16}$ & 1 & 1 & $\omega$ & $\omega^{2}$ & 1 & 47. & -48 \\
\hline 48. & $\varepsilon\left(\eta_{1}{ }^{43}-\eta_{3}{ }^{41}\right)$ & - & - & $\tilde{N}_{16}$ & 1 & $\omega$ & 1 & $\omega^{2}$ & 1 & -49 & -47 \\
\hline 49. & $\varepsilon\left(\eta_{2}{ }^{43}-\eta_{3}{ }^{42}\right)$ & - & - & $\tilde{N}_{16}$ & $\omega$ & 1 & 1 & $\omega^{2}$ & 1 & -48 & 49. \\
\hline
\end{tabular}

"language" and so a universal convention was needed. In view of the fact that the 27's may be viewed as the (slowly varying, spacetime dependent) coefficients of the defining polynomials

$$
f(x) \rightarrow\left(f_{a b c}+\phi_{(a b c)}\right) x^{a} x^{b} x^{c},
$$

we find it natural to have the $\phi_{(a b c)}$ transform oppositely to $x^{a} x^{b} x^{c}$. This also agrees with the routine argument that tensor coefficients and coordinates transform in the opposite fashion. This then fixes our convention for the transformation of all other quantities.

5.2. Yukawa Couplings. Without much ado, we record the general formulae for the four types of Yukawa couplings amongst the chiral superfield 27's, 27*'s and 1's.

$$
\left[\int d^{2} \theta \boldsymbol{\Phi}_{i}^{A}(x) \boldsymbol{\Phi}_{j}^{B}(x) \boldsymbol{\Phi}_{k}^{C}(x)\right] d_{A B C}\left[\int_{\mathscr{M}} d^{6} y \Omega^{\overline{\mu \nu} \bar{\rho}} \phi_{\bar{\mu}}^{(i) \alpha}(y) \phi_{\bar{\nu}}^{(j) \beta}(y) \phi_{\bar{\rho}}^{(k) \gamma}(y) \Omega_{\alpha \beta \gamma}\right]
$$


is the $27^{3}$ term, where $d_{A B C}$ is the $E_{6}\left\langle\mathbf{2 7 ^ { 3 }} \mid \mathbf{1}\right\rangle$ Clebsh-Gordan coefficient, and the internal space integral in the second square-bracket is what is commonly called the $27^{3}$-Yukawa coupling. The $\boldsymbol{\Phi}_{i}(x)$ 's represent the 27 spacetime chiral superfields, while $\phi_{\bar{\mu}}^{(i) \alpha}(y)$ 's are the corresponding harmonics over the internal space. Since $\Omega^{\bar{\mu} \bar{v} \bar{\rho}}$ is the same as $\Omega_{\alpha \beta \gamma}$, merely with the indices raised with the Kähler metric, we note that there are two $\Omega$ 's in the above expression.

$$
\left[\int d^{2} \theta \Psi_{a}^{\bar{A}}(x) \Psi_{b}^{\bar{B}}(x) \Psi_{c}^{\bar{C}}(x)\right] d_{\bar{A} \bar{B} \bar{C}}\left[\int_{\mathscr{M}} d^{6} y \Omega^{\bar{\mu} \bar{\nu} \bar{\rho}} \bar{\Omega}^{\alpha \beta \gamma} \psi_{\alpha \bar{\mu}}^{(a)}(y) \psi_{\beta \bar{\nu}}^{(b)}(y) \psi_{\gamma \bar{\rho}}^{(c)}(y)\right]
$$

is the $27^{* 3}$ term, where $d_{\bar{A} \bar{B} \bar{C}}$ is the conjugate of $d_{A B C}$ and the internal space integral in the second square-bracket is what is commonly called the $27^{* 3}$-Yukawa coupling. The $\Psi_{a}(x)$ 's represent the $27^{*}$ spacetime chiral superfield, while $\psi_{\alpha \bar{\mu}}^{(a)}(y)$ 's are the corresponding harmonics over the internal space. Since $\Omega^{\bar{\mu} \bar{\nu}}$ transforms opposite from $\bar{\Omega}^{\alpha \beta \gamma}$, this coupling will transform as if having no $\Omega$ 's; indeed, it can be rewritten witout explicit use of $\Omega$ 's.

The $\mathbf{1}^{3}$ term is

$$
\left[\int d^{2} \theta \Sigma_{p}(x) \Sigma_{q}(x) \Sigma_{r}(x)\right]\left[\int_{\mathscr{M}} d^{6} y \Omega^{\bar{\mu} \bar{\nu} \bar{\rho}} \vartheta_{\bar{\mu} \alpha}^{(p) \beta}(y) \vartheta_{\bar{\nu} \beta}^{(q) \gamma}(y) \vartheta_{\bar{\rho} \gamma}^{(r) \alpha}(y)\right],
$$

where the $d^{6} y$-integral in the second square-bracket is what is commonly called the $\mathbf{1}^{3}$-Yukawa coupling. The $\Sigma_{p}(x)$ 's represent the 1 spacetime chiral superfields, while $\vartheta_{\bar{\mu} \alpha}^{(p) \beta}(y)$ 's are the corresponding harmonics over the internal space. We note that there is only one $\Omega$ in the above expression.

$$
\left[\int d^{2} \theta \Phi_{i}^{A}(x) \Psi_{b}^{\bar{B}}(x) \Sigma_{\boldsymbol{r}}(x)\right] \delta_{A \bar{B}}\left[\int_{\mathscr{M}} d^{6} y \Omega^{\bar{\mu} \bar{\nu} \bar{\rho}} \phi_{\bar{\mu}}^{(i) \alpha}(y) \psi_{\beta \bar{v}}^{(b)}(y) \vartheta_{\bar{\rho} \alpha}^{(\boldsymbol{r}) \beta}(y)\right]
$$

is the mixed, $\mathbf{2 7} \cdot \mathbf{2 7} * \cdot \mathbf{1}$ term, and the $d^{6} y$-integral in the second square-bracket is what is commonly called the $27 \cdot 27 * \cdot 1$-Yukawa coupling. Again, there is only one $\Omega$ in the above expression.

In fact, all four $d^{6} y$ integrals can be rewritten in terms of the respective harmonic $H^{1}\left(\mathscr{M}, \mathscr{T}_{\mathscr{M}}\right)$-, $H^{1}\left(\mathscr{M}, \mathscr{T}_{\mathscr{M}}^{*}\right)$ - and $H^{1}\left(\mathscr{M}\right.$, End $\left.\mathscr{T}_{\mathscr{M}}\right)$-valued $(0,1)$-forms and the holomorphic $(3,0)$-form $\Omega$, whence all products become the skew-symmetric "wedge" products. Since harmonic forms are closed, these integrals are rather "topological" in nature and depend only on the complex structure (represented by the choice of $\Omega$ ), except for (5.7), which are truly topological.

Because of this "topological" nature, these integrals have to be invariant under all symmetries of the internal manifold $\mathscr{M}$ or $\mathscr{M}_{i}$. This includes the so-called $R$-symmetries, which act both on the spacetime part (the first square-brackets) and on the internal space part (the second square-brackets) of the above couplings. Under an $R$-symmetry, both $d^{2} \theta$ and $\Omega$ transform with the same charge, which has to be separately balanced by the spacetime chiral superfields and by the internal space harmonics.

In our case, the $R$-symmetry acts on the internal space as $\mathbf{R} \stackrel{\text { def }}{=} \mathbf{r}_{x} \mathbf{r}_{y}$ (see Eqs. (5.3), (5.4)). In view of Eq. (2.15), $\Omega$ is represented by $\varepsilon_{1234} \varepsilon_{1234}$ and so transfrorms by a phase $\omega^{2}, \omega^{2}$ and $\omega$ under $\mathbf{r}_{x}, \mathbf{r}_{y}$ and $\mathbf{R}$, respectively. The requirement of invariance under $\mathbf{R}$ (and also $\mathbf{r}_{x, y}$ ) of the $d^{6} y$-integrals representing the various couplings will provide a very important selection rule and help us establish our generalization of the Yukawa coupling formula of ref. [11]. 


\section{The $27^{3}$ Yukawa Couplings}

Having obtained tensor representative corresponding to $27 \mathrm{~s}$, we now wish to use this to compute their various couplings and we begin by discussing the $\left(27^{3}\right)$ terms in the superpotential. To see how this can be done, we first note the straightforward relation to polynomial deformations of ref. [11].

6.1. Relation to Polynomial Deformations. In ref. [11], $\mathscr{T}_{\mathcal{M}}$-valued 1 -forms were related to deformations of the defining polynomials modulo the Jacobian ideal (the set of equivalence relations generated by coordinate reparametrizations) of the embedding space $\mathbb{P}^{3} \times \mathbb{P}^{3}$. For the manifold at hand, these are (see also ref. $[9,21]$ )

$$
\begin{aligned}
& q^{i} \simeq q^{i}+X^{a}(x) \partial_{a} p^{i}+Y^{a}(y) \partial_{\alpha} p^{i}+\lambda p^{i}, \\
& p^{i}=f(x), g(y), h(x, y), \quad i=1,2,3 .
\end{aligned}
$$

Here $X^{a}(x)$ are components of a 4-vector of linear combinations of $x$ 's, while $Y^{\alpha}(y)$ are components of a 4-vector of linear combinations of $y^{\prime}$ s. $\partial_{a}$ and $\partial_{\alpha}$ are partial derivatives with respect to $x^{a}$ and $y^{\alpha}$, respectively. The variables $q^{i}$ are polynomials of the degree of $f(x), g(y)$ and $h(x, y)$ for $i=1,2,3$ respectively.

When contracted with homogeneous coordinates $x^{a}$ and $y^{\alpha}$, the tensor representatives from Table 2, with the complete equivalence relations presented in (3.3), are precisely the polynomial deformations $q^{i}$. Thus, our TESS computation explicitly verifies the results of polynomial deformations as also found in ref. [3]. Our aim is now to make use of this correspondence for a translation of the Yukawa coupling algorithm in ref. [11] into the TESS language and then possibly to generalize it.

6.2. Yukawa Couplings as Traces. When $H^{1}\left(\mathscr{M}, \mathscr{T}_{\mathscr{M}}\right)$ is faithfully represented by polynomial deformations, a formula for the $27^{3}$ Yukawa coupling has been derived in ref. [11]. The basic result is that, because of the equivalence relations in (3.3), the product of three polynomial representatives $q^{i}, r^{j}$ and $s^{k}$ turns out to be a multiple of a unique polynomial $Q(x, y)$, modulo the Jacobian ideal:

$$
\begin{aligned}
q^{\left(i_{r}{ }^{j} s^{k)} \simeq\right.} & \delta_{(123)}^{(i j k)} \kappa_{q r s} Q(x, y) \\
& +X^{a(i j}(x, y) \partial_{a} p^{k)}+Y^{\alpha(i j}(x, y) \partial_{\alpha} p^{k)}+Z^{(i j k)}{ }_{l}(x, y) p^{l}
\end{aligned}
$$

where $X, Y$ and $Z$ are tensors of homogeneous polynomials of appropriate degree. For any particular choice of polynomial deformations $q^{i}, r^{j}$ and $s^{k}, \kappa_{q r s}$ is the corresponding Yukawa coupling.

Given our choice of defining polynomials $\vec{p}=(f, g, h)$ in $(2.2)$, it is straightforward to determine that

$$
Q(x, y)=Q x^{1} x^{2} x^{3} x^{4} y^{1} y^{2} y^{3} y^{4},
$$

where $Q$ is some uninteresting overall constant (only the relative ratios of Yukawa couplings have physical meaning).

More generally, for other (smooth) choices of the defining polynomials, i.e., different members of the Tian-Yau family of manifolds,

$$
Q(x, y)=Q \operatorname{det}\left[f^{\prime \prime}(x)\right] \operatorname{det}\left[g^{\prime \prime}(y)\right],
$$


where the double prime denotes second partial derivative with respect to the arguments. As $h^{\prime \prime}(x, y)$ is a matrix of constants, det $\left[h^{\prime \prime}\right]$ has been absorbed in the numerical factor $Q$.

On writing (6.2) out, we have

$$
\begin{aligned}
Q(x, y) & =Q d_{a b c d} x^{a} x^{b} x^{c} x^{d} d_{\alpha \beta \gamma \delta} y^{\alpha} y^{\beta} y^{\gamma} y^{\delta}, \\
d_{a b c d} & =\varepsilon^{e f g h} \varepsilon^{i j k l} f_{a e j} f_{b f j} f_{c g k} f_{d h l}, \\
d_{\alpha \beta \gamma \delta} & =\varepsilon^{\varepsilon \theta \kappa \lambda} \varepsilon^{\mu \nu \rho \sigma} g_{\alpha \varepsilon \mu} g_{\beta \theta \nu} g_{\gamma \kappa \rho} g_{\delta \lambda \sigma},
\end{aligned}
$$

where again we buried all uninteresting numerical factors into the prefactor $Q$. It is straightforward to verify that

$$
d_{a b c d}=d_{(a b c d)}= \begin{cases}1 & a, b, c, d \text { are all different; } \\ 0 & \text { otherwise } .\end{cases}
$$

The analogous is true of $d_{\alpha \beta \gamma \delta}$.

Let us write

$$
q^{1}=\varphi_{a b c}^{(q)} x^{a} x^{b} x^{c}, \quad r^{3}=\varphi^{(r)}{ }_{a \beta} x^{a} y^{\beta}, \quad s^{2}=\varphi^{(s)}{ }_{\alpha \beta \gamma} y^{\alpha} y^{\beta} y^{\gamma}
$$

and we can choose these tensors $\varphi$ among those listed in Table 2.

It is now not hard to see that the Yukawa coupling $\kappa_{q r s}$ in (6.1) can be rewritten as the contraction

$$
\kappa_{q r s}=d^{a b c d} d^{\alpha \beta \gamma \delta} \varphi^{(q)}{ }_{a b c} \varphi^{(r)}{ }_{d \delta} \varphi^{(s)}{ }_{\alpha \beta \gamma},
$$

where we have defined

$$
d^{a b c d}=d^{(a b c d)}= \begin{cases}1 & a, b, c, d \text { are all different; } \\ 0 & \text { otherwise }\end{cases}
$$

As a matter of fact, Eq. (6.6) is the only possible non-vanishing trace of a triple product of $\varphi$ 's from Table 2 that one can obtain using the available tensors $\left(\delta_{a}^{b}, \varepsilon_{a b c d}, \varepsilon^{a b c d}, \delta_{\alpha}^{\beta}, \varepsilon_{\alpha \beta \chi \delta}, \varepsilon^{\alpha \beta \chi \delta}, f_{a b c}, g_{\alpha \beta \gamma}\right.$ and $\left.h_{a \beta}\right)$.

Of course, in view of the relations (3.3) generated by the mappings in Table 2, one has to check if a particular triple product with "wrong" indices for contraction with $d^{a b c d} d^{\alpha \beta \gamma \delta}$ has no equivalent form in which the contraction can be performed.

In a way, this result was expected. The Yukawa coupling had to be a scalar quantity, obtained from the triple product of the representative tensors from Table 2 . To obtain a scalar quantity, the indices had to be contracted and, given that $\phi^{(q)} \phi^{(r)} \phi^{(s)}$ has only lower $\mathbb{P}^{3} \times \mathbb{P}^{3}$-indices, this is possible only with the tensors dual to $d_{a b c d}$ and $d_{\alpha \beta \gamma \delta}$ which, in turn, are defined in (6.4) and (6.5), respectively.

The tensor algebra on $\mathbb{P}^{3}=U(4) /[U(1) \times U(3)]$ consists of tensors with covariant and contravariant indices; these we can symmetrize and antisymmetrize and one can take traces with the $U(4)$-invariant tensor, the Kronecker $\delta_{a}^{b}$. Furthermore, as discussed in Appendix B, we can also factorize $\varepsilon^{a b c d}$ and $\varepsilon_{a b c d}$ even though they are not $U$ (4)-invariant; they transform only by a multiplicative $U(1) \subset U(4)$ phase. Once we pass to the hypersurface $f(x)=0$ in $\mathbb{P}_{x}^{3}$, the tensor algebra is endowed with a new "invariant" tensor, $f_{a b c}$, and we may use it to contract indices. The analogous is true of the hypersurface $g(y)=0$ in $\mathbb{P}_{y}^{3}$. Indeed, ' $d_{a b c d}$ and 
$d_{\alpha \beta \gamma \delta}$ may simply be viewed as an abbreviation for the more bewildering expressions (6.4) and (6.5).

It would follow that the algorithm [11] for computing the $27^{3}$ Yukawa couplings is correctly generalized to arbitrary Calabi-Yau complete intersections by the formula

$$
\kappa_{q r s}=\operatorname{Tr}_{f}, \ldots\left[\varphi^{(q)} \varphi^{(r)} \varphi^{(s)}\right]
$$

where $\mathbf{T r}_{f}, \ldots$ stands for obtaining the trace with the tensors associated to the defining equations, the Kronecker $\delta$ - and the Levi-Civita $\varepsilon$-symbols. This is examined further in ref. [5].

\section{The $27^{* 3}$ Yukawa Couplings}

The representatives for $H^{1}\left(\mathscr{M}, \mathscr{T}_{\mathscr{M}}^{*}\right)$, in Table 1 resemble qualitatively the basis of ref. [12]. The six symmetric, off-diagonal tensors $\phi^{(a b)}$ account for the six exceptional divisors $E_{1}^{x}, \ldots E_{6}^{x}$ and similarly for the $y$-part; the Kähler forms $J_{x}$ and $J_{y}$ occur both in our parametrization and in that of ref. [12].

Indeed, using the discrete symmetries of the manifold, the correspondence between our tensors and the exceptional divisors can be made precise and is given in Table 9; for each tensor, the corresponding linear combination is unique; no other combination has the same symmetry properties.

The $27^{* 3}$ Yukawa couplings were computed in ref. [12] using the exceptional divisors. Clearly, given the correspondence in Table 9 , we can translate this into the tensorial notation, obtaining the couplings in Table 10. Actually, owing to the very high degree of symmetry of $\mathscr{M}$, most of the $27^{* 3}$ couplings can be determined through symmetry considerations. Note that we may use all of the phase symmetries (5.2)-(5.4). Also, viewing the manifold $\mathscr{M}$ as a hypersurface in the product of

Table 8. Matter couplings stemming from $27^{3}$

\begin{tabular}{|c|c|c|}
\hline Value & Couplings on $\mathscr{M}_{1}$ & Couplings on $\mathscr{M}_{2}$ \\
\hline$-3 \kappa$ & $\left(q_{7} Q_{7} \lambda_{7}\right),\left(q_{1} Q_{1} \lambda_{7}\right)$ & $\left(q_{7} Q_{7} \lambda_{7}\right),\left(\lambda_{1} \lambda_{3} \lambda_{7}\right)$ \\
\hline$-2 \kappa$ & $\begin{array}{l}\left(q_{5} Q_{5} \lambda_{6}\right),\left(q_{2} Q_{2} \lambda_{6}\right),\left(\lambda_{1} \lambda_{3} \lambda_{5}\right) \\
\left(q_{1} q_{2} q_{3}\right),\left(q_{2} Q_{4} \lambda_{4}\right),\left(q_{2} Q_{5} \lambda_{3}\right) \\
\left(Q_{1} Q_{2} Q_{3}\right),\left(q_{6} Q_{1} \lambda_{4}\right),\left(q_{7} Q_{1} \lambda_{3}\right) \\
\left(q_{4} Q_{2} \lambda_{2}\right),\left(q_{1} Q_{6} \lambda_{4}\right),\left(\lambda_{2} \lambda_{3} \lambda_{8}\right) \\
\left(q_{5} Q_{2} \lambda_{1}\right),\left(q_{1} Q_{7} \lambda_{1}\right),\left(\lambda_{1} \lambda_{4} \lambda_{9}\right)\end{array}$ & $\begin{array}{l}\left(q_{5} Q_{5} \lambda_{6}\right),\left(q_{1} Q_{1} \lambda_{6}\right),\left(q_{2} Q_{2} \lambda_{5}\right) \\
\left(q_{3} Q_{1} \lambda_{3}\right),\left(q_{4} Q_{1} \lambda_{4}\right),\left(Q_{1} Q_{2} Q_{5}\right), \\
\left(q_{1} Q_{3} \lambda_{1}\right),\left(\lambda_{1} \lambda_{4} \lambda_{8}\right),\left(q_{7} Q_{2} \lambda_{1}\right) \\
\left(q_{1} Q_{4} \lambda_{2}\right),\left(\lambda_{3} \lambda_{4} \lambda_{9}\right),\left(q_{6} Q_{2} \lambda_{2}\right) \\
\left(q_{1} q_{2} q_{5}\right),\left(q_{2} Q_{7} \lambda_{3}\right),\left(q_{2} Q_{6} \lambda_{4}\right)\end{array}$ \\
\hline$-\kappa$ & $\begin{array}{l}\left(q_{3} Q_{5} \lambda_{2}\right),\left(q_{2} q_{6} q_{7}\right),\left(q_{4} Q_{1} \lambda_{8}\right) \\
\left(q_{3} Q_{7} \lambda_{4}\right),\left(q_{6} Q_{2} \lambda_{9}\right),\left(q_{1} q_{4} q_{5}\right) \\
\left(Q_{1} Q_{4} Q_{5}\right),\left(q_{7} Q_{3} \lambda_{2}\right),\left(q_{2} Q_{6} \lambda_{8}\right) \\
\left(q_{1} Q_{4} \lambda_{9}\right),\left(q_{5} Q_{3} \lambda_{4}\right),\left(Q_{2} Q_{6} Q_{7}\right)\end{array}$ & $\begin{array}{l}\left(q_{3} Q_{5} \lambda_{2}\right),\left(q_{7} Q_{1} \lambda_{8}\right),\left(q_{6} Q_{4} \lambda_{1}\right), \\
\left(q_{3} Q_{7} \lambda_{4}\right),\left(q_{1} Q_{6} \lambda_{8}\right),\left(q_{5} Q_{4} \lambda_{3}\right), \\
\left(q_{4} Q_{5} \lambda_{1}\right),\left(q_{7} Q_{3} \lambda_{2}\right),\left(q_{6} Q_{1} \lambda_{9}\right), \\
\left(q_{4} Q_{6} \lambda_{3}\right),\left(q_{5} Q_{3} \lambda_{4}\right),\left(q_{1} Q_{7} \lambda_{9}\right)\end{array}$ \\
\hline$\kappa$ & $\begin{array}{l}\left(q_{3} q_{5} q_{7}\right),\left(q_{4} Q_{5} \lambda_{9}\right),\left(q_{6} Q_{7} \lambda_{8}\right) \\
\left(q_{5} Q_{4} \lambda_{8}\right),\left(Q_{3} Q_{5} Q_{7}\right),\left(q_{7} Q_{6} \lambda_{9}\right) \\
\left(q_{7} Q_{7} \lambda_{6}\right),\left(\lambda_{6} \lambda_{8} \lambda_{9}\right),\left(q_{3} Q_{3} \lambda_{5}\right) \\
\left(q_{4} Q_{4} \lambda_{5}\right),\left(q_{6} Q_{6} \lambda_{5}\right),\left(\lambda_{2} \lambda_{4} \lambda_{6}\right) \\
\left(q_{1} Q_{1} \lambda_{6}\right)\end{array}$ & $\begin{array}{l}\left(q_{3} q_{5} q_{7}\right),\left(Q_{4} Q_{5} Q_{6}\right),\left(q_{6} Q_{7} \lambda_{8}\right), \\
\left(q_{4} q_{5} q_{6}\right),\left(Q_{3} Q_{5} Q_{7}\right),\left(q_{7} Q_{6} \lambda_{9}\right), \\
\left(q_{7} Q_{7} \lambda_{6}\right),\left(q_{6} Q_{6} \lambda_{6}\right),\left(q_{3} Q_{3} \lambda_{5}\right), \\
\left(q_{4} Q_{4} \lambda_{5}\right),\left(\lambda_{5} \lambda_{8} \lambda_{9}\right),\left(\lambda_{2} \lambda_{4} \lambda_{6}\right), \\
\left(\lambda_{1} \lambda_{3} \lambda_{6}\right)\end{array}$ \\
\hline $3 \kappa$ & $\left(\lambda_{7} \lambda_{8} \lambda_{9}\right),\left(\lambda_{2} \lambda_{4} \lambda_{7}\right)$ & $\left(q_{6} Q_{6} \lambda_{7}\right),\left(\lambda_{2} \lambda_{4} \lambda_{7}\right)$ \\
\hline
\end{tabular}


Table 9. The tensor representatives in terms of the basis of ref. [12], related to the exceptional divisors; $\gamma \xi=\frac{1}{\sqrt{3}} e^{5 i \pi / 6}$ and $\varepsilon=\varepsilon^{1234}$ and $\underline{\varepsilon}=\varepsilon^{12 \underline{2} \underline{3}} \underline{4}$

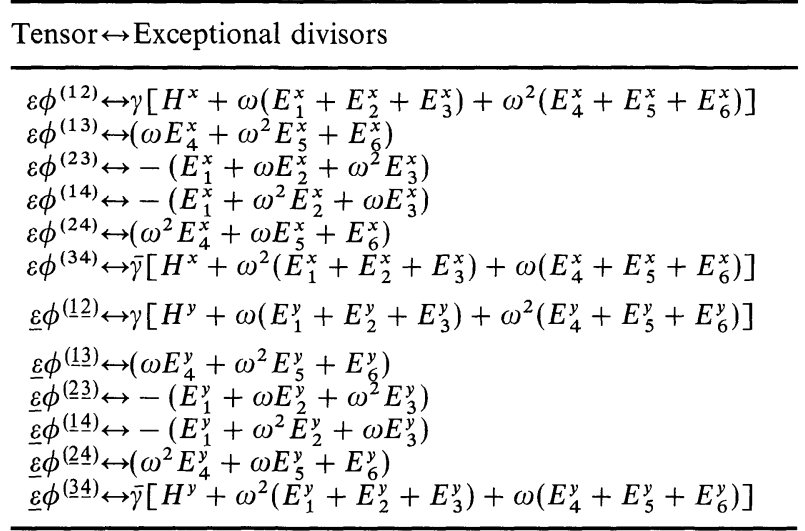

Table 10. Matter couplings stemming from $27 * 3$

\begin{tabular}{ll}
\hline Value & Couplings \\
\hline$\tilde{\kappa} / \sqrt{3}$ & $\left(\bar{\lambda}_{1} \bar{\lambda}_{3} \bar{\lambda}_{5}\right),\left(\bar{\lambda}_{1} \bar{\lambda}_{4} \bar{\lambda}_{6}\right),\left(\bar{\lambda}_{1} \bar{Q}_{1} \bar{q}_{3}\right),\left(\bar{\lambda}_{1} \bar{Q}_{3} \bar{q}_{1}\right),\left(\bar{\lambda}_{1} \bar{Q}_{2} \bar{q}_{4}\right),\left(\bar{\lambda}_{1} \bar{Q}_{4} \bar{q}_{2}\right)$ \\
$\tilde{\kappa} / \sqrt{2}$ & $-\left(\bar{\lambda}_{2} \bar{\lambda}_{3} \bar{\lambda}_{5}\right),\left(\bar{\lambda}_{2} \bar{\lambda}_{4} \bar{\lambda}_{6}\right),-\left(\bar{\lambda}_{2} \bar{Q}_{1} \bar{q}_{3}\right),\left(\bar{\lambda}_{2} \bar{Q}_{3} \bar{q}_{1}\right),-\left(\bar{\lambda}_{2} \bar{Q}_{2} \bar{q}_{4}\right),\left(\bar{\lambda}_{2} \bar{Q}_{4} \bar{q}_{2}\right)$ \\
$\tilde{\kappa} / \sqrt{3}$ & $\frac{1}{2}\left(\bar{\lambda}_{1} \bar{\lambda}_{1} \bar{\lambda}_{1}\right),-\left(\bar{\lambda}_{1} \bar{\lambda}_{2} \bar{\lambda}_{2}\right)$ \\
\hline
\end{tabular}

two cubic surfaces $\{f(x)=0\} \times\{g(y)=0\}$ and that we are wedging $(1,1)$-forms, it is straightforward that non-vanishing Yukawa couplings must be of the form

$$
\Xi^{2} \Upsilon \text { or } \Xi \Upsilon^{2}, \quad \Xi \in\left\{\xi, \varepsilon \phi^{(a b)}\right\}, \quad \Upsilon \in\left\{\eta, \underline{\varepsilon} \phi^{(\alpha \beta)}\right\} \text {. }
$$

Except for the relative ratio $\sqrt{2}: \sqrt{3}$, the results in Table 10 follow from the permutation symmetries $\sigma_{12}$ and $\sigma_{23}$.

Note that the fields in Table 6 were identified so that Table 10 is valid for both $\mathscr{M}_{1}$ and $\mathscr{M}_{2}$. Recalling Wall's classification theorem [27], one might suspect that the two manifolds and hence the two "low" energy models also are equivalent. Suffice it here to point out that Wall's classification theorem assumes simple connectedness, while both $\mathscr{M}_{1}$ and $\mathscr{M}_{2}$ have $\Pi_{1}=\mathbb{Z}_{3}$. In fact, it is precisely the differently warped action of this $\mathbb{Z}_{3}$ on the Yang-Mills charges of the matter fields which makes all the difference.

Having obtained all the matter fields in these two models in such a unifying way, we expect that the $27^{3}$ Yukawa coupling computation of the preceding section should somehow apply for the $27^{* 3}$ also. Consider one of the couplings on $\mathscr{M}_{2}$, expressed in terms of our representatives of Table 6:

$$
\left(\bar{\lambda}_{1} \bar{\lambda}_{3} \bar{\lambda}_{5}\right) \propto \frac{1}{\sqrt{3}}\left(J_{x}+J_{y}\right) \cdot\left(-\varepsilon^{a b c d} \phi^{(23)}\right) \cdot\left(-\varepsilon^{i j k l} \phi^{(14)}\right) .
$$


Tracing with $f_{a i 1} f_{b j 2} f_{c k 3} f_{d l 4}$, we obtain

Now, comparing with

$$
\left(\bar{\lambda}_{1} \bar{\lambda}_{3} \bar{\lambda}_{5}\right)=\frac{1}{\sqrt{3}} d_{1234} \phi^{(23)} \phi^{(14)} J_{y} .
$$

$$
\begin{aligned}
\left(\bar{\lambda}_{1} \bar{q}_{1} \bar{Q}_{3}\right) & =\frac{1}{\sqrt{2}}\left(J_{x}-J_{y}\right) \cdot\left(\varepsilon^{\alpha \beta \gamma \delta} \phi^{(1 \underline{3})} \cdot\left(\varepsilon^{\mu v \rho \sigma} \phi^{(\underline{2} 4)}\right) g_{\alpha \mu \underline{1}} g_{\beta v \underline{2}} g_{\gamma \rho \underline{3}} g_{\delta \sigma \underline{4}},\right. \\
& =\frac{1}{\sqrt{2}} d_{\underline{12} \underline{3} \underline{4}} \phi^{(\underline{1} 3)} \phi^{(2 \underline{4})} J_{x},
\end{aligned}
$$

the relative ratio $\sqrt{2}: \sqrt{3}$ follows since $d_{1234}$ and $d_{12 \underline{3} 4}$ have the same numerical value, labeled $\tilde{\kappa}$ in Table 10 . It is not hard to see that all couplings listed in Table 10 (and also the vanishing of all the others) are covered by a general formula such as (6.7), except for the last two. The ratio between these two and the others reflects the relative "size" of the Kähler form compared to the exceptional divisors in Table 9; this is an extra input for our computation.

\section{Other Yukawa Couplings}

We have seen that the same general formula as in Eq. (6.7) produces both the $27^{3}$ and the $27^{* 3}$ couplings. It is quite natural then to expect it to be completely general, producing all possible couplings - even beyond the renormalizable level. Unfortunately, for any coupling other than $27^{3}$ and $27^{* 3}$, we have no independent means of computation to check this. The rich symmetry structure of our paradigm will therefore serve as the only available check. It will be gratifying to learn that our general formula indeed passes this test. In fact, as is also generally the case with the $27^{3}$ and $27^{* 3}$ couplings; there will be relations and restrictions for which we could find no symmetry "reason."

We first list tables of couplings as restricted by the various symmetries of the model and will then comment on further results obtained using our general formula (6.7).

The reader will have noticed that there are many more values of unrelated couplings which involve the $E_{6}$ singlets, $s_{i}$ and $\tilde{s}_{i}$, in contrast to the case of the $27^{3}$ couplings. The relations among the various couplings in the $27^{3}$ case are provided by the equivalence relations (3.3). Similar relations do occur among candidate representatives for $s_{i}$ and $\tilde{s}_{i}$, but in a much lesser number, whence more of the coupling values remain unrelated at this stage.

Generalizing the arguments of Sect. 6.2, we know that a general trace formula such as (6.7) simply must be valid for all couplings. Once the matter fields are represented in terms of tensor-components from the $U_{x}(4) \times U_{y}(4)$ tensor algebra (projected in various ways by using the "defining" tensors $f_{a b c}, g_{\alpha \beta \gamma}$ and $h_{a \alpha}$ ), a physically measurable quantity simply cannot be anything else. The adventurous reader is invited to provide a more direct derivation, perhaps along the lines of ref. [11].

As a sample computation, compare the couplings $\left(\bar{\lambda}_{1} \bar{\lambda}_{3} \bar{\lambda}_{5}\right)$ on $\mathscr{M}_{2}$, from Table 10, and $j_{5}$ from Table 14:

$$
\begin{aligned}
& \left(\bar{\lambda}_{1} \bar{\lambda}_{3} \bar{\lambda}_{5}\right)=\operatorname{Tr}\left[\frac{1}{\sqrt{3}}\left(J_{x}+J_{y}\right)\left(-\varepsilon^{1234} \phi^{(23)}\right)\left(-\varepsilon^{1234} \phi^{(14)}\right)\right], \\
& \left(\lambda_{5} \bar{\lambda}_{5} \tilde{s}_{9}\right)=\operatorname{Tr}\left[\left(\phi_{44}\right)\left(-\varepsilon^{1234} \phi^{(14)}\right)\left(\varepsilon^{12 \underline{3}-\underline{4}} \phi^{[144]}\right)\right] .
\end{aligned}
$$


Firstly, the left-hand side of the latter of these expressions must be multiplied by $\varepsilon_{1234} \varepsilon_{1234}$ for the one $\Omega$ in Eqs. (5.9) and in view of the formula (2.15). To complete the trace in the first of these expressions, we must still multiply it by $f_{111} f_{222} f_{333} f_{444}$. The available indices can be contracted in precisely 1 way, so that

$$
\tilde{\kappa}=f_{111} f_{222} f_{333} f_{444} \text {. }
$$

To complete the trace in the second coupling above, we must multiply it with $h_{11}$. There are precisely 12 distinct and non-vanishing ways to contract the available indices, so that

$$
j_{5}=-12 h_{11} .
$$

The relative value, is therefore

$$
\left(\bar{\lambda}_{1} \bar{\lambda}_{3} \bar{\lambda}_{5}\right) /\left(\lambda_{5} \bar{\lambda}_{5} \tilde{s}_{9}\right)=\frac{-f_{111} f_{222} f_{333} f_{444}}{12 \sqrt{3} h_{11}},
$$

which in our case equals $-1 /(12 \sqrt{3})$. More generally, however, one can see that the relative combinatorial/normalization factors, such as $12 \sqrt{3}$ here, may improve on the structure in the pattern of Yukawa couplings.

Unfortunately, the exact value is however deceiving in part, since the various spacetime fields would have to be renormalized by the respective norm-factors, listed in Tables 5, 6 and 7 in the column "Norm." These factors are unknown, apart from the restrictions imposed by symmetries, and this was already used in labeling them.

An explicit evaluation of all relations among the various couplings listed in Tables $8,10,11,12,13$ and 14 is now straightforward. Given that the rather simple

\begin{tabular}{|c|c|c|c|}
\hline Value & Couplings & Value & Couplings \\
\hline $\begin{array}{c}\tilde{l}_{1} \\
\tilde{l}_{2} \\
\tilde{l}_{3} \\
\tilde{l}_{4} \\
\tilde{l}_{5} \\
\tilde{l}_{6} \\
\tilde{l}_{7} \\
\tilde{l}_{8} \\
\tilde{l}_{9} \\
\tilde{l}_{10} \\
\tilde{l}_{11} \\
\tilde{l}_{12} \\
\tilde{l}_{13} \\
\tilde{l}_{14} \\
\tilde{l}_{15} \\
\tilde{l}_{16} \\
\tilde{l}_{17} \\
\tilde{l}_{18}\end{array}$ & $\begin{array}{l}\left(s_{3} s_{2} \tilde{s}_{8}\right),-\left(s_{4} s_{2} \tilde{s}_{8}\right) \\
\left(s_{1} s_{6} \tilde{s}_{8}\right) \\
\left(\tilde{s}_{2} s_{5} s_{13}\right),-\left(\tilde{s}_{2} s_{5} s_{14}\right) \\
\left(\tilde{s}_{2} s_{6} s_{13}\right),-\left(\tilde{s}_{2} s_{6} s_{14}\right) \\
\left(s_{3} s_{5} \tilde{s}_{16}\right), 3\left(s_{4} s_{5} \tilde{s}_{16}\right) \\
\left(s_{4} s_{6} \tilde{s}_{16}\right),-\left(s_{3} s_{6} \tilde{s}_{16}\right) \\
\left(s_{8} s_{8} \tilde{s}_{16}\right) \\
\left(\tilde{s}_{5} s_{10} s_{10}\right) \\
\left(s_{10} s_{10} \tilde{s}_{15}\right) \\
\left(s_{10} s_{12} s_{12}\right) \\
\left(\tilde{s}_{8} s_{13} s_{13}\right),-\left(\tilde{s}_{8} s_{14} s_{14}\right) \\
\left(s_{13} s_{16} s_{16}\right),-\left(s_{14} s_{16} s_{16}\right) \\
\left(\tilde{s}_{1} s_{17} s_{17}\right) \\
\left(s_{3} s_{8} \tilde{s}_{12}\right), 3\left(s_{4} s_{8} \tilde{s}_{12}\right) \\
\left(\tilde{s}_{3} s_{9} s_{11}\right),-\left(\tilde{s}_{4} s_{9} s_{11}\right) \\
\left(s_{3} s_{10} s_{17}\right) 3\left(s_{4} s_{10} s_{17}\right) \\
\left(s_{3} s_{15} \tilde{s}_{16}\right), 3\left(s_{4} s_{15} \tilde{s}_{16}\right) \\
\left(s_{3} s_{16} \tilde{s}_{17}\right), 3\left(s_{4} s_{16} \tilde{s}_{17}\right)\end{array}$ & $\begin{array}{l}\tilde{l}_{19} \\
\tilde{l}_{20} \\
\tilde{l}_{21} \\
\tilde{l}_{22} \\
\tilde{l}_{23} \\
\tilde{l}_{24} \\
\tilde{l}_{25} \\
\tilde{l}_{26} \\
\tilde{l}_{27} \\
\tilde{l}_{28} \\
\tilde{l}_{29} \\
\tilde{l}_{30} \\
\tilde{l}_{31} \\
\tilde{l}_{32} \\
\tilde{l}_{33} \\
\tilde{l}_{34} \\
\tilde{l}_{35} \\
\tilde{l}_{36}\end{array}$ & $\begin{array}{l}\left(\tilde{s}_{1} s_{7} s_{15}\right) \\
\left(\tilde{s}_{2} s_{8} s_{11}\right) \\
\left(s_{2} s_{9} s_{15}\right) \\
\left(s_{2} s_{11} \tilde{s}_{12}\right) \\
\left(s_{1} s_{10} \tilde{s}_{12}\right) \\
\left(s_{4} \tilde{s}_{12} s_{13}\right),\left(s_{1} \tilde{s}_{12} s_{14}\right) \\
\left(s_{6} s_{8} \tilde{s}_{9}\right) \\
\left(s_{6} \tilde{s}_{8} s_{17}\right) \\
\left(s_{6} \tilde{s}_{12} s_{15}\right) \\
\left(\tilde{s}_{6} s_{11} s_{16}\right) \\
\left(s_{7} \tilde{s}_{8} s_{13}\right),-\left(s_{7} \tilde{s}_{8} s_{14}\right) \\
\left(s_{7} s_{10} \tilde{s}_{11}\right) \\
\left(s_{7} s_{9} s_{12}\right) \\
\left(s_{7} \tilde{s}_{12} s_{17}\right) \\
\left(\tilde{s}_{8} s_{15} s_{17}\right) \\
\left(s_{9} s_{12} s_{13}\right),\left(s_{9} s_{12} s_{14}\right) \\
\left(s_{10} s_{11} s_{13}\right),\left(s_{10} s_{11} s_{14}\right) \\
\left(\tilde{s}_{12} s_{13} s_{17}\right),\left(\tilde{s}_{12} s_{14} s_{17}\right)\end{array}$ \\
\hline
\end{tabular}

Table 11. Matter couplings stemming from $1^{3}$ on $\mathscr{M}_{1}$. This only represents half of the nonvanishing couplings. The other half is obtained by using $\sigma_{x y}$ 
Table 12. Matter couplings stemming from $\mathbf{1}^{3}$ on $\mathscr{M}_{2}$. The couplings in the last row vanish only to first order in $\sigma$-perturbation theory. Note also that we listed only half of the non-vanishing couplings; the other half is obtained by using $\sigma_{x y}$

\begin{tabular}{|c|c|c|c|}
\hline Value & Couplings & Value & Couplings \\
\hline $\begin{array}{l}l_{1} \\
l_{2} \\
l_{3} \\
l_{4} \\
l_{5} \\
l_{6} \\
l_{7} \\
l_{8} \\
l_{9} \\
l_{10} \\
l_{11} \\
l_{12} \\
l_{13} \\
l_{14} \\
l_{15} \\
0\left(L_{1}\right)\end{array}$ & $\begin{array}{l}\left(s_{3} s_{6} \tilde{s}_{10}\right),-\left(s_{4} s_{5} \tilde{s}_{10}\right) \\
\left(s_{1} s_{6} s_{7}\right),\left(s_{2} s_{5} s_{8}\right) \\
\left(s_{1} s_{4} s_{7}\right),\left(s_{2} s_{3} s_{8}\right) \\
\left(s_{1} s_{5} \tilde{s}_{17}\right),\left(s_{2} s_{6} \tilde{s}_{17}\right) \\
\left(s_{1} \tilde{s}_{6} s_{17}\right),\left(s_{2} \tilde{s}_{5} s_{17}\right) \\
\left(s_{1} s_{3} \tilde{s}_{17}\right),\left(s_{2} s_{4} \tilde{s}_{17}\right) \\
\left(s_{1} s_{4} s_{17}\right),\left(s_{2} \tilde{s}_{3} s_{17}\right) \\
\left(s_{10} s_{10} \tilde{s}_{17}\right) \\
\left(s_{7} s_{7} \tilde{s}_{16}\right)-\left(s_{8} s_{8} \bar{s}_{15}\right) \\
\left(s_{7} s_{8} \tilde{s}_{13}\right),-\left(s_{7} s_{8} \tilde{s}_{14}\right) \\
\left(s_{8} s_{11} s_{11}\right),\left(s_{7} s_{12} s_{12}\right) \\
\left(s_{11} s_{12} \tilde{s}_{17}\right) \\
\left(\tilde{s}_{10} s_{13} s_{13}\right),-\left(\tilde{s}_{10} s_{14} s_{14}\right) \\
\left(\tilde{s}_{12} s_{16} s_{16}\right),-\left(\tilde{s}_{11} s_{15} s_{15}\right) \\
\left(s_{13} s_{17} s_{17}\right),-\left(s_{14} s_{17} s_{17}\right) \\
\left(\tilde{s}_{3} s_{7} s_{7}\right),\left(\tilde{s}_{4} s_{8} s_{8}\right)\end{array}$ & $\begin{array}{l}l_{16} \\
l_{17} \\
l_{18} \\
l_{19} \\
l_{20} \\
l_{21} \\
l_{22} \\
l_{23} \\
l_{24} \\
l_{25} \\
l_{26} \\
l_{27} \\
l_{28} \\
l_{29} \\
l_{30}\end{array}$ & $\begin{array}{l}\left(s_{1} s_{8} s_{9}\right),-\left(s_{2} s_{7} s_{9}\right) \\
\left(s_{1} s_{10} \tilde{s}_{12}\right),\left(s_{2} s_{10} \tilde{s}_{11}\right) \\
\left(s_{1} s_{7} s_{15}\right),-\left(s_{2} s_{8} s_{16}\right) \\
\left(s_{1} s_{8} s_{13}\right),-\left(s_{2} s_{7} s_{14}\right) \\
\left(s_{1} s_{8} s_{14}\right),-\left(s_{2} s_{7} s_{13}\right) \\
\left(s_{1} s_{16} s_{17}\right),-\left(s_{2} s_{15} \tilde{s}_{17}\right) \\
\left(s_{3} s_{9} \tilde{s}_{12}\right),\left(s_{5} s_{9} \tilde{s}_{11}\right) \\
\left(s_{4} s_{9} \tilde{s}_{12}\right),\left(s_{6} s_{9} \tilde{s}_{11}\right) \\
\left(s_{3} \tilde{s}_{10} s_{16}\right),\left(s_{5} \tilde{s}_{10} s_{15}\right) \\
\left(\tilde{s}_{4} \tilde{s}_{10} s_{16}\right),\left(s_{6} \tilde{s}_{10} s_{15}\right) \\
\left(s_{3} \tilde{s}_{11} s_{15}\right),\left(s_{5} \tilde{s}_{12} s_{16}\right) \\
\left(s_{4} \tilde{s}_{11} s_{15}\right),\left(s_{6} \tilde{s}_{12} s_{16}\right) \\
\left(s_{9} \tilde{s}_{10} s_{13}\right),-\left(s_{9} \tilde{s}_{10} s_{14}\right) \\
\left(s_{9} \tilde{s}_{11} s_{16}\right),-\left(s_{9} \tilde{s}_{12} s_{15}\right) \\
\left(s_{7} s_{10} s_{11}\right),\left(s_{8} s_{10} s_{12}\right)\end{array}$ \\
\hline
\end{tabular}

Table 13. Matter couplings stemming from $27 \cdot 27 * \cdot 1$ on $\mathscr{M}_{1}$

\begin{tabular}{llll}
\hline Value & Couplings & Value & Couplings \\
\hline$\tilde{j_{1}}$ & $\left(\lambda_{1} \bar{\lambda}_{2} s_{9}\right),-\left(\lambda_{3} \bar{\lambda}_{2} \tilde{s}_{9}\right)$ & $\tilde{j_{6}}$ & $\left(\lambda_{6} \bar{\lambda}_{3} \tilde{s}_{8}\right),\left(\lambda_{6} \bar{\lambda}_{6} s_{8}\right)$ \\
$\tilde{j_{2}}$ & $\left(\lambda_{1} \bar{\lambda}_{3} \tilde{s}_{12}\right),\left(\lambda_{3} \bar{\lambda}_{6} s_{12}\right)$ & $\tilde{j_{7}}$ & $\left(\lambda_{6} \bar{\lambda}_{4} s_{16}\right)$ \\
$\tilde{j_{3}}$ & $\left(\lambda_{2} \bar{\lambda}_{2} s_{10}\right),-\left(\lambda_{4} \bar{\lambda}_{2} \tilde{s}_{10}\right)$ & $\tilde{j_{8}}$ & $\left(\lambda_{7} \bar{\lambda}_{3} \tilde{s}_{8}\right),\left(\lambda_{7} \bar{\lambda}_{6} s_{8}\right)$ \\
$\tilde{j_{4}}$ & $\left(\lambda_{5} \bar{\lambda}_{4} s_{7}\right),\left(\lambda_{5} \bar{\lambda}_{5} \tilde{s}_{7}\right)$ & $\tilde{j_{9}}$ & $\left(\lambda_{7} \bar{\lambda}_{4} s_{16}\right),\left(\lambda_{7} \bar{\lambda}_{5} \tilde{s}_{16}\right)$ \\
& $\left(\lambda_{5} \bar{\lambda}_{4} s_{13}\right),-\left(\lambda_{5} \bar{\lambda}_{4} s_{14}\right)$, & $\tilde{j}_{10}$ & $\left(\lambda_{8} \bar{\lambda}_{4} s_{12}\right),\left(\lambda_{9} \bar{\lambda}_{5} \tilde{s}_{12}\right)$ \\
$\tilde{j_{5}}$ & $\left(\lambda_{5} \bar{\lambda}_{5} \tilde{s}_{13}\right),-\left(\lambda_{5} \bar{\lambda}_{5} \tilde{s}_{14}\right)$ & & $\left(q_{5} \bar{q}_{2} s_{7}\right),\left(q_{7} \bar{q}_{3} \tilde{s}_{7}\right)$, \\
$\tilde{k_{1}}$ & $\left(q_{1} \bar{q}_{1} s_{12}\right),\left(q_{2} \bar{q}_{4} \tilde{s}_{12}\right)$, & $\tilde{k}_{6}$ & $\left(Q_{5} \bar{Q}_{2} \tilde{s}_{7}\right),\left(Q_{7} \bar{Q}_{3} s_{7}\right)$ \\
& $\left(Q_{1} \bar{Q}_{1} \tilde{s}_{12}\right),\left(Q_{2} \bar{Q}_{4} s_{12}\right)$ & & $\left(q_{5} \bar{q}_{2} s_{14}\right),\left(q_{7} \bar{q}_{3} \tilde{s}_{13}\right)$, \\
$\tilde{k_{2}}$ & $-\left(q_{1} \bar{q}_{3} \tilde{s}_{15}\right),\left(q_{2} \bar{q}_{2} s_{15}\right)$, & $\tilde{k}_{7}$ & $\left(Q_{5} \bar{Q}_{2} \tilde{s}_{14}\right),\left(Q_{7} \bar{Q}_{3} s_{13}\right)$ \\
& $-\left(Q_{1} \bar{Q}_{3} s_{15}\right),\left(Q_{2} \bar{Q}_{2} \tilde{s}_{15}\right)$ & & $\left(q_{5} \bar{q}_{2} s_{13}\right),\left(q_{7} \bar{q}_{3} \tilde{s}_{14}\right)$, \\
$\tilde{k_{3}}$ & $-\left(q_{4} \bar{q}_{1} s_{16}\right),\left(q_{6} \bar{q}_{4} \tilde{s}_{16}\right)$, & $\tilde{k}_{8}$ & $\left(Q_{5} \bar{Q}_{2} \tilde{s}_{13}\right),\left(Q_{7} \bar{Q}_{3} s_{14}\right)$ \\
& $-\left(Q_{4} \bar{Q}_{1} \tilde{s}_{16}\right),\left(Q_{6} \bar{Q}_{4} s_{16}\right)$ & & $\left(q_{5} \bar{q}_{4} \tilde{s}_{8}\right),\left(q_{7} \bar{q}_{1} s_{8}\right)$, \\
$\tilde{k}_{4}$ & $\left(q_{4} \bar{q}_{2} s_{12}\right),\left(q_{6} \bar{q}_{3} \tilde{s}_{12}\right)$, & & $\left(Q_{5} \bar{Q}_{4} s_{8}\right),\left(Q_{7} \bar{Q}_{1} \tilde{s}_{8}\right)$, \\
& $\left(Q_{4} \bar{Q}_{2} \tilde{s}_{12}\right),\left(Q_{6} \bar{Q}_{3} s_{12}\right)$ & & \\
$\tilde{k}_{5}$ & $\left(q_{4} \bar{q}_{3} \tilde{s}_{8}\right),\left(q_{6} \bar{q}_{2} s_{8}\right)$, & & \\
& $\left(Q_{4} \bar{Q}_{3} s_{8}\right),\left(Q_{6} \bar{Q}_{2} \tilde{s}_{8}\right)$ & & \\
\hline
\end{tabular}


Table 14. Matter couplings stemming from $27 \cdot 27 * \cdot 1$ on $\mathscr{M}_{2}$. The couplings in the last row vanish only to first order in $\sigma$-perturbation theory

\begin{tabular}{|c|c|c|c|}
\hline Value & Couplings & Value & Couplings \\
\hline$j_{1}$ & $\begin{array}{l}\left(\lambda_{1} \bar{\lambda}_{1} s_{8}\right),\left(\lambda_{2} \bar{\lambda}_{1} s_{7}\right) \\
\left(\lambda_{3} \bar{\lambda}_{1} \tilde{s}_{8}\right),\left(\lambda_{4} \bar{\lambda}_{1} \tilde{s}_{7}\right)\end{array}$ & $j_{6}$ & $\begin{array}{l}\left(\lambda_{5} \bar{\lambda}_{5} \tilde{s}_{13}\right),\left(\lambda_{5} \bar{\lambda}_{5} \tilde{s}_{14}\right), \\
\left(\lambda_{5} \bar{\lambda}_{6} s_{13}\right),\left(\lambda_{5} \bar{\lambda}_{6} s_{14}\right)\end{array}$ \\
\hline$j_{2}$ & $\begin{array}{l}\left(\lambda_{1} \bar{\lambda}_{2} s_{8}\right),\left(\lambda_{2} \bar{\lambda}_{2} s_{7}\right) \\
-\left(\lambda_{3} \bar{\lambda}_{2} \tilde{s}_{8}\right),-\left(\lambda_{4} \bar{\lambda}_{2} \tilde{s}_{7}\right)\end{array}$ & $j_{7}$ & $\left(\lambda_{6} \bar{\lambda}_{3} \tilde{s}_{10}\right),\left(\lambda_{6} \bar{\lambda}_{4} s_{10}\right)$ \\
\hline$j_{3}$ & $\begin{array}{l}\left(\lambda_{1} \bar{\lambda}_{4} s_{16}\right),\left(\lambda_{2} \bar{\lambda}_{4} s_{15}\right) \\
\left(\lambda_{3} \bar{\lambda}_{3} \tilde{s}_{16}\right),\left(\lambda_{4} \bar{\lambda}_{3} \tilde{s}_{15}\right)\end{array}$ & $j_{8}$ & $\left(\lambda_{7} \bar{\lambda}_{5} \tilde{s}_{17}\right),\left(\lambda_{7} \bar{\lambda}_{6} s_{17}\right)$ \\
\hline$j_{4}$ & $\begin{array}{l}\left(\lambda_{1} \bar{\lambda}_{5} \tilde{s}_{12}\right),\left(\lambda_{2} \bar{\lambda}_{5} \tilde{s}_{11}\right) \\
\left(\lambda_{3} \bar{\lambda}_{6} s_{12}\right),\left(\lambda_{4} \bar{\lambda}_{6} s_{11}\right)\end{array}$ & $j_{9}$ & $\begin{array}{l}\left(\lambda_{8} \bar{\lambda}_{3} \tilde{s}_{12}\right),\left(\lambda_{8} \bar{\lambda}_{4} s_{11}\right), \\
\left(\lambda_{9} \bar{\lambda}_{3} \tilde{s}_{11}\right),\left(\lambda_{9} \bar{\lambda}_{4} s_{12}\right)\end{array}$ \\
\hline$j_{5}$ & $\left(\lambda_{5} \bar{\lambda}_{5} \tilde{s}_{9}\right),\left(\lambda_{5} \bar{\lambda}_{6} s_{9}\right)$ & $j_{10}$ & $\begin{array}{l}\left(\lambda_{8} \bar{\lambda}_{5} s_{8}\right),\left(\lambda_{8} \bar{\lambda}_{6} \tilde{s}_{7}\right) \\
\left(\lambda_{9} \bar{\lambda}_{5} s_{7}\right),\left(\lambda_{9} \bar{\lambda}_{6} \tilde{s}_{8}\right)\end{array}$ \\
\hline$k_{1}$ & $\begin{array}{l}\left(q_{1} \bar{q}_{3} \tilde{s}_{16}\right),\left(q_{1} \bar{q}_{4} \tilde{s}_{15}\right) \\
\left(Q_{1} \bar{Q}_{3} s_{16}\right),\left(Q_{1} \bar{Q}_{4} s_{15}\right)\end{array}$ & $k_{7}$ & $\begin{array}{l}\left(q_{6} \bar{q}_{3} \tilde{s}_{13}\right),\left(q_{7} \bar{q}_{4} \tilde{s}_{14}\right), \\
\left(Q_{6} \bar{Q}_{3} s_{13}\right),\left(Q_{7} \bar{Q}_{4} s_{14}\right)\end{array}$ \\
\hline$k_{2}$ & $\begin{array}{l}\left(q_{1} \bar{q}_{1} s_{12}\right),\left(q_{1} \bar{q}_{2}, s_{11}\right) \\
\left(Q_{1} \bar{Q}_{1} \tilde{s}_{12}\right),\left(Q_{1} \bar{Q}_{2} \tilde{s}_{11}\right)\end{array}$ & $k_{8}$ & $\begin{array}{l}\left(q_{6} \bar{q}_{3} \tilde{s}_{14}\right),\left(q_{7} \bar{q}_{4} \tilde{s}_{13}\right), \\
\left(Q_{6} \bar{Q}_{3} s_{14}\right),\left(Q_{7} \bar{Q}_{4} s_{13}\right)\end{array}$ \\
\hline$k_{3}$ & $\begin{array}{l}\left(q_{2} \bar{q}_{4} \tilde{s}_{12}\right),\left(q_{2} \bar{q}_{3} \tilde{s}_{11}\right) \\
\left(Q_{2} \bar{Q}_{4} s_{12}\right),\left(Q_{2} \bar{Q}_{3} s_{11}\right)\end{array}$ & $k_{9}$ & $\begin{array}{l}\left(q_{6} \bar{q}_{2} s_{12}\right),\left(q_{7} \bar{q}_{1} s_{11}\right), \\
\left(Q_{6} \bar{Q}_{2} \tilde{s}_{12}\right),\left(Q_{7} \bar{Q}_{1} \tilde{s}_{11}\right)\end{array}$ \\
\hline$k_{4}$ & $\begin{array}{l}\left(q_{3} \bar{q}_{1} s_{17}\right),-\left(q_{4} \bar{q}_{2} s_{17}\right) \\
\left(Q_{3} \bar{Q}_{1} \tilde{s}_{17}\right),-\left(Q_{4} \bar{Q}_{2} \tilde{s}_{17}\right)\end{array}$ & $k_{10}$ & $\begin{array}{l}\left(q_{6} \bar{q}_{1} s_{10}\right),\left(q_{7} \bar{q}_{2} s_{10}\right), \\
\left(Q_{6} \bar{Q}_{1} \tilde{s}_{10}\right),\left(Q_{7} \bar{Q}_{2} \tilde{s}_{10}\right)\end{array}$ \\
\hline$k_{5}$ & $\begin{array}{l}\left(q_{3} \bar{q}_{3} \tilde{s}_{10}\right),\left(q_{4} \bar{q}_{4} \tilde{s}_{10}\right) \\
\left(Q_{3} \bar{Q}_{3} s_{10}\right),\left(Q_{4} \bar{Q}_{4} s_{10}\right)\end{array}$ & $k_{11}$ & $\begin{array}{l}\left(q_{6} \bar{q}_{3} \tilde{s}_{9}\right),\left(q_{7} \bar{q}_{4} \tilde{s}_{9}\right) \\
\left(Q_{6} \bar{Q}_{3} s_{9}\right),\left(Q_{7} \bar{Q}_{4} s_{9}\right)\end{array}$ \\
\hline$k_{6}$ & $\begin{array}{l}\left(q_{3} \bar{q}_{4} \tilde{s}_{11}\right),\left(q_{4} \bar{q}_{3} \tilde{s}_{12}\right) \\
\left(Q_{3} \bar{Q}_{4} s_{11}\right),\left(Q_{4} \bar{Q}_{3} s_{12}\right)\end{array}$ & & \\
\hline $0\left(K_{1}\right)$ & $\begin{array}{l}\left(q_{6} \bar{q}_{4} \tilde{s}_{5}\right),-\left(q_{7} \bar{q}_{3} \bar{s}_{6}\right) \\
\left(Q_{6} \bar{Q}_{4} s_{5}\right),-\left(Q_{7} \bar{Q}_{3} s_{6}\right)\end{array}$ & $0\left(K_{2}\right)$ & $\begin{array}{l}\left(q_{6} \bar{q}_{4} \tilde{s}_{16}\right),\left(q_{7} \bar{q}_{3} \tilde{s}_{15}\right) \\
\left(Q_{6} \bar{Q}_{4} s_{16}\right),\left(Q_{7} \bar{Q}_{3} s_{15}\right)\end{array}$ \\
\hline
\end{tabular}

choice (2.2) probably does not lead to reasonable phenomenology, we shall not evaluate these ratios here.

\section{Concluding Remarks}

To summarize briefly, we have shown on an explicit 3-generation Calabi-Yau compactification model that all of the light matter superfields can be parametrized by a single method. The representatives turn out to be various $U(4) \times U(4)$ tensor components, which allows us to generalize the $27^{3}$ Yukawa coupling formula of ref. [11] to the complete matter sector. This general formula simply turns out to be a generalized trace of the product of field representatives in question, possibly multiplied with a number of "special" tensors which include the usual Kronecker $\delta$ 's, Levi-Civitta $\varepsilon$ 's and in particular the tensors defined through the defining equations. For example, our choice (2.2) defines

$$
f_{a b c}= \begin{cases}1 & a=b=c=1,2,3,4, \\ 0 & \text { otherwise }\end{cases}
$$




$$
\begin{aligned}
& g_{\alpha \beta \gamma}= \begin{cases}1 & \alpha=\beta=\gamma=1,2,3,4, \\
0 & \text { otherwise } ;\end{cases} \\
& h_{\alpha \beta}= \begin{cases}1 & a=\beta=1,2,3, \\
0 & \text { otherwise } .\end{cases}
\end{aligned}
$$

The tensor algebra on the Calabi-Yau manifold is thus seen to differ from the tensor algebra on its embedding space in that there are "new" tensors, $f_{a b c}, g_{\alpha \beta \gamma}$ and $h_{\alpha \beta}$, with which one can take traces, project components, etc.

The harderst part in employing the presented technique is undoubtedly the application of spectral sequences in obtaining the tensorial representatives in Tables 5, 6 and 7. It would appear that the entire calculation would have to be redone for each different choice of defining equations. One would appreciate a computer-mechanized version of this task.

However, if one is interested merely in the transformation properties with respect to the various symmetries, the present tables can be used straightforwardly. This follows since the defining equations can be varied continuously, while the "charges" with respect to the various symmetries cannot. We emphasize again that the number of $E_{6}$ singlet fields, in Table 7 however will vary but in a very simple way and depending only on $\operatorname{rank}\left[h_{a \beta}\right]$. The representatives 31.-49. and their $\mathbb{P}_{x}^{3} \leftrightarrow \mathbb{P}_{y}^{3}$ counterparts will vanish from the light matter spectrum if $\operatorname{rank}\left[h_{a \beta}\right]=4$ rather than 3 as was the case considered here.

This enables an easy correspondence with a number of models found in the literature $[6,9,21,22,23]$ through simple relabeling of coordinates and shifting Eqs. (2.2) by a few parameters. Treating these different parameters perturbatively, one may even use the representatives in Tables 5, 6 and 7 as they are, to the lowest order.

Finally, we remind the rader that little is known about the world sheet instantons effect on the $\mathbf{1}^{3}$ and $27 \cdot 27^{*} \cdot \mathbf{1}$ couplings or about the normalization factors, so that our results have to be taken with a grain of salt in these respects.

Acknowledgements. We happily thank Michael G. Eastwood for teaching us the Bott-Borel-Weil theorem and its application. P.B. was supported by the Fulbright Program, the Foundation Blanceflor Boncompagni-Ludovisi Née Bildt and a Robert A. Welch Predoctoral Fellowship. L.P. was supported by the Robert A. Welch Foundation and the NSF Grant PHY9009850. T.H. gratefully acknowledges the support by the Robert A. Welch Foundation and the NSF Grant PHY8605978 during the early part of this project and the DOE grant DE-FG02-88ER-25065 in the later period.

\section{Appendices}

Spectral sequences prove to be quite indispensable in our analysis but are still mystifying most of the physics audience. In the following appendices, we hope to provide at least a set of working rules and algorithms, in an accessible form sufficient to reproduce our present results. We refer to ref. $[3,4,16,17]$ and, ultimately, ref. [1] for more details. Several computations in full detail, which have been omitted from the main part of the text, are also appended - the undertaking reader may use them to strengthen his understanding. First of all, however, we prove that the Calabi-Yau spaces discussed here are indeed manifolds, i.e., smooth; this extends the results of ref. [23]. 


\section{A. Smoothness}

In this appendix, we prove that the two three-generation Calabi-Yau manifolds, $\mathscr{M} / \varpi_{1}$ and $\mathscr{M} / \varpi_{2}$, are indeed smooth.

A.1. The Covering Space Smoothness. We first prove that the system

$$
\begin{aligned}
& f(x)=\left(x^{1}\right)^{3}+\left(x^{2}\right)^{3}+\left(x^{3}\right)^{3}+\left(x^{4}\right)^{3}=0, \\
& g(x)=\left(y^{1}\right)^{3}+\left(y^{2}\right)^{3}+\left(y^{3}\right)^{3}+\left(y^{4}\right)^{3}=0, \\
& h(x, y)=x^{1} y^{1}+x^{2} y^{2}+\eta x^{3} y^{3}=0
\end{aligned}
$$

has a smooth space of solutions $\mathscr{M} \subset \mathbb{P}^{3} \times \mathbb{P}^{3}$ precisely for $\eta^{3} \neq 0,-1,-2$.

We first introduce some notation. Let

$$
z^{A}= \begin{cases}x^{a} & \text { for } A=a=1,2,3,4 \\ y^{\alpha} & \text { for } A=\alpha+4=5,6,7,8 .\end{cases}
$$

To verify smoothness, we need to check that the volume form of the normal bundle is non-vanishing wherever the polynomials vanish, i.e., on the space of solutions. This volume form is

$$
d V \equiv V_{A B C} d z^{A} \wedge d z^{B} \wedge d z^{C} \stackrel{\text { def }}{=} d f \wedge d g \wedge d h
$$

where the gradients of the defining polynomials are

$$
d f=d z^{A} \partial_{A} f, \quad d g=d z^{A} \partial_{A} g, \quad d h=d z^{A} \partial_{A} h, \quad \partial_{A}=\frac{\partial}{\partial z^{A}} .
$$

The following is a list of $12 \times 4$ non-vanishing components of $V_{A B C}$ :

$$
\begin{aligned}
& {\left[\left(x^{2}\right)^{2} y^{1}-\left(x^{1}\right)^{2} y^{2}\right]\left(y^{\alpha}\right)^{2}, \quad\left[\left(x^{3}\right)^{2} y^{1}-\eta\left(x^{1}\right)^{2} y^{3}\right]\left(y^{\alpha}\right)^{2}, \quad\left[\left(x^{4}\right)^{2} y^{1}\right]\left(y^{\alpha}\right)^{2},} \\
& {\left[\left(x^{3}\right)^{2} y^{2}-\eta\left(x^{2}\right)^{2} y^{3}\right]\left(y^{\alpha}\right)^{2},\left[\left(x^{4}\right)^{2} y^{2}\right]\left(y^{\alpha}\right)^{2},\left[\eta\left(x^{4}\right)^{2} y^{3}\right]\left(y^{\alpha}\right)^{2},} \\
& \left(x^{a}\right)^{2}\left[x^{2}\left(y^{1}\right)^{2}-x^{1}\left(y^{2}\right)^{2}\right],\left(x^{a}\right)^{2}\left[\eta x^{3}\left[\left(y^{1}\right)^{2}-x^{1}\left(y^{3}\right)^{2}\right],-\left(x^{a}\right)^{2}\left[x^{1}\left(y^{4}\right)^{2}\right],\right. \\
& \left(x^{a}\right)^{2}\left[\eta x^{3}\left(y^{2}\right)^{2}-x^{2}\left(y^{3}\right)^{2}\right],-\left(x^{a}\right)^{2}\left[x^{2}\left(y^{4}\right)^{2}\right],-\left(x^{a}\right)^{2}\left[\eta x^{3}\left(y^{4}\right)^{2}\right] .
\end{aligned}
$$

Since not all $x^{a}$ and not all $y^{\alpha}$ can vanish, for $\mathrm{d} V=0$, it must be that the quantities in the square brackets vanish. This yields the following twelve equations

$$
\begin{aligned}
\left(x^{2}\right)^{2} y^{1}-\left(x^{1}\right)^{2} y^{2} & =0, & \left(x^{4}\right)^{2} y^{1} & =0, \\
\left(x^{3}\right)^{2} y^{1}-\eta\left(x^{1}\right)^{2} y^{3} & =0, & \left(x^{4}\right)^{2} y^{2} & =0, \\
\left(x^{3}\right)^{2} y^{2}-\eta\left(x^{2}\right)^{2} y^{3} & =0, & \eta\left(x^{4}\right)^{2} y^{3} & =0, \\
x^{2}\left(y^{1}\right)^{2}-x^{1}\left(y^{2}\right)^{2} & =0, & -x^{1}\left(y^{4}\right)^{2} & =0, \\
\eta x^{3}\left(y^{1}\right)^{2}-x^{1}\left(y^{3}\right)^{2} & =0, & -x^{2}\left(y^{4}\right)^{2} & =0, \\
\eta x^{3}\left(y^{2}\right)^{2}-x^{2}\left(y^{3}\right)^{2} & =0, & -\eta x^{3}\left(y^{4}\right)^{2} & =0 .
\end{aligned}
$$


Now, Eqs. (A.2), (A.4), (A.6), (A.8), (A.10) and (A.12) enforce $x^{4}=y^{4}=0$. The only other possibility is $x^{\hat{a}}=y^{\hat{\alpha}}=0$, with $\hat{a}, \hat{\alpha} \neq 4$, which however yields $f, g, h \neq 0$. Thus, we set $x^{4}=y^{4}=0$ and proceed solving the remaining six equations patch by patch.

Let $U_{a \alpha}$ denote the patch where $x^{a}, y^{\alpha} \neq 0$. In fact, by choice of coordinates, $x^{a}=y^{\alpha}=1$ in $U_{a \alpha}$ and it remains to (a) solve Eqs. (A.1)-(A.12) for the remaining four coordinates and (b) verify that the solutions satisfy the defining equations $(2.2 \eta)$. Because of the $x^{a} \leftrightarrow y^{\alpha}$ and the $x^{1}, y^{1} \leftrightarrow x^{2}, y^{2}$ symmetries, it suffices to study the patches $U_{1 \underline{1}}, U_{12}, U_{13}$ and $U_{3 \underline{\underline{z}}}$.

We present the details for the $U_{11}$ patch only and leave the rest of the proof as an excercise for the diligent reader. In $U_{11}$, Eqs. (A.1) and (A.7) become

$$
\left(x^{2}\right)^{2}=y^{2}, \quad x^{2}=\left(y^{2}\right)^{2} .
$$

This implies that either $x^{2}=y^{2}=0$ or

$$
x^{2}=\omega^{k}, \quad y^{2}=\omega^{2 k}, \quad k=0,1,2, \omega=e^{2 i \pi / 3} .
$$

Equations (A.3) and (A.9) become

$$
\left(x^{3}\right)^{2}=\eta y^{3}, \quad \eta x^{3}=\left(y^{3}\right)^{2} .
$$

This yields that either $x^{3}=y^{3}=0$ or

$$
x^{3}=\eta \omega^{l}, \quad y^{3}=\eta \omega^{2 l}, \quad l=0,1,2 .
$$

The remaining equations are satisfied identically. We then have four possibilities

$$
\left(x^{2}, x^{3}\right)= \begin{cases}(0,0) & \Rightarrow f(x)=g(y)=h(x, y)=1, \\ \left(0, \eta \omega^{l}\right) & \Rightarrow f(x)=g(y)=h(x, y)=1+\eta^{3}, \\ \left(\omega^{k}, 0\right) & \Rightarrow f(x)=g(y)=h(x, y)=2, \\ \left(\omega^{k}, \eta \omega^{l}\right) & \Rightarrow f(x)=g(y)=h(x, y)=2+\eta^{3} .\end{cases}
$$

Equations (A.13) and the corresponding results in the remaining patches show that $d V$ and $f, g, h$ can vanish simultaneously only if $\eta^{3}=0,-1$ or -2 . (The case $\eta^{3}=0$ is not covered by the foregoing analysis, but is easy to complete along the lines above.) In other words, the space of solutions $\mathscr{M}$ of $f=g=h=0$ is smooth unless $\eta^{3}=0,-1$ or -2 .

Q.E.D.

In particular, for $\eta=1, \mathscr{M}$ is smooth and possesses an $S_{3}$ symmetry, the simultaneous permutation of the three pairs $\left(x^{1}, y^{1}\right),\left(x^{2}, y^{2}\right)$ and $\left(x^{3}, y^{3}\right)$. This case was omitted in ref. [23], but is covered in ref. [22].

A.2. The Two Smooth Quotients. Given that $\mathscr{M}$ defined in (2.2) is smooth, we now prove that the two distinct quotients $\mathscr{M}_{1}=\mathscr{M} / \varpi_{1}$ and $\mathscr{M}_{2}=\mathscr{M} / \varpi_{2}$ are also smooth.

\begin{tabular}{lcccccccc}
\hline $\mathbb{Z}_{3}$ & \multicolumn{1}{c}{ Action on } \\
& $x^{1}$ & $x^{2}$ & $x^{3}$ & $x^{4}$ & $y^{1}$ & $y^{2}$ & $y^{3}$ & $y^{4}$ \\
\hline$\varpi_{1}:$ & 1 & $\omega^{2}$ & $\omega$ & $\omega$ & 1 & $\omega$ & $\omega^{2}$ & $\omega^{2}$ \\
$\varpi_{2}:$ & 1 & $\omega^{2}$ & $\omega^{2}$ & $\omega$ & 1 & $\omega$ & $\omega$ & $\omega^{2}$ \\
\hline
\end{tabular}


The fixed point set of $\varpi_{1}$ in $\mathbb{P}_{x}^{3} \times \mathbb{P}_{y}^{3}$ can be written as

$$
\left\{(1,0,0,0),(0,1,0,0),\left(0,0, x^{3}, x^{4}\right)\right\} \times\left\{(1,0,0,0),(0,1,0,0),\left(0,0, y^{3}, y^{4}\right)\right\} .
$$

Quite manifestly, except at $\left(0,0, x^{3}, x^{4} ; 0,0, y^{3}, y^{4}\right), f(x)$ or $g(y)$ cannot vanish at these points. At $\left(0,0, x^{3}, x^{4} ; 0,0, y^{3}, y^{4}\right)$, we have that $h(x, y)=x^{3} y^{3}$ vanishes only if $x^{3}$ or $y^{3}$ does; then however

$$
\left\{\begin{array}{l}
x^{3}=0 \Rightarrow x^{4} \simeq 1 \Rightarrow f(x) \simeq 1 \neq 0 \\
y^{3}=0 \Rightarrow y^{4} \simeq 1 \Rightarrow g(y) \simeq 1 \neq 0 .
\end{array}\right.
$$

Thus, neither of the $\varpi_{1}$-fixed points satisfies the defining equations of $\mathscr{M}$, so that $\varpi_{1}$ has a free action on $\mathscr{M}$ and the quotient space $\mathscr{M}_{1} \stackrel{\text { def }}{=} \mathscr{M} / \varpi_{1}$ is smooth.

The fixed point set of $\varpi_{2}$ in $\mathbb{P}_{x}^{3} \times \mathbb{P}_{y}^{3}$ can be written as

$$
\left\{(1,0,0,0),\left(0, x^{2}, x^{3}, 0\right),(0,0,0,1)\right\} \times\left\{(1,0,0,0\},\left(0, y^{2}, y^{3}, 0\right),(0,0,0,1)\right\} .
$$

Again, except at $\left.0, x^{2}, x^{3}, 0 ; 0, y^{2}, y^{3}, 0\right), f(x)$ or $g(y)$ cannot vanish at these points. By the symmetries of the problem, it will suffice to examine the two patches in which the remaining fixed points can be parametrized as

$$
\begin{aligned}
& \stackrel{\circ}{1}_{1}=(0,1, x, 0 ; 0,1, y, 0), \\
& \stackrel{p}{2}_{2}=(0,1, x, 0 ; 0, y, 1,0) .
\end{aligned}
$$

Now, at both of these,

$$
f\left(\stackrel{\circ}{p}_{i}\right)=1+x^{3}, \quad g\left(\stackrel{\circ}{p}_{i}\right)=1+y^{3}, \quad i=1,2 .
$$

So, for $f(x)$ and $g(y)$ to vanish, we must have that

$$
\stackrel{\circ}{p}_{i}: \quad x=-\omega^{k}, \quad y=-\omega^{l}, \quad \omega=e^{2 i \pi / 3}, \quad k, l \in \mathbb{Z} .
$$

But then

$$
\begin{aligned}
& h\left(\stackrel{\circ}{1}_{1}\right)=1+\omega^{k+l} \neq 0, \\
& h\left(\stackrel{\circ}{p}_{2}\right)=\omega^{k}+\omega^{l} \neq 0,
\end{aligned}
$$

and none of the $\varpi_{2}$-fixed points lies in $\mathscr{M}$ either. Thus, the quotient space $\mathscr{M}_{2} \stackrel{\text { def }}{=} \mathscr{M} / \varpi_{2}$ is also smooth.

\section{B. Projective Preliminaries}

Here we explain the Young tableau notation and include the "tic-tac-toe" algorithm that implements the Bott-Borel-Weil (BBW) Theorem [2].

Using the fact that $\mathbb{P}^{3}=\{U(4) / U(1) \times U(3)\}$, irreducible representations of $U(1) \times U(3)$ are labeled by Young tableaux which we denote by $\left(a \mid b_{1}, b_{2}, b_{3}\right)$. Here $a$ is the $U(1)$ charge and $b_{i}$ is the number of boxes in the $i^{\text {th }}$ row (counted from the bottom), which stick out to the right (left if $b_{i}<0$ ) of the vertical "spine" of the tableau. Likewise, $\left(b_{1}, b_{2}, b_{3}, b_{4}\right)$ denote $U(4)$ Young tableaux. Note that $(1,1,1)$ is the totally antisymmetric tensor $\varepsilon^{i j k}$, which is not $U(3)$-invariant, but can be factored out; for example,

$$
(1,1,4)=(1,1,1) \otimes(0,0,3) .
$$


Since $U(3)=U(1)_{\operatorname{det}} \times S U(3)$ locally, one could say that $(1,1,4)$ and $(0,0,3)$ are equal up to the $U(1)_{\mathrm{det}}$ charge. We use this to associate tensors to the $\left(b_{1}, b_{2}, b_{3}\right)$ notation and adopt the convention that

$$
(-1,0,0) \sim \phi_{i}, \quad(0,0,1) \sim \phi^{i}, \quad i=1,2,3 .
$$

Note that the correspondence between Young tableaux and tensors is unique only if we meticulously keep track of the Levi-Civita alternating symbol. For example, $(0,1,2)$ can be represented by $\phi^{a(b c)}$, which is symmetric in $b, c$ but vanishes upon total symmetrization, but also by the traceless $\phi^{a}{ }_{b}$. Actually, their relation

$$
\phi^{a(b c)}=\varepsilon^{a b d} \phi_{d}^{c}+\varepsilon^{a c d} \phi_{d}^{b}=\varepsilon^{a e d} \delta_{e}^{(b} \delta_{f}^{c)} \phi^{f}{ }_{d}
$$

is merely the explicit form of

$$
(0,1,2)=(1,1,1) \otimes(-1,0,1) .
$$

The two tensors, therefore, differ by a unit of $U(1)_{\text {det }}$ charge. The reader unsettled by "negative boxes" in the above notation can use this factorization property to obtain, e.g.,

$$
(-1,0,0)=(-1,-1,-1) \otimes(0,1,1), \quad \phi_{i}=\varepsilon_{i j k} \phi^{[j k]} .
$$

The BBW theorem relates, in a $1-1$ fashion, a $U(1) \times U(3)$ Young tableau $\left(a \mid b_{1}, b_{2}, b_{3}\right)$ to each homogeneous vector bundle on $\mathbb{P}^{3}$. Furtheremore, $H^{q}\left(\mathbb{P}^{3},\left(a \mid b_{1}, b_{2}, b_{3}\right)\right) \neq 0$ for only one $q$ and to this cohomology group the BBW theorem assigns a $U$ (4) Young tableau $\left(c_{1}, c_{2}, c_{3}, c_{4}\right)$. For a given homogeneous bundle $\left(a \mid b_{1}, b_{2}, b_{3}\right)$, we have the following "tic-tac-toe" algorithm [2]:

1. Add the sequence $0,1,2,3$ to the respective entries in $\left(a \mid b_{1}, b_{2}, b_{3}\right)$.

2. If any two entries in the result of Step 1 are equal, all cohomology vanishes; otherwise proceed.

3. Swap the minimum number $(=q)$ of neighboring entries required to produce a strictly increasing sequence.

4. Subtract the sequence $0,1,2,3$ from the result of 3 , to obtain $\left(c_{1}, c_{2}, c_{3}, c_{4}\right)$, where $c_{\alpha} \leqq c_{\alpha+1}$, for $\alpha=1,2,3,4$.

The only non-vanishing $\left(a \mid b_{1}, b_{2}, b_{3}\right)$-valued cohomology on $\mathbb{P}^{3}$ is

$$
H^{q}\left(\mathbb{P}^{3},\left(a \mid b_{1}, b_{2}, b_{3}\right)\right)=\left(c_{1}, c_{2}, c_{3}, c_{4}\right) .
$$

For more details, see ref. $[2,16]$.

As an illustration and for orientation with the more standard notation, we list a few typical bundles on $\mathbb{P}^{3}$. We have

$$
\mathcal{O}_{\mathbb{P}^{3}}(-k)=(k \mid 0,0,0), \quad \mathscr{T}_{\mathbb{P}^{3}}=(-1 \mid 0,0,1), \quad \Omega_{\mathbb{P}^{3}}^{1}=(1 \mid-1,0,0),
$$

corresponding to $k^{\text {th }}$ order polynomials, the tangent and the cotangent bundle on $\mathbb{P}^{3}$. Other examples are easily found by the usual Kronecker product of Young tableaux and then projection to irreducible components. For example,

$$
\Omega_{\mathbb{P}^{3}}^{2}=\wedge^{2}(1 \mid-1,0,0)=[(1 \mid-1,0,0) \otimes(1 \mid-1,0,0)]_{A}=(2 \mid-1,-1,0) .
$$

Also,

$$
\Omega_{\mathbb{P}^{3}}^{1} \otimes \mathscr{T}_{\mathbb{P}^{3}}=(1 \mid-1,0,0) \otimes(-1 \mid 0,0,1)=(0 \mid-1,0,1) \oplus(0 \mid 0,0,0),
$$

which is simply a rewriting of Eq. (4.1). 


\section{Spectral Sequences}

The spectral sequence computation associated to the Koszúl complex such as (2.10) is perhaps the most uncommon of the techniques used in this article. To the best of our knowledge, available references in book form require a rather advanced understanding of algebraic geometry [1]; in fact, they do not cover this particular application. Griffiths and Harris (ref. [1], p. 438-445) give a general discussion of spectral sequences, which we shall adopt here to suit our present calculations.

Definition. A spectral sequence is a sequence $\left\{E_{i}, d_{i}\right\}(i \geqq 0)$ of bi-graded groups

$$
E_{i}=\bigoplus_{j, k \geqq 0} E_{i}^{j, k}
$$

together with differentials

$$
d_{i}: E_{i}^{j, k} \rightarrow E_{i}^{j-i, k-i-1}, \quad d_{i}^{2}=0,
$$

such that

$$
E_{i+1}=H_{d_{i}}^{\star}\left(E_{i}\right) .
$$

In practice, $d_{i} \equiv 0, i>K$ for some finite $K$, whereupon $E_{K}=E_{K+1}=\ldots$ and $E_{K}$ is called the limit of the spectral sequence, written $E_{\infty}$. The sequence of $E_{i}$ 's may be regarded as a sequence of successive approximations to $E_{\infty}$ and that is, in fact, how we use it.

For our particular application, we consider a manifold $\mathscr{M}$ embedded in $\mathbb{P}_{x}^{3} \times \mathbb{P}_{y}^{3}$ by means of a system of three $(K=3)$ defining polynomial constraints. Each defining polynomial corresponds to a line bundle, the direct sum of which we have denoted by $\mathscr{E}$. The associated Koszul complex (2.10), tensored by (the sheaf of holomorphic germs of function of) some vector bundle $\mathscr{V}$ over $\mathbb{P}_{x}^{3} \times \mathbb{P}_{y}^{3}$ is then

$$
\left.0 \longrightarrow \wedge^{3} \mathscr{E} * \otimes \mathscr{V} \stackrel{\xi}{\longrightarrow} \wedge^{2} \mathscr{E} * \otimes \mathscr{V} \stackrel{\xi}{\longrightarrow} \mathscr{E} * \otimes \stackrel{\xi}{\longrightarrow} \mathscr{V} \stackrel{\varrho}{\longrightarrow} \mathscr{V}\right|_{\mathscr{M}} \longrightarrow 0
$$

We then define

$$
E_{0}^{j, k}(\mathscr{V}) \stackrel{\text { def }}{=} H^{j}\left(\mathbb{P}_{x}^{3} \times \mathbb{P}_{y}^{3}, \mathscr{V} \otimes \wedge^{k} \mathscr{E}^{*}\right)
$$

and have the differentials, $d_{i}$ induced from the maps $\xi$. ogy

The practice of discerning the action of all $d_{i}$ 's and computing the $d_{i}$-cohomol-

$$
H_{d_{i}}^{*}\left(E_{i}(\mathscr{V})\right) \stackrel{\text { def }}{=} \underset{j, k \geqq 0}{\bigoplus}\left\{\frac{\omega \in E_{i}^{j, k}(\mathscr{V}): d_{i} \omega=0}{\omega=d_{i} \alpha, \in E_{i}^{j+i, k+i+1}(\mathscr{V})}\right\}
$$

is exemplified in Appendix D. Roughly speaking, we keep on identifying and cancelling out elements $\alpha$ and $\omega$ which are related by $\omega=d_{i} \alpha$ throughout each level $E_{i}(\mathscr{V})$ and level-by-level for $i=0,1, \ldots, K=3$.

Once $E_{\infty}(\mathscr{V})$ has been reached, we obtain the $\mathscr{V}$-valued cohomology on $\mathscr{M}$ through the combined action of maps induced from $\xi$ and $\varrho(2.12)$

$$
\left\{H^{q+k}\left(\mathbb{P}^{3} \times \mathbb{P}^{3}, \wedge^{k} \mathscr{E} * \otimes \mathscr{V}\right), \forall k: “ d_{i} \text {-closed } / d_{i} \text {-exact”, } \forall_{i}\right\} \Rightarrow H^{q}(\mathscr{M}, \mathscr{V}) .
$$


The precise mechanism and derivation of this approximation process is described in much more detail in ref. [1].

\section{Computational Details 1}

We would rather not bother the reader with all the tedium of the complete calculation of $H^{1}\left(\mathscr{M}\right.$, End $\left.\mathscr{T}_{\mathscr{M}}\right)$, but present in this and the next appendix some instructive examples.

To begin with, consider

$$
\mathscr{E}_{f} \otimes \mathscr{E}_{g} *=\left(\begin{array}{r|rrr}
-3 & 0 & 0 & 0 \\
3 & 0 & 0 & 0
\end{array}\right) .
$$

We tensor the resolution (2.13) with this and use the "tic-tac-toe" algorithm to obtain the $0^{\text {th }}$ level of the associated spectral sequence:

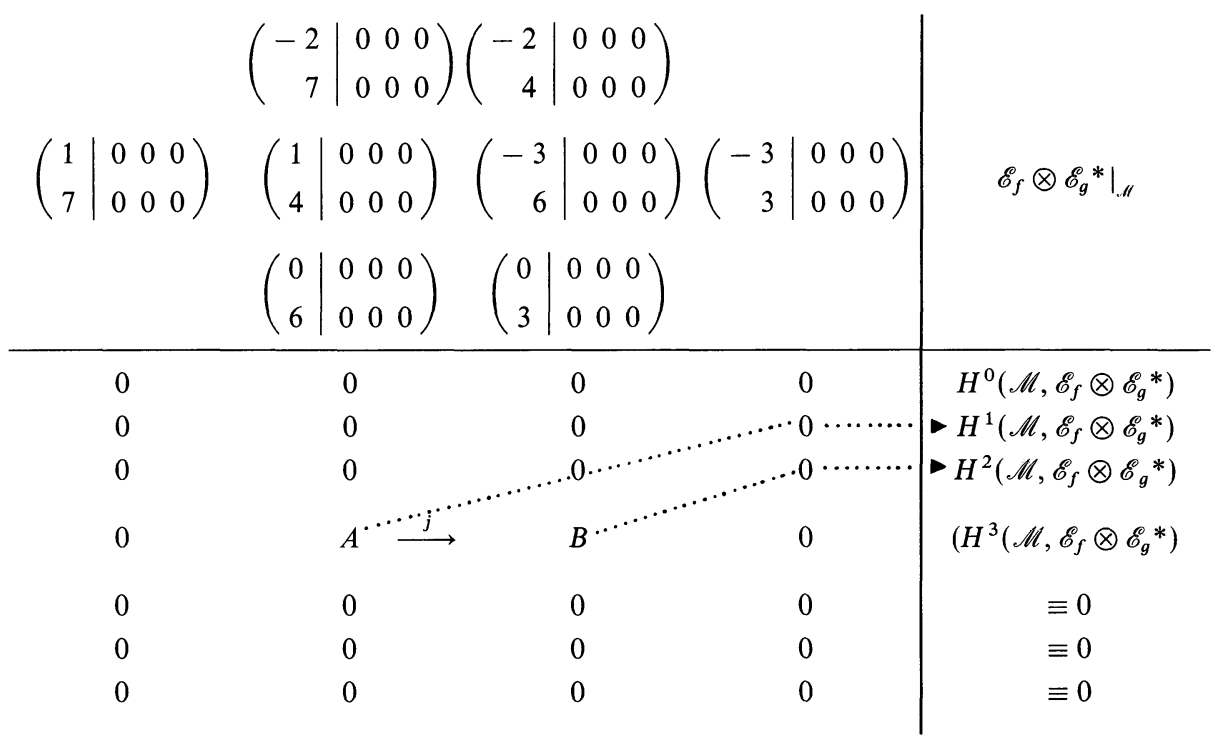

(D.1)

where $A \stackrel{j}{\rightarrow} B$ stands for

$$
\begin{aligned}
& \left(\begin{array}{rrrr}
-2 & 0 & 0 & 0 \\
1 & 1 & 1 & 4
\end{array}\right)_{20}^{10} \underset{g}{\longrightarrow}\left(\begin{array}{rrrr}
-2 & 0 & 0 & 0 \\
1 & 1 & 1 & 1
\end{array}\right)_{1}^{10} \\
& \left(\begin{array}{rrrr}
0 & 0 & 0 & 0 \\
1 & 1 & 1 & 3
\end{array}\right)_{10}^{1} \stackrel{f}{\longrightarrow}\left(\begin{array}{rrrr}
-3 & 0 & 0 & 0 \\
1 & 1 & 1 & 3
\end{array}\right)_{10}^{20}
\end{aligned}
$$

The sub- and superscripts denote the dimensions of the upper and lower factor Young tableaux, thus the product thereof is the total dimension. 
It is immediately clear that the only differential which is effective in the lower left quadrant of the above chart is the $d_{0}$ labeled explicitly by $j$. From Eq. $(2,12)$, we see that there will be no contribution to $H^{0}\left(\mathscr{M}, \mathscr{E}_{f} \otimes \mathscr{E}_{g}{ }^{*}\right)$ and $H^{3}\left(\mathscr{M}, \mathscr{E}_{f} \otimes \mathscr{E}_{g}{ }^{*}\right)$; therefore, these vanish. Now, $\operatorname{ker}(j)$ is the subspace of $A$ which is annihilated by $j$ and hence remains in the $d_{k}$-cohomology; as a subspace of $A$, it contributes to $H^{1}\left(\mathscr{M}, \mathscr{E}_{f} \otimes \mathscr{E}_{g}{ }^{*}\right)$ through Eq. (2.12). Likewise, $\operatorname{cok}(j)$ contributes to $H^{2}\left(\mathscr{M}, \mathscr{E}_{f} \otimes \mathscr{E}_{g}{ }^{*}\right)$. We therefore analyze in turn the actions of the components $h, g$ and $f$ of the mapping $j$.

$$
\begin{aligned}
\text { The } h:\left(\begin{array}{rrrr}
-2 & 0 & 0 & 0 \\
1 & 1 & 1 & 4
\end{array}\right) \rightarrow\left(\begin{array}{rrrr}
-3 & 0 & 0 & 0 \\
1 & 1 & 1 & 3
\end{array}\right) \text { mapping is realized by equating } \\
\qquad \phi_{(a b}{ }^{(\alpha \beta \gamma)} h_{c) \gamma}=\varphi_{(a b c)}{ }^{(\alpha \beta))}
\end{aligned}
$$

which is a system of 200 linear, inhomogeneous equations. The kernel of the mapping $h:\{\phi\} \rightarrow\{\varphi\}$ is, by definition, spanned by the components of $\phi_{(a b)}(\alpha \beta \gamma)$ which are annihilated by the map - regardless of the choice of $\varphi_{(a b c)}{ }^{(\alpha \beta)}$. Similarly, the cokernel of the mapping is, by definition, spanned by the components $\varphi_{(a b c)}{ }_{(\alpha \beta)}$, modulo the ideal generated by $\phi_{(a b}{ }^{(\alpha \beta \gamma)} h_{c) \gamma}$. Roughly speaking, we need to establish which of the $\phi$ 's are equated with which of the $\varphi$ 's; the remaining $\phi$ 's span $\operatorname{ker}(h)$ and the remaining $\varphi$ 's are non-trivial quotient-representatives of $\operatorname{cok}(h)$.

Note first of all that the ten components of $\phi_{(a b)}{ }^{(444)}$ do not occur in the system of equations (D.3) since $h_{c 4}=0$. These are annihilated by the map $h$ and are in its kernel. Likewise, precisely nothing is mapped to the ten components of $\varphi_{(444)}{ }^{(\alpha \beta)}$ whence they are in $\operatorname{cok}(h)$.

Since $h_{c \gamma}=\delta_{c \gamma}$ while $c, \gamma=1,2,3$ and vanishes otherwise, a suitable "splitting" of the indices is in order; indices labelled by a caret will take values only over 1,2 and 3 while the value 4 will be explicitly written. We then rewrite these 200 equations accordingly in nine groups. In the first one, all indices are restricted to take values $1,2,3$ and the assignment is

$$
\phi_{(\hat{a} \hat{b}}^{(\hat{\alpha} \hat{\beta} \hat{\gamma})} \delta_{\hat{c}) \hat{\gamma}} \mapsto \varphi_{(\hat{a} \hat{b} \hat{c})}^{(\hat{\alpha} \hat{\beta})}
$$

It is quite straightforward to see that to each of the $6 \cdot 10 \varphi$ 's a linearly independent combination of the $10 \cdot 6 \phi$ 's is assigned, so that this sector of the mapping $h$ is an isomorphism; there is no kernel or cokernel here.

Next, let $\alpha=\underline{4}$ (to distinguish from values of Latin indices, we shall underline the values of Greek indices), so that we have

$$
\phi_{(\hat{a} \hat{b}}^{(4 \hat{\beta} \hat{\gamma})} \delta_{\hat{c}) \hat{\gamma}} \mapsto \varphi_{(\hat{a} \hat{b} \hat{c})}^{(4 \hat{\beta})} .
$$

In this sector, $h$ maps a $6 \cdot 6$-component subset of $\phi$ 's to a $10 \cdot 3$-component subset of $\varphi$ 's and so cannot be $1-1$. It must annihilate at least 6 (linear combinations) of components of $\phi$. However, there is no unique set of 6 such elements and we must make some choices. With a little foresight, we eliminate 30 of the $\phi$ 's in terms of the $30 \varphi$ 's leaving

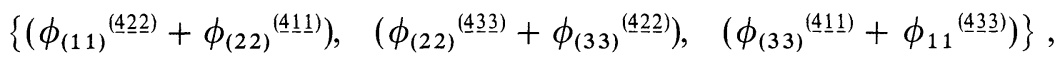

$$
\begin{aligned}
& \left\{\left(\phi_{(12)}{ }^{(43 \underline{3})}\right), \quad\left(\phi_{(13)}^{(422)}\right), \quad\left(\phi_{\left.(23)^{(41-1)}\right)}\right\}\right.
\end{aligned}
$$


to span this sector of $\operatorname{ker}(h)$; they will occur in later mappings. ${ }^{6}$

Proceeding in this fashion, let now $\alpha=\beta=\underline{4}$ :

$$
\phi_{(\hat{a} \hat{b}}^{(4-4 \hat{\gamma} \hat{\gamma})} \delta_{\hat{c}) \hat{\gamma}} \mapsto \varphi_{(\hat{a} \hat{b} \hat{c})}^{(44)} .
$$

Again the mapping $h$ must have a kernel in this sector, since (D.6) is a system of 10 equations in $6 \cdot 3$ variable $\phi$ 's. Considering the $\hat{\gamma}$ index being lowered with $\delta_{\hat{c} \hat{\gamma}}$, we rewrite the above relation as

$$
h: \phi_{(\hat{a} b \hat{b} \hat{c})^{(44)}}=\varphi_{(\hat{a} \hat{b} \hat{c})^{(44)}} .
$$

Thus

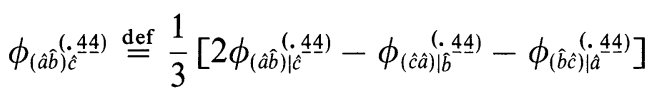

are annihilated by $h$ and span this sector of $\operatorname{ker}(h)$. We denote

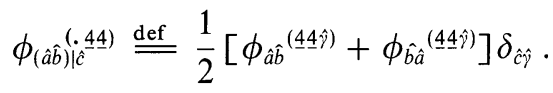

Consider next the $a=4$ case. We now have

$$
\phi_{\left(4 \hat{b}^{(\hat{\alpha} \hat{\beta} \hat{\gamma})}\right.} \delta_{\hat{c} \hat{\gamma} \hat{\gamma}}=\varphi_{(4 \hat{b} \hat{c})}^{(\hat{\alpha} \hat{\beta})},
$$

a system of 36 equations in only 30 variable components of $\left\{\phi_{4 \hat{b}}^{(\hat{\alpha} \hat{\beta} \hat{\gamma})}\right\}$. One expects that this completely determines these $\phi$ 's in terms of the $\varphi$ 's; indeed, it can be verified that these Jacobian matrix of the $\phi \rightarrow \varphi$ transformation is of maximal rank (30). Thus, the mapping has a 6-dimensional cokernel, a quotient spanned by the equivalence classes

$$
\varphi_{(4 \hat{b} \hat{c})}{ }^{(\hat{\beta} \hat{\beta})} \cong \varphi_{(4 \hat{b} \hat{c})}{ }^{(\hat{\alpha} \hat{\beta})}+\phi_{(4 \hat{b}}{ }^{(\hat{\alpha} \hat{\beta} \hat{\gamma})} \delta_{\hat{c}) \hat{\gamma}} .
$$

Clearly, there are many equivalent choices for representatives of this quotient; with the foreknowledge of the mappings that will come later, we choose the following:

$$
\begin{aligned}
& \left\{\left(\varphi_{(411)}{ }^{(22)}+\varphi_{(422)}{ }^{(111)}\right), \quad\left(\varphi_{(422)}{ }^{(333)}+\varphi_{(433)}{ }^{(22)}\right), \quad\left(\varphi_{(433)}{ }^{(11)}+\varphi_{(411)}{ }^{(\underline{3} 3)}\right)\right\}, \\
& \left.\left\{\left(\varphi_{(412)}{ }^{(\underline{3})}\right), \quad\left(\varphi_{(423)}{ }^{(11)}\right), \quad \varphi_{(431)}{ }^{(22)}\right)\right\}
\end{aligned}
$$

Collecting all such contributions, for various other arrangements of the ranges of the five free indices in (D.3), we find that the kernel of the map $h$ is spanned by

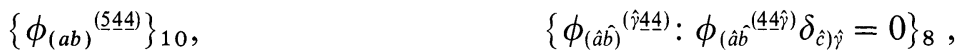

$$
\begin{aligned}
& \left\{\left(\phi_{(4 \hat{b})}^{(44 \hat{\gamma})} \delta^{\hat{b} \hat{\beta}}-\phi_{(4 \hat{b})}^{(44 \hat{\beta})} \delta^{\hat{b} \hat{\gamma}}\right)=0\right\}_{3}, \quad\left\{\phi_{(\hat{a} \hat{b})}^{(\hat{\gamma} \hat{\delta} \hat{d})}: \phi_{(\hat{a} \hat{b}}^{(4 \hat{\gamma} \hat{\delta})} \delta_{\hat{c}) \hat{\gamma}}=0\right\}_{6}
\end{aligned}
$$

\footnotetext{
${ }^{6}$ The situation here is a little like solving a simple relation $x+y=a$, knowing that later another relation, $x=b$, will be encountered. With this foresight, we use the first relation to express $y=y(x, a)$ and leave $x$ free (for the later relation to fix it). More properly, when the later relation is encountered, we should backup and combine the relations into a system and then solve this complete system. However, in practice, we would have to back up several times and the resulting analysis grows beyond reasonable means of presentation in published form. It is however not hard to check our calculations in this way and verify that no loss of generality in the end result was incurred through our somewhat sinuous procedure
} 
while

$$
\begin{aligned}
& \left\{\varphi_{(444)}^{(\alpha \beta)}\right\}_{10}, \\
& \left\{\varphi_{(44 \hat{c})}^{(\hat{\alpha} \hat{\beta})} \cong \varphi_{(44 \hat{c})}^{(\hat{\alpha} \hat{\beta})}+\phi_{(44)}{ }^{(\hat{\alpha} \hat{\beta} \hat{\gamma})} \delta_{\hat{c} \hat{\gamma}}\right\}_{8}, \\
& \left\{\left(\varphi_{(44 \hat{b})}^{(4 \hat{\gamma})} \delta_{\hat{c} \hat{\gamma}}-\varphi_{(44 \hat{c})}{ }^{(4 \hat{\gamma})} \delta_{\hat{b \hat{\gamma}}}\right)\right\}_{3}, \\
& \left\{\varphi_{(4 \hat{b} \hat{c})}^{(\hat{\alpha} \hat{\beta})} \cong \varphi_{(4 \hat{b \hat{c}})}{ }^{(\hat{\alpha} \hat{\beta})}+\phi_{(4 \hat{b}}^{(\hat{\alpha} \hat{\beta} \hat{\gamma})} \delta_{\hat{c}) \hat{\gamma}}\right\}_{6}
\end{aligned}
$$

span the cokernel.

Next in discussing the combined mapping in (D.2), we consider the $g:\left(\begin{array}{rrrr}-2 & 0 & 0 & 0 \\ 1 & 1 & 1 & 4\end{array}\right) \rightarrow\left(\begin{array}{rrrr}-2 & 0 & 0 & 0 \\ 1 & 1 & 1 & 1\end{array}\right)$ map, realized by setting

$$
\phi_{(a b)}{ }^{(\alpha \beta \gamma)} g_{\alpha \beta \gamma}=\kappa_{(a b)} \text {. }
$$

Because of the symmetric choice (2.2), only the $\phi$ 's with $\alpha=\beta=\gamma$ can be mapped by $g$ acting as above. Among these, the $\phi_{(a b)}^{(\hat{\alpha} \hat{\alpha} \hat{\alpha})}$ 's have occurred in the domain of $h$ in (D.3), where it was invertible (no kernels in those sectors). Thus, we denote loosely $\phi_{(a b)}^{(\hat{\alpha} \alpha \hat{\alpha})}=h^{-1}(\varphi)$ and substitute in (D.9) to obtain

$$
g: \phi_{(a b)}{ }^{(444)}=\kappa_{(a b)}-h^{-1}(\varphi),
$$

the right-hand side of which vanishes only for special choices of the $\varphi$ 's. Thus, no component of $\phi_{(a b)}{ }^{(444)}$ is annihilated by $g$ and $\operatorname{ker}(g)=0$.

Since $\operatorname{ker}(g)=0, g$ must be 1-1 and since furthermore the dimensions of the spaces being mapped, $\left\{\phi_{(a b)}{ }^{(444)}\right\}_{10}$ and $\left\{\kappa_{(a b)}\right\}_{10}$, are the same $-g$ must be an isomorphism. More explicitly, consider the equivalence classes

$$
\kappa_{(a b)} \cong \kappa_{(a b)}+\phi_{(a b)}{ }^{(444)}+h^{-1}(\varphi),
$$

which span $\operatorname{cok}(g)$. Quite clearly, the ideal generated by the $\phi_{(a b)}{ }^{(444)}$ is sufficient to render all $\kappa$ 's equivalent to zero: using these $\phi$ 's, we can gauge away all $\kappa$ 's.

The $8+3+6$ elements from (D.7) are however annihilated by the map $g$ (D.9) and so they span the kernel of the combined mapping (D.2). Since there are no other maps, these contributions survive into the final level of the spectral sequence (D.1) and filter directly into $H^{1}\left(\mathscr{M}, \mathscr{E}_{f} \otimes \mathscr{E}_{g}{ }^{*}\right)$. Likewise, only the $8+3+6$ components from (D.8) remain to $\operatorname{span} H^{2}\left(\mathscr{M}, \mathscr{E}_{f} \otimes \mathscr{E}_{g}{ }^{*}\right)$.

An analogous procedure is then exercised with the resolution (2.10) tensored with the remaining 17 irreducible bundles found in (4.5).

\section{E. Computational Details 2}

This appendix contains another sample computation along the lines described in Sects. 3 and 4, aiming to illustrate the straightforwardness of our calculations, albeit being technically laborous.

Having determined the cohomology groups on $\mathscr{M}$ valued in the various bundles (4.5) as described in the previous appendix, we can now proceed to compute $H^{q}\left(\mathscr{M}\right.$, End $\left.\mathscr{T}_{\mathscr{M}}\right)$ in two steps: first, determining all the relative cohomology for the row exact sequence in (4.4) from the column exact sequences and then $H^{q}\left(\mathscr{M}\right.$, End $\left.\mathscr{T}_{\mathscr{M}}\right)$ from that row exact sequence.

Our sample computation will concern a component of the right-most column exact sequence in (4.4), involving the $\mathscr{E}_{f}$ component of $\mathscr{E}$ :

$$
0 \rightarrow \mathscr{E}_{f} \otimes \mathscr{E}^{*} \rightarrow \mathscr{E}_{f} \otimes \mathscr{T}_{\mathscr{W}}^{*} \rightarrow \mathscr{E}_{f} \otimes \mathscr{T}_{\mathscr{M}}^{*} \rightarrow 0
$$


This was clearly obtained from the sequence (2.5), by tensoring with $\mathscr{E}_{f}$. As usual, the short exact sequence (E.1) induces the long exact sequence of cohomology groups

$$
\begin{aligned}
0 \rightarrow \ldots & \rightarrow H^{q}\left(\mathscr{M}, \mathscr{E}_{f} \otimes \mathscr{E}^{*}\right) \rightarrow H^{q}\left(\mathscr{M}, \mathscr{E}_{f} \otimes \mathscr{T}_{\mathscr{W}}^{*}\right) \rightarrow H^{q}\left(\mathscr{M}, \mathscr{E}_{f} \otimes \mathscr{T}_{\mathscr{M}}^{*}\right) \\
& \rightarrow H^{q+1}\left(\mathscr{M}, \mathscr{E}_{f} \otimes \mathscr{E}^{*}\right) \rightarrow \ldots \rightarrow 0 .
\end{aligned}
$$

Using the results obtained thus far, we have an explicit representation for all $H^{q}\left(\mathscr{M}, \mathscr{E}_{f} \otimes \mathscr{T}_{\mathscr{W}}^{*}\right)$ and $H^{q}\left(\mathscr{M}, \mathscr{E}_{f} \otimes \mathscr{E}^{*}\right)$. We now discuss this row by row, for $q=0,1,2,3$.

For $q=0$, we have

$$
\begin{aligned}
0 & \rightarrow\left(\begin{array}{llll}
0 & 0 & 0 & 0 \\
0 & 0 & 0 & 0
\end{array}\right)_{1}^{1} \stackrel{j_{0}}{\longrightarrow}\left[\left(\begin{array}{llll}
0 & 0 & 0 & 0 \\
0 & 0 & 0 & 0
\end{array}\right)_{1}^{1}+\left(\begin{array}{rrrr}
-2 & -1 & 0 & 0 \\
0 & 0 & 0 & 0
\end{array}\right)_{1}^{20}\right] \\
& \stackrel{i_{0}}{\longrightarrow} H^{0}\left(\mathscr{M}, \mathscr{E}_{f} \otimes \mathscr{T}_{\mathscr{M}}^{*}\right) \longrightarrow \ldots
\end{aligned}
$$

The map $j_{0}$ here is clearly the identity and cancels the two 1-dimensional Young stableaux, so that $i_{0}$ takes $\left(\begin{array}{rrrr}-2 & -1 & 0 & 0 \\ 0 & 0 & 0 & 0\end{array}\right)_{1}^{20}$ over into $H^{0}\left(\mathscr{M}, \mathscr{E}_{f} \otimes \mathscr{T}_{\mathscr{M}}^{*}\right)$. Tensors corresponding to this Young tableaux are easily seen to be of the type $\phi_{a(b c)}$ (which vanishes upon total symmetrization) and will give rise to the contributions also found by deformation theory (4.3).

The situation in rows $q=2,3$ is particularly simple, since $H^{q}\left(\mathscr{M}_{,} \mathscr{E}_{f} \otimes \mathscr{T}_{\mathscr{W}}^{*}\right)$ vanishes there. The maps $i_{2}$ and $i_{3}$ thus have nothing to map into $H^{2}\left(\mathscr{M}, \mathscr{E}_{f} \otimes \mathscr{T}_{\mathscr{M}}^{*}\right)$ and $H^{3}\left(\mathscr{M}, \mathscr{E}_{f} \otimes \mathscr{T}_{\mathscr{M}}^{*}\right)$, respectively. Therefore, the only contribution to the first one comes from $H^{3}\left(\mathscr{M}, \mathscr{E}_{f} \otimes \mathscr{E}^{*}\right)$, through the dimensionchanging map $\delta_{2}$, and equals $\left(\begin{array}{llll}1 & 1 & 1 & 1 \\ 1 & 1 & 1 & 1\end{array}\right)_{1}^{1}$.

With this established, we remain with

$$
\left.\begin{array}{c}
0 \longrightarrow\left(\begin{array}{rrrr}
-2 & 0 & 0 & 0 \\
0 & 0 & 0 & 0
\end{array}\right)_{1}^{20} \longrightarrow H^{0}\left(\mathscr{M}, \mathscr{E}_{f} \otimes \mathscr{T}_{\mathscr{M}}^{*}\right) \\
\stackrel{\delta_{0}}{\longrightarrow}\left[\begin{array}{l}
\mathbf{A} \\
\mathbf{B}
\end{array}\right] \underset{h}{\longrightarrow}\left[\begin{array}{rrrr}
-1 & -1 & 0 & 0 \\
1 & 1 & 1 & 1
\end{array}\right)_{1}^{6} \\
\mathbf{C}
\end{array}\right]
$$

where we need to discern the precise action of the combined map in the middle. Contributions to $H^{0}\left(\mathscr{M}, \mathscr{E}_{f} \otimes \mathscr{T}_{\mathscr{M}}^{*}\right)$ will then be the leftmost Young tableau and the kernel of the combined mapping, while $H^{1}\left(\mathscr{M}_{,} \mathscr{E}_{f} \otimes \mathscr{T}_{\mathscr{M}}^{*}\right)$ will be built up from 
the cokernel of the combined mapping and the rightmost contribution, $\mathbf{D}$ and $\mathbf{E}$. The four labels above denote:

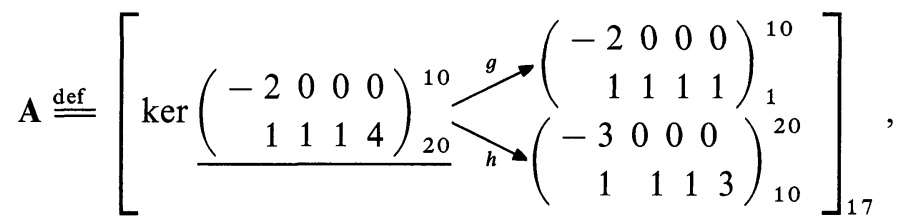

$$
\begin{aligned}
& \mathbf{B} \stackrel{\text { def }}{=}\left[\operatorname{ker}\left(\begin{array}{rrrr}
-1 & 0 & 0 & 0 \\
1 & 1 & 1 & 2
\end{array}\right)_{4}^{4} \stackrel{h}{\longrightarrow}\left(\begin{array}{rrrr}
-2 & 0 & 0 & 0 \\
1 & 1 & 1 & 1
\end{array}\right)_{1}^{10}\right]_{7}, \\
& \mathbf{C} \stackrel{\text { def }}{=}\left[\operatorname{ker}\left(\begin{array}{rrrr}
-2 & 0 & 0 & 0 \\
0 & 1 & 1 & 2
\end{array}\right)_{15}^{10} \stackrel{h}{\longrightarrow}\left(\begin{array}{rrrr}
-3 & 0 & 0 & 0 \\
1 & 1 & 1 & 1
\end{array}\right)_{1}^{20}\right]_{74}, \\
& +\left[\operatorname{cok}\left(\begin{array}{llll}
0 & 0 & 0 & 0 \\
0 & 0 & 0 & 0
\end{array}\right)_{1}^{1} \stackrel{f}{\longrightarrow}\left(\begin{array}{rrrr}
-3 & 0 & 0 & 0 \\
0 & 0 & 0 & 0
\end{array}\right)_{1}^{20}\right]_{19}
\end{aligned}
$$

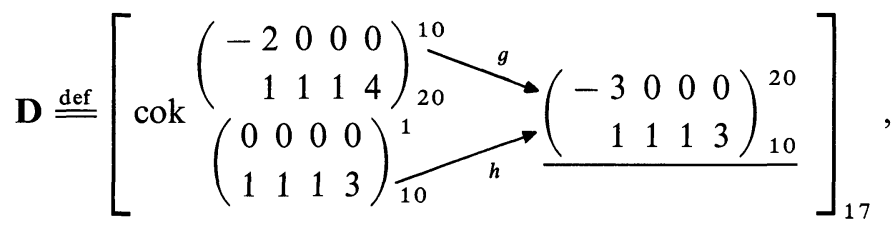

$$
\begin{aligned}
& \mathbf{E} \stackrel{\text { def }}{=}\left[\operatorname{cok}\left(\begin{array}{rrrr}
-1 & 0 & 0 & 0 \\
1 & 1 & 1 & 2
\end{array}\right)_{4}^{4} \stackrel{h}{\longrightarrow}\left(\begin{array}{rrrr}
-2 & 0 & 0 & 0 \\
1 & 1 & 1 & 1
\end{array}\right)_{1}^{10}\right]_{1},
\end{aligned}
$$

where we have underlined the Young tableaux which are the source or the target of the maps.

We now turn to study the combined mapping and begin with the map $g$. The assignments are determined by the Koszul complex and correspond in tensorial notation to

$$
g_{\alpha \hat{\beta} \hat{\gamma}} \phi_{(a b)}^{\hat{\beta} \hat{\gamma} \delta}-\frac{1}{4} \delta_{\alpha}^{\delta} g_{\hat{\beta} \hat{\gamma} \kappa} \phi_{(a b)}^{\hat{\beta} \hat{\gamma} \kappa} \rightarrow \vartheta_{(a b) \alpha}^{\delta} \in\left(\begin{array}{rrrr}
-2 & 0 & 0 & 0 \\
0 & 1 & 1 & 2
\end{array}\right)_{15}^{10},
$$

where $\delta_{\alpha}^{\delta}$ is the $4 \times 4 \mathrm{Kronecker}$ delta-symbol. The subtraction on the left-hand side ensures tracelessness, as dictated by the Young tableau represented by the $\vartheta$ 's.

It will be useful to break up the ranges of the free indices into three groups as in the list (D.7) and we now discuss these in turn.

a) $g_{\hat{\gamma} \hat{\gamma} \hat{\gamma}} \phi_{(\hat{a} \hat{b})}^{\hat{\gamma} \hat{\gamma} \underline{4}} \mapsto \vartheta_{(\hat{a} \hat{b}) \hat{\gamma} \underline{4}}$. Using the various constraints on the components of $\phi$ and $\vartheta$ from before,

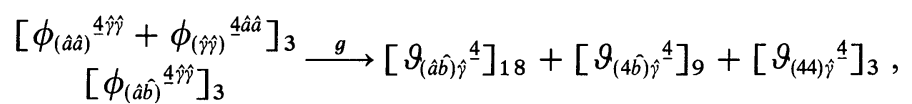


where $\hat{a}, \hat{b}$ and $\hat{\gamma}$ are all different. Clearly, the map has its image only in the first summand on the right-hand side, and in fact maps out $\frac{1}{2}\left(\vartheta_{(\hat{a} \hat{a}) \hat{\vartheta}}{ }^{4}+\vartheta_{(\hat{\hat{\gamma}}) \hat{a}^{\hat{a}}}\right)$ and $\vartheta_{(\hat{a} \hat{b}) \hat{y}}^{4}$.

b) $g_{444} \phi_{(\hat{a} \hat{b})}^{44 \hat{\gamma}} \mapsto \vartheta_{(\hat{a} \bar{b}) \underline{\hat{\gamma}}}$. Again, by various constraints on $\phi$ and $\vartheta$ from before, in this range of indices,

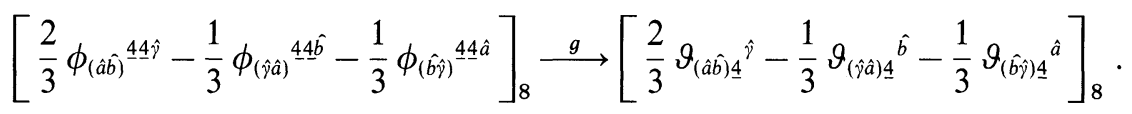

This map is clearly an isomorphism and has no kernel or cokernel among the displayed components.

c) $g_{444} \phi_{(4 \hat{b})}{ }^{44 \hat{\gamma}} \mapsto \vartheta_{(4 \hat{b}) 4} \hat{\hat{p}}$. With the various constraints on $\phi$ and $\vartheta$ from before, we now have

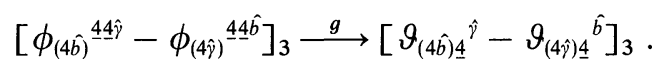

Again, the map is clearly an isomorphism and has no kernel or cokernel among the displayed components.

This has completely annihilated $\mathbf{A}$ and has cancelled 17 components of $\mathbf{C}$.

Consider now the map $\mathbf{B} \stackrel{h}{\longrightarrow}\left(\begin{array}{rrrr}-1 & -1 & 0 & 0 \\ 1 & 1 & 1 & 1\end{array}\right)_{1}^{6}$. With the 7 components of B, we have

$$
\left\{\left(\phi_{\hat{a}}^{\hat{\alpha}}-\phi_{\hat{\alpha}}^{\hat{a}}\right), \phi_{\hat{a}}{ }^{4}, \phi_{4}{ }^{4}\right\} \stackrel{h}{\longrightarrow}\left\{\vartheta_{[\hat{a} \hat{b}]}, \vartheta_{[\hat{a} 4]}\right\} .
$$

The only possible assignment here is $\phi_{[\hat{a}}^{\hat{\gamma}} h_{\hat{b}] \hat{\gamma}} \mapsto \vartheta_{[\hat{a} \hat{b}]}$, leaving $\left\{\vartheta_{[\hat{a} 4]}\right\}_{3}$ in the cokernel and $\left\{\phi_{a}{ }^{4}\right\}_{4}$ in the kernel.

Finally, we consider the map $\mathbf{B} \stackrel{h}{\longrightarrow}$ C, substituting what has been left of these. The involved components are:

$$
\phi_{a} \stackrel{4}{\longrightarrow} \vartheta_{(\hat{a} \hat{b}) \mid b^{4}} \oplus \vartheta_{(4 \hat{b}) \mid \hat{\gamma}^{4}} .
$$

The three components $\phi_{\hat{a}^{4}}$ are clearly mapped out of the first $\vartheta$, while $\phi_{4}{ }^{4}$ gets mapped out of the second $\vartheta$. This leaves no kernel in this sector and the cokernel is described by the "mod- $h\left(\phi_{a}{ }^{4}\right)$ " equivalence classes of the two respective $\vartheta$ 's.

Collecting all these results, we now have $H^{q}\left(\mathscr{M}_{\mathscr{E}_{f}} \otimes \mathscr{T}_{\mathscr{M}}^{*}\right)$. For $q=0$, this cohomology follows from Eq. (E.3) and is spanned by the 20 components of $\phi_{(a b) c}$, corresponding to $\left(\begin{array}{rrrr}-2 & -1 & 0 & 0 \\ 0 & 0 & 0 & 0\end{array}\right)_{1}^{20}$.

For $q=1$, we obtain a large collection of different contributions:

$$
\begin{aligned}
& \left\{\phi_{(\hat{a} \hat{b}) \mid b^{4}}{ }^{4} \lambda_{(\hat{a}} h_{\hat{b}) \hat{b}}\right\}_{9-3}=6, \quad\left\{\phi_{(4 \hat{b}) \mid \hat{\gamma}}{ }^{4} / \lambda_{4}{ }^{4} h_{\hat{b} \hat{\gamma}}\right\}_{9-1=8}, \quad\left\{\phi_{(44) \mid b^{4}}\right\}_{3}, \\
& \left\{\phi_{(\hat{a} \hat{a}) \mid \hat{\gamma}^{4}}-\phi_{(\hat{\gamma} \hat{)}) \mid a^{4}}\right\}_{3}, \quad\left\{\phi_{(4 \hat{b}) \mid \delta^{\hat{\gamma}}}-\phi_{(4 \hat{y}) \mid \delta}\right\}_{9}^{\hat{b}}, \quad\left\{\phi_{(\hat{a} \hat{b}) \cdot \hat{\gamma}}\right\}_{24}, \\
& \left\{\phi_{[\hat{a} 4]}\right\}_{3}, \quad\left\{\phi_{(a b c)} / \lambda f_{a b c}\right\}_{19}, \quad\left\{\phi_{(4 \hat{b} \hat{b})}^{(\hat{\alpha} \hat{\alpha})+} \phi_{(4 \hat{\alpha} \hat{\alpha})}^{(\hat{b} \hat{b})}, \hat{\alpha} \neq \hat{\beta}\right\}_{3},
\end{aligned}
$$

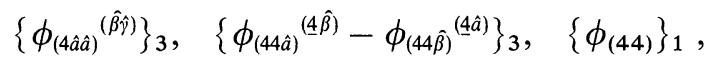

$$
\begin{aligned}
& \left\{\frac{2}{3} \phi_{(44 \hat{a})}{ }^{(\hat{\beta} \hat{\gamma})}-\frac{1}{3} \phi_{(44 \hat{\gamma})}^{(\hat{a} \hat{\beta})}-\frac{1}{3} \phi_{(44 \hat{\beta})}^{(\hat{\gamma} \hat{a})}\right\}_{8} .
\end{aligned}
$$


Finally, $H^{2}\left(\mathscr{M}, \mathscr{E}_{f} \otimes \mathscr{T}_{\mathscr{M}}^{*}\right)$ equals $\left(\begin{array}{llll}1 & 1 & 1 & 1 \\ 1 & 1 & 1 & 1\end{array}\right)$ and is represented by a constant multiple of $\varepsilon^{1234} \varepsilon^{123}{ }_{4}$ and $H^{3}\left(\mathscr{M}, \mathscr{E}_{f} \otimes \mathscr{T}_{\mathscr{M}}^{*}\right)$ vanishes.

\section{References}

1. Hartshorne, R.: Algebraic geometry. Berlin, Heidelberg, New York: Springer 1977; Griffiths, P., Harris, J.: Principles of algebraic geometry. New York: John Wiley 1978; Wells, R.O.: Differential analysis on complex manifolds. Berlin, Heidelberg, New York: Springer 1980; Bott, R., Tu, L.: Differential forms and algebraic topology. Berlin, Heidelberg, New York: Springer 1982; Matsumura, H.: (translated by M. Reid) Commutative ring theory. Cambridge: Cambridge University Press 1986

2. Eastwood, M.G.: Math. Proc. Camb. Phil. Soc. 97, 165 (1985)

3. Green, P., Hübsch, T.: Commun. Math. Phys. 113, 505 (1987)

4. Green, P.S., Hübsch, T., Lütken, C.A.: Class. Quant. Grav. 6, 105 (1989)

5. Berglund, P., Hübsch, T.: Phys. Lett. B260, 32-38 (1991)

6. Greene, B.: Phys. Rev. D40, 1145 (1989)

7. Yau, S.T.: In: Proc. of Symposium on Anomalies, Geometry, Topology, pp. 395, especially the Appendix, written by Tian, G., Yau, S.-T. pp. 402-405, Bardeen, W.A., White, A.R.: (eds.) Singapore: World Scientific 1985

8. Candelas, P., Horowitz, G., Strominger, A., Witten, E.: Nucl. Phys. B258, 46 (1985)

9. Greene, B.R., Kirklin, K.H., Miron, P.J., Ross, G.G.: Nucl. Phys. B278, 667 (1986)

10. Green, M.B., Schwarz, J.H., Witten, E.: Superstring Theory II. Cambridge: Cambridge University Press 1987

11. Candelas, P.: Nucl. Phys. B298, 458 (1988)

12. Distler, J., Greene, B., Kirklin, K., Miron, P.: Phys. Lett. 195B, 41 (1987)

13. Green, P., Hübsch, T.: Class Quant. Grav. 6, 311 (1989)

14. Hübsch, T.: Commun. Math. Phys. 108, 291 (1987)

15. Distler, J., Greene, B., Kirklin, K., Miron, P.: Commun. Math. Phys. 112, 177 (1989)

16. Eastwood, M.G., Hübsch, T.: Commun. Math. Phys. 132, 383 (1990)

17. Berglund, P., Hübsch, T., Parkes, L.: Mod. Phys. Lett. A5, 1485 (1990)

18. Dine, M., Seiberg, N., Wen, X.-G., Witten, E.: Nucl. Phys. B278, 769 (1986) ibid. B289, 319 (1987)

19. Witten, E.: Nucl. Phys. B268, 79 (1986)

20. Ross, G.G.: Phys. Lett. 221B, 315 (1988)

21. Candelas, P., Kalara, S.: Nucl. Phys. B298, 357 (1988)

22. Lazarides, G., Shafi, Q.: J. Math. Phys 36, 711 (1989)

23. Ganoulis, N., Lazarides, G., Shafi, Q.: Nucl. Phys. B323, 374 (1989)

24. Okonek, C., Schneider, M., Spindler, H.: Vector bundles on complex projective spaces. Basel: Birkhäuser 1980; Uhlenbeck, K., Yau, S.-T.: Commun. Pure and Appl. Math. 39, S257-S293 (1986)

25. Bento, M.C., Hall, L., Ross, G.G.: Nucl. Phys. B292, 400 (1987)

26. Wang, X.-M.: Nucl. Phys. B343, 129 (1990)

27. Wall, C.T.C.: Invent. Math. 1, 355 (1966)

28. Dixon, L.: In: Superstrings, Unified Theories and Cosmology 1987, pp. 67-127 Furlan, G. et al. (eds.) Singapore: World Scientific 1988

29. Green, P., Hübsch, T.: Commun. Math. Phys. 109, 99 (1987) 